TRANSACTIONS OF THE

AMERICAN MATHEMATICAL SOCIETY

Volume 349, Number 1, January 1997, Pages 331-372

S 0002-9947(97)01729-7

\title{
ON THE COMPLETE INTEGRABILITY OF SOME LAX EQUATIONS ON A PERIODIC LATTICE
}

\author{
LUEN-CHAU LI
}

\begin{abstract}
We consider some Lax equations on a periodic lattice with $N=2$ sites under which the monodromy matrix evolves according to the Toda flows. To establish their integrability (in the sense of Liouville) on generic symplectic leaves of the underlying Poisson structure, we construct the action-angle variables explicitly. The action variables are invariants of certain group actions. In particular, one collection of these invariants is associated with a spectral curve and the linearization of the associated Hamilton equations involves interesting new feature. We also prove the injectivity of the linearization map into real variables and solve the Hamilton equations generated by the invariants via factorization problems.
\end{abstract}

\section{InTRODUCTION}

Over the past decade there has been a great deal of activity in the solution of nonlinear evolution equations in $1+1$ dimensions by the Riemann problem method (see $[\mathrm{FT}]$ and references therein). As is well-known, at the basis of all these works is the representation of the equations as a condition of zero curvature, i.e.

$$
\frac{\partial U}{\partial t}-\frac{\partial V}{\partial x}+[U, V]=0
$$

Here, $U$ and $V$ are matrix-valued functions parametrized by the classical fields and $[\cdot, \cdot]$ is the standard commutator. For periodic lattice models, where the (discretized) spatial variable $n$ now takes values in $\mathbf{Z}_{N}=\mathbf{Z} / N \mathbf{Z}$, there is a natural analog of (1.1). These are the so-called Lax equations on a lattice (or lattice Lax system) $[\mathrm{AL}],[\mathrm{FT}]$, and they have the general form

$$
\dot{g}_{k}=v_{k+1} g_{k}-g_{k} v_{k}, \quad k \in \mathbf{Z}_{N} .
$$

The matrices $g_{k}$ above are invertible and define parallel transport from site $k$ of the lattice to site $k+1[\mathrm{FT}]$. As can be easily verified, the monodromy matrix $T(g)=g_{N} \ldots g_{1}, g=\left(g_{1}, \ldots, g_{N}\right)$, undergoes an isospectral deformation

$$
\dot{T}(g)=\left[v_{1}, T(g)\right],
$$

and hence the eigenvalues of $T(\mathrm{~g})$ provide a collection of conserved quantities for (1.2). In this paper, we shall study some particular Lax systems on a lattice with $N=2$ sites which are related to the Toda flows [DLT]. Without loss of generality,

Received by the editors May 16, 1994 and, in revised form, October 6, 1995.

1991 Mathematics Subject Classification. Primary 58F07; Secondary 58F05, 65F15.

(C)1997 American Mathematical Society 
we may assume $g_{1}, g_{2}$ lie in the identity component $G$ of the group of real, invertible $n \times n$ matrices. It is well-known that $G$ admits the global decomposition

$$
G=K \cdot L,
$$

where $K$ and $L$ are the subgroups of orthogonal matrices and lower triangular matrices respectively. On the Lie algebra level, we have

$$
\mathfrak{g}=\mathfrak{k} \oplus \mathfrak{l} .
$$

Therefore, if $\Pi_{\mathfrak{k}}$ and $\Pi_{\mathfrak{l}}$ are the projection operators corresponding to the direct sum decomposition, then

$$
R=\Pi_{\mathfrak{k}}-\Pi_{\mathfrak{l}}
$$

is a classical $r$-matrix [STS1]. As a final ingredient for the equations, we take a central function $\varphi$ on $G$, i.e. $\varphi\left(x y x^{-1}\right)=\varphi(y), x, y \in G$, and let

$$
H_{\varphi}\left(g_{1}, g_{2}\right)=\varphi(T(g)), T(g)=g_{2} g_{1}, g=\left(g_{1}, g_{2}\right) .
$$

The Lax equations corresponding to $R$ and $\varphi$ on a periodic lattice with $N=2$ sites take the form

$$
\begin{aligned}
& \dot{g}_{1}=\frac{1}{2} R\left(D_{1} H_{\varphi}(g)\right) \cdot g_{1}-\frac{1}{2} g_{1} \cdot R\left(D_{2} H_{\varphi}(g)\right), \\
& \dot{g}_{2}=\frac{1}{2} R\left(D_{2} H_{\varphi}(g)\right) \cdot g_{2}-\frac{1}{2} g_{2} \cdot R\left(D_{1} H_{\varphi}(g)\right),
\end{aligned}
$$

where $D_{i} H_{\varphi}(g)=g_{i}\left(\nabla_{i} H_{\varphi}(g)\right)^{T}, i=1,2$, are the left gradients (here $\nabla_{i} H_{\varphi}(g)$ is the $n \times n$ matrix whose $(j, k)$ entry is $\left.\partial H_{\varphi}(g) / \partial\left(g_{i}\right)_{j k}\right)$. For readers familiar with the work of M. Semenov-Tian-Shansky [STS1], [STS2], let us remark that equations of this sort were introduced by him in the case of skew-symmetric $r$-matrices; however, $R$ as defined in (1.6) above is not skew-symmetric and the Hamiltonian formalism for such equations was extended by the author and his collaborator in [LP]. As particular examples of (1.8), we have the equations

$$
\begin{aligned}
& \dot{g}_{1}=\frac{1}{2} R\left(\left(g_{1} g_{2}\right)^{k}\right) \cdot g_{1}-\frac{1}{2} g_{1} \cdot R\left(\left(g_{2} g_{1}\right)^{k}\right), \\
& \dot{g}_{2}=\frac{1}{2} R\left(\left(g_{2} g_{1}\right)^{k}\right) \cdot g_{2}-\frac{1}{2} g_{2} \cdot R\left(\left(g_{1} g_{2}\right)^{k}\right), \quad k=1,2, \ldots,
\end{aligned}
$$

under which the monodromy matrix $T(g)$ evolves according to the Toda flows [DLT], i.e.

$$
\dot{T}(g)=\frac{1}{2}\left[R\left((T(g))^{k}\right), T(g)\right], \quad k=1,2, \ldots
$$

Thus (1.9) is in some sense a reduction of the Toda flows, which we know are completely integrable on generic symplectic leaves of an associated Poisson structure on the group $G[\mathrm{LP}]$ (see, however [DLT] for a detailed discussion of the integrals). In this paper, we shall show that equations of the form (1.8) (and in particular (1.9)) are also completely integrable on generic symplectic leaves of the associated Poisson structure on $G^{2}$. Apart from the intrinsic interest from the point of view mentioned above (see also the description of results below), there are connections with algorithms in numerical linear algebra and the generalized KdV hierarchies of Drinfeld and Sokolov [DS]. For example, if we take $k=1$ in (1.9), the equations for $g_{1}$ and $g_{2}^{-1}$ are exactly the ones considered in [C]. On the other hand, it is clear that the equations in (1.8) extend naturally to complex matrices, and if we take $\varphi(x)=-\frac{1}{2} \operatorname{tr}(\log x)^{2}$, the resulting flow on $\left(g_{1}, g_{2}^{-1}\right)$ is nothing but a continuous 
time interpolation of the QZ algorithm [MS] to compute generalized eigenvalues of the pair $\left(g_{1}, g_{2}^{-1}\right)$. Of course, we can also interpret (1.9) with the above choice of $\varphi$ as an algorithm to compute the eigenvalues of the product $g_{2} g_{1}$ (or $g_{1} g_{2}$ ). We now explain the connection with partial differential equations. Consider, say, the Gelfand-Dikii equation [GD]

$$
\dot{L}=\left[\left(L^{k / n}\right)_{+}, L\right],
$$

where

$$
L=D^{n}+\sum_{i=1}^{n-1} u_{i} D^{i}, \quad D=\frac{d}{d x} .
$$

From standard considerations [DKN], a matrix Lax pair formulation can be obtained without much difficulty if we take

$$
\begin{aligned}
\mathcal{L} & =D+q(x)+\Lambda \\
& =D+\left(\begin{array}{ccc}
0 & \ldots & u_{0}(x) \\
\vdots & & \vdots \\
0 & \ldots & u_{n-1}(x)
\end{array}\right)+\left(\begin{array}{cccc}
0 & 0 & \ldots & \lambda \\
1 & 0 & \ldots & 0 \\
& \ddots & \ddots & \\
0 & & 1 & 0
\end{array}\right)
\end{aligned}
$$

where $\lambda$ is a spectral parameter, in which case (1.11) becomes

$$
\dot{\mathcal{L}}=\left[\mathcal{A}_{k}, \mathcal{L}\right]
$$

However, an operator of the form $\mathcal{L}$ in (1.13) has no natural analogue in the case when $\operatorname{s\ell }\left(n, \mathbf{C}\left[\lambda, \lambda^{-1}\right]\right)$ is replaced by an arbitrary Kac-Moody algebra. This difficulty was overcome by Drinfeld and Sokolov in [DS], through the introduction of the following notion of gauge equivalence:

$$
\mathcal{L} \mapsto \widetilde{\mathcal{L}}=N \mathcal{L} N^{-1},
$$

where $N$ is a function which takes values in the group of upper triangular matrices with 1's on the diagonal. More precisely, they considered operators $D+q(x)+\Lambda$ where $q(x)$ is an upper triangular matrix, and associated equations in Lax pair form which preserve gauge equivalence. In this way, they were able to interpret (1.14) as an equation for the class of gauge equivalence. Furthermore, this point of view allows them to define $\mathrm{KdV}$ type equations for arbitrary Kac-Moody algebras. In particular, for a classical Kac-Moody algebra distinct from $s \ell\left(n, \mathbf{C}\left[\lambda, \lambda^{-1}\right]\right)$ and a vertex $c_{m}$ of its Dynkin diagram, they found that the associated generalized KdV equations can be realized in the form

$$
\begin{aligned}
& \dot{L}_{1}=A_{2} L_{1}-L_{1} A_{1}, \\
& \dot{L}_{2}=A_{1} L_{2}-L_{2} A_{2},
\end{aligned}
$$

where

$$
A_{1}=\sum_{i} b_{i}\left(\left(L_{2} L_{1}\right)^{\frac{2 i+1}{k}}\right)_{+}, A_{2}=\sum_{i} b_{i}\left(\left(L_{1} L_{2}\right)^{\frac{2 i+1}{k}}\right)_{+},
$$

$b_{i} \in \mathbf{C}, k=$ ord $L_{1}+$ ord $L_{2}$, and $L_{1}, L_{2}$ are appropriate differential or pseudodifferential operators. Thus equations analogous to (1.9) do arise in the theory of integrable PDEs. 
We now describe the main results of the paper. In section 2, we begin by introducing the Poisson structure of $(1.8)$. Let $(\cdot, \cdot)$ be the standard pairing on $\mathfrak{g}$, and let $R^{*}$ denote the dual of $R$ with respect to $(\cdot, \cdot)$. We have

Theorem A $[\mathrm{LP}]$. Equation (1.8) is the Hamilton equation generated by $H_{\varphi}=$ $\varphi \circ T$ in the Poisson structure

$$
\begin{aligned}
\left\{\psi_{1}, \psi_{2}\right\}=\frac{1}{2} \sum_{j=1}^{2} & \left(\left(A\left(D_{j}^{\prime} \psi_{1}\right), D_{j}^{\prime} \psi_{2}\right)-\left(A\left(D_{j} \psi_{1}\right), D_{j} \psi_{2}\right)\right. \\
& \left.+\left(S\left(D_{j} \psi_{1}\right), D_{j-1}^{\prime} \psi_{2}\right)-\left(S\left(D_{j-1}^{\prime} \psi_{1}\right), D_{j} \psi_{2}\right)\right)
\end{aligned}
$$

on $G^{2}$, where $A \equiv \frac{1}{2}\left(R-R^{*}\right)$ and $S \equiv \frac{1}{2}\left(R+R^{*}\right)$, and the subscript $j$ is taken mod 2 .

Note that in contrast to many well-known integrable systems, the Poisson structure here is nonlinear. As a matter of fact, it follows from a general result in [L2] that $\{$,$\} is an extension of a Sklyanin quadratic bracket \{,\}_{\text {Skly }}$ [STS1], [STS2] on $G$. More precisely, $\left(G,\{,\}_{\text {Skly }}\right)$ is a Poisson submanifold of $\left(G^{2},\{\},\right)$, although $\left(G^{2},\{\},\right)$ is not a Poisson Lie group in the sense of Drinfeld [D]. For reason explained in [L2], we shall call this Poisson structure the twisted structure. Our main result in section 2 is the description of generic symplectic leaves of the twisted structure. For $k=0, \ldots, n-1$, we introduce the polynomials

$$
\begin{aligned}
Q_{k}\left(g_{1}, g_{2}, \lambda\right) & \equiv \operatorname{det}\left(g_{1}-\lambda g_{2}^{-1}\right)_{k} \\
& =\sum_{r=0}^{n-k} F_{r k}\left(g_{1}, g_{2}\right) \lambda^{n-k-r}, \quad\left(g_{1}, g_{2}\right) \in G^{2},
\end{aligned}
$$

where $(M)_{k}$ denote the $(n-k) \times(n-k)$ matrix obtained from $M$ by deleting the first $k$ columns and last $k$ rows. Then we show that for $1 \leq k \leq n-1$, the quantities $\operatorname{sgn} F_{0 k}, \operatorname{sgn} F_{n-k, k}$ are constant on the symplectic leaves. To obtain symplectic leaves of maximal dimension, we restrict to those $g=\left(g_{1}, g_{2}\right) \in G^{2}$ for which

$$
\sigma\left(g_{1}, g_{2}^{T}\right)=\left\{\lambda \in \mathbb{C} \mid \operatorname{det}\left(g_{1}-\lambda g_{2}^{T}\right)=0\right\}
$$

is simple, i.e., has precisely $n$ distinct elements. Thus we consider the set

$$
\begin{array}{r}
\mathcal{U}=\left\{g=\left(g_{1}, g_{2}\right) \in G^{2} \mid F_{0 k}(g) \neq 0, F_{n-k, k}(g) \neq 0,\right. \\
\left.k=1, \ldots, n, \sigma\left(g_{1}, g_{2}^{T}\right) \text { is simple }\right\}
\end{array}
$$

which is an open, dense subset of $G^{2}$, foliated by symplectic leaves of the twisted structure. In order to describe these leaves in $\mathcal{U}$, we construct a rather specific collection of invariants of the group actions

$$
\left(g_{1}, g_{2}\right) \mapsto\left(h_{1} g_{1} h_{2}^{-1}, h_{2} g_{2} h_{1}^{-1}\right), \quad\left(h_{1} h_{2}\right) \in K^{2}\left(\text { resp. } L^{2}\right),
$$

from which we derive not only the Casmir functions, but also the Poisson commuting integrals of our lattice Lax systems. The $K^{2}$-invariants are given by the coefficients of the polynomial

$$
\begin{aligned}
J\left(g_{1}, g_{2} ; h, z\right) & \equiv \operatorname{det}\left(g_{2} g_{1}-h g_{2} g_{2}^{T}-z\right) \\
& =\sum_{r=0}^{n} \sum_{k=0}^{r} J_{r k}\left(g_{1}, g_{2}\right) h^{k} z^{n-r}
\end{aligned}
$$


while the $L^{2}$-invariants are

$$
I_{r k}\left(g_{1}, g_{2}\right)=F_{r k}\left(g_{1}, g_{2}\right) / F_{0 k}\left(g_{1}, g_{2}\right), \quad 1 \leq k \leq n-1,1 \leq r \leq n-k,
$$

for $\left(g_{1}, g_{2}\right) \in \mathcal{U}$. Let $\hat{I}_{n-k, k}(g)=(-1)^{n(n-k)} I_{n-k, k}(g) / \operatorname{det} g_{2}, 1 \leq k \leq n-1$, and $\hat{J}_{n k}(g)=J_{n k}(g) / \operatorname{det} g_{2}, 0 \leq k \leq n$.

Theorem B. Let $g^{0} \in \mathcal{U}$, and suppose $\mathcal{L}_{g^{0}}$ denotes the symplectic leaf of the twisted structure through $g^{0}$. Then

$$
\begin{array}{r}
\mathcal{L}_{g^{0}}=\left\{g \in \mathcal{U} \mid \operatorname{sgn} F_{0 k}(g)=\operatorname{sgn} F_{0 k}\left(g^{0}\right), \hat{I}_{n-k, k}(g)=\hat{I}_{n-k, k}\left(g^{0}\right),\right. \\
\left.1 \leq k \leq n-1, \hat{J}_{n k^{\prime}}(g)=\hat{J}_{n k^{\prime}}\left(g^{0}\right), \quad 0 \leq k^{\prime} \leq n\right\} .
\end{array}
$$

In particular, $\operatorname{dim} \mathcal{L}_{g^{0}}=2 n(n-1)$.

In section 3, we introduce variables which will turn out to be (essentially) the action-angle variables, and give their properties. The conserved quantities in involution are given by the $K^{2}$-invariants and $L^{2}$-invariants in $(1.20),(1.21)$. Note that the $I_{r k}$ 's are symmetric functions of the eigenvalues $\lambda_{r k}$ of the generalized eigenvalue problem $\left(g_{1}-\lambda g_{2}^{-1}\right)_{k} u=0, u \in \mathbf{C}^{n-k}$. Also, the spectral curve

$$
\mathcal{C}\left(g_{1}, g_{2}\right)=\left\{[u: h: z] \in \mathbf{C P}^{2} \mid \widetilde{J}\left(g_{1}, g_{2} ; u, h, z\right)=0\right\}
$$

is conserved by the flows. To introduce the angle variables corresponding to $\lambda_{r k}$ and $J_{r^{\prime} k^{\prime}}$, we make additional assumptions:

(GA1) All the $\lambda_{r k}$ 's are distinct, $1 \leq r \leq n-k, 0 \leq k \leq n-1$.

(GA2) Zero is a regular value of $J\left(g_{1}, g_{2} ; \cdot, \cdot\right)$ and $\sigma\left(g_{2}, g_{2}^{T}\right)$ is simple.

If $\left(g_{1}, g_{2}\right) \in \mathcal{U}$ satisfies (GA1), we adapt an idea in [DLNT] to our situation and consider the generalized eigenvalue problem $\left(g_{1}-\lambda_{r k} g_{2}^{-1}\right)_{k} w_{r k}=0$. From the assumption, the first component of $w_{r k}$ is non-zero and proportional to $m_{r k}\left(g_{1}, g_{2}\right)=\left(e_{2},\left(g_{1}-\lambda_{r k} g_{2}^{-1}\right)_{k-1} e_{n-k+1}\right)$. Hence we can define

$$
\theta_{r k}\left(g_{1}, g_{2}\right)=\lambda_{r k}^{-1} \log \left(m_{r k}\left(g_{1}, g_{2}\right) / m_{1 k}\left(g_{1}, g_{2}\right)\right), \quad 2 \leq r \leq n-k, 1 \leq k \leq n-1 .
$$

For $\left(g_{1}, g_{2}\right) \in \mathcal{U}$ satisfying (GA2), $\mathcal{C}\left(g_{1}, g_{2}\right)$ is a smooth projective curve. Since $g_{2} g_{1}-h g_{2} g_{2}^{T}$ undergoes an isospectral deformation when $\left(g_{1}, g_{2}\right)$ evolves under any of the $J_{r^{\prime}} k^{\prime}$-flows, a typical result $[\mathrm{AvM}],[\mathrm{RSTS}],[\mathrm{G}]$ says that for most of these systems, the flow can be linearized on the Jacobian variety of the curve via the Abel-Jacobi map. Thus we introduce the eigenvector map

$$
f: \mathcal{C}\left(g_{1}, g_{2}\right) \rightarrow \mathbf{C P}^{n-1}, \quad[u: h: z] \mapsto \operatorname{ker}\left(u g_{2} g_{1}-h g_{2} g_{2}^{T}-z\right),
$$

the divisor $D\left(g_{1}, g_{2}\right)=f^{*} H$ where $H$ is a suitable hyperplane in $\mathbf{C P}^{n-1}$ (we can take $H=\left\{\left[z_{1}: \cdots: z_{n}\right] \in \mathbf{C P}^{n-1} \mid z_{1}=0\right\}$ in our case), and the variables $\int_{D_{0}}^{D\left(g_{1}, g_{2}\right)} \omega_{r k}$ where $\left\{\omega_{r k}\right\}$ is a basis of $H^{0}\left(\mathcal{C}\left(g_{1}, g_{2}\right), \Omega\right)$. Indeed, we will take

$$
\omega_{r k}=\frac{h^{k} z^{n-r}}{J_{z}\left(g_{1}, g_{2} ; h, z\right)} d h, \quad 0 \leq k \leq r-3, r=3, \ldots, n,
$$

and define

$$
\phi_{r k}\left(g_{1}, g_{2}\right)=\int_{D_{0}\left(g_{1}, g_{2}\right)}^{D\left(g_{1}, g_{2}\right)} \omega_{r+k, k-1}, \quad 1 \leq k \leq r-1, r=2, \ldots, n-1,
$$

where $D_{0}\left(g_{1}, g_{2}\right)=\frac{n(n-1)}{2} P_{0}, P_{0}=[1: \tilde{h}: 0] \in \mathcal{C}\left(g_{1}, g_{2}\right)$. However, a simple count shows that in our case, $\operatorname{dim} H^{0}\left(\mathcal{C}\left(g_{1}, g_{2}\right), \Omega\right)$ is less than the number of nontrivial 
integrals from $\mathcal{C}\left(g_{1}, g_{2}\right)$; hence the Abel-Jacobi map is inadequate here. As a matter of fact, a direct calculation immediately confirms the existence of flows (namely, the $J_{r 0}$-flows and the $J_{r r}$-flows) that do not give rise to nontrivial motions on the Jacobian variety. The variables which move linearly under these flows, nevertheless, are algebra-geometric in nature. For the $J_{r 0}$-flows, we have to introduce the variables

$$
\phi_{r o}\left(g_{1}, g_{2}\right)=\int_{D_{0}\left(g_{1}, g_{2}\right)}^{D\left(g_{1}, g_{2}\right)} \omega_{r+1,-1}, \quad r=1, \ldots, n-1,
$$

where the differentials

$$
\omega_{r+1,-1}=\frac{z^{n-r-1}}{h J_{z}\left(g_{1}, g_{2} ; h, z\right)} d h
$$

are meromorphic with simple poles where $h=0$, and we refer the reader to Remark 4.19 for an interpretation of such quantities. Concerning the $J_{r r}$-flows, it turns out that the eigenvector map

$$
f^{T}: \mathcal{C}\left(g_{1}, g_{2}\right) \rightarrow \mathbf{C P}^{n-1}, \quad[u: h: z] \mapsto \operatorname{ker}\left(u g_{2} g_{1}-h g_{2} g_{2}^{T}-z\right)^{T}
$$

and the divisor $D^{T}\left(g_{1}, g_{2}\right)=\left(f^{T}\right)^{*} H$ also come into play. More precisely, the associated variables are given by

$$
\phi_{r r}\left(g_{1}, g_{2}\right)=\frac{1}{2} \int_{D^{T}\left(g_{1}, g_{2}\right)}^{D\left(g_{1}, g_{2}\right)} \omega_{r+1, r-1}, \quad r=1, \ldots, n-1,
$$

where the differentials

$$
\omega_{r+1, r-1}=\frac{h^{r-1} z^{n-r-1}}{J_{z}\left(g_{1}, g_{2} ; h, z\right)} d h
$$

are meromorphic with simple poles at the points "at $\infty$ ". The quantities introduced in $(1.24)-(1.26)$ are of course defined modulo a lattice $\Lambda$ in the space $\mathbf{C}^{g_{c}+2(n-1)}$ $\left(g_{c}=\right.$ genus of $\left.\mathcal{C}\left(g_{1}, g_{2}\right)\right)$, and we refer the reader to (3.16) for a detailed description. In section 4 , we compute the Poisson brackets of the variables introduced in section 3 . In the following theorem, the range of indices for $\theta_{r k}, \phi_{r k}, \phi_{r 0}$ and $\phi_{r r}$ are as indicated in (1.23) - (1.26); on the other hand, we only consider $\lambda_{r k}$ for $1 \leq k \leq$ $n-1,2 \leq r \leq n-k$, and we only consider $J_{r^{\prime} k^{\prime}}$ for $0 \leq k^{\prime} \leq r^{\prime}, 1 \leq r^{\prime} \leq n-1$.

Theorem C. At a point $\left(g_{1}, g_{2}\right) \in \mathcal{U}$ satisfying (GA1) and (GA2), we have the following Poisson bracket relations:

$$
\begin{gathered}
\left\{\lambda_{r k}, \lambda_{r^{\prime} k^{\prime}}\right\}=0, \quad\left\{J_{r k}, J_{r^{\prime} k^{\prime}}\right\}=0, \quad\left\{\lambda_{r k}, J_{r^{\prime} k^{\prime}}\right\}=0 ; \\
\left\{\theta_{r k}, \lambda_{r^{\prime} k^{\prime}}\right\}=\delta_{k k^{\prime}} \delta_{r r^{\prime}}, \quad\left\{\phi_{r k}, \lambda_{r^{\prime} k^{\prime}}\right\}=\left\{\phi_{r 0}, \lambda_{r^{\prime} k^{\prime}}\right\}=\left\{\phi_{r r}, \lambda_{r^{\prime} k^{\prime}}\right\}=0, \\
\left\{\phi_{r k}, J_{r^{\prime} k^{\prime}}\right\}=-\delta_{k k^{\prime}} \delta_{r r^{\prime}}, \quad\left\{\phi_{r 0}, J_{r^{\prime} k^{\prime}}\right\}=-\delta_{0 k^{\prime}} \delta_{r r^{\prime}}, \\
\left\{\phi_{r r}, J_{r^{\prime} k^{\prime}}\right\}=-\delta_{k^{\prime} r^{\prime}} \delta_{r r^{\prime}}, \quad\left\{\theta_{r k}, J_{r^{\prime} k^{\prime}}\right\}=0 ;
\end{gathered}
$$

Corollary D. The lattice Lax systems (1.8) are completely integrable on generic symplectic leaves of the twisted structure with integrals $\lambda_{r k}$ and $J_{r^{\prime} k^{\prime}}$. If $\left(g_{1}^{0}, g_{2}^{0}\right) \in \mathcal{U}$ satisfy (GA1) and (GA2), the level set of integrals $I\left(g_{1}^{0}, g_{2}^{0}\right)$ is a smooth manifold of dimension $n(n-1)$. 
Let $I^{c}\left(g_{1}^{0}, g_{2}^{0}\right)$ denote the connected component of $I^{c}\left(g_{1}^{0}, g_{2}^{0}\right)$ containing $\left(g_{1}^{0}, g_{2}^{0}\right)$. Then by replacing $\theta_{r k}$ by (essentialy) its real and imaginary parts, and $\phi_{r k}$ by $\phi_{r k}^{0}\left(g_{1}^{0}, g_{2}^{0}\right)=\int_{D_{0}\left(g_{1}^{0}, g_{2}^{0}\right)}^{D\left(g_{1}, g_{2}\right)} \omega_{r+k, k-1}, 0 \leq k \leq r-1$ (the $\phi_{r r}$ 's are already real-valued $\bmod \Lambda$ ), we obtain the linearization map

$$
\mathbf{L}^{0}: I^{c}\left(g_{1}^{0}, g_{2}^{0}\right) \rightarrow \mathbf{R}^{\frac{1}{2}(n-1)(n-2)-\sum_{i} c_{i}} \times \mathbb{T}^{\sum c_{i}} \times\left(\mathbf{R}^{\frac{1}{2}(n-1)(n-2)+2(n-1)} / \Lambda\right)
$$

into real variables (here $c_{k}=\#$ of complex conjugate pairs in $\left\{\lambda_{r k}\right\}_{1 \leq r \leq n-k}$ ). In Section 5, we prove

Theorem E. $\mathbf{L}^{0}$ is injective.

Finally, in Section 6, we solve the Hamilton equations generated by the conserved quantities via factorization problems. Explicitly, these equations are given respectively by

$$
\begin{aligned}
& \dot{g}_{1}=\left(\Pi_{\mathfrak{k}}\left(D_{1} I_{r k}\left(g_{1}, g_{2}\right)\right)\right) g_{1}-g_{1}\left(\Pi_{\mathfrak{k}}\left(D_{1}^{\prime} I_{r k}\left(g_{1}, g_{2}\right)\right)\right), \\
& \dot{g}_{2}=\left(\Pi_{\mathfrak{k}}\left(D_{1}^{\prime} I_{r k}\left(g_{1}, g_{2}\right)\right)\right) g_{2}-g_{2}\left(\Pi_{\mathfrak{k}}\left(D_{1} I_{r k}\left(g_{1}, g_{2}\right)\right)\right), \quad k \geq 1,
\end{aligned}
$$

and

$$
\begin{aligned}
\dot{g}_{1}= & \frac{1}{2} g_{1}\left(\Pi_{\mathfrak{r}}\left(D_{1}^{\prime} J_{r k}\left(g_{1}, g_{2}\right)+D_{2} J_{r k}\left(g_{1}, g_{2}\right)\right)\right) \\
& -\frac{1}{2}\left(\Pi_{\mathfrak{l}}\left(D_{1} J_{r k}\left(g_{1}, g_{2}\right)+D_{2}^{\prime} J_{r k}\left(g_{1}, g_{2}\right)\right)\right) g_{1}, \\
\dot{g}_{2}= & \frac{1}{2} g_{2}\left(\Pi_{\mathfrak{l}}\left(D_{2}^{\prime} J_{r k}\left(g_{1}, g_{2}\right)+D_{1} J_{r k}\left(g_{1}, g_{2}\right)\right)\right) \\
& -\frac{1}{2}\left(\Pi_{\mathfrak{l}}\left(D_{2} J_{r k}\left(g_{1}, g_{2}\right)+D_{1}^{\prime} J_{r k}\left(g_{1}, g_{2}\right)\right)\right) g_{2}, \quad k \geq 0 .
\end{aligned}
$$

Theorem F. The solutions of the $I_{r k}$-flows, $J_{r 0}$-flows, and $J_{r r}$-flows are obtained via factorization problems in the finite dimensional matrix group $G^{2}$.

As an example, suppose $Z_{i}(t) \in K, X_{i}(t) \in L, i=1,2$, are the solutions of the factorization problems $e^{-\frac{1}{2} D_{2} J_{r r}\left(g_{1}^{0}, g_{2}^{0}\right)}=Z_{1}(t) X_{1}(t), e^{-\frac{1}{2} D_{2}^{\prime} J_{r r}\left(g_{1}^{0}, g_{2}^{0}\right)}=Z_{2}(t) X_{2}(t)$. Then the $J_{r r}$-flow with initial conditions $g_{1}(0)=g_{1}^{0}, g_{2}(0)=g_{2}^{0}$ is given by $g_{1}(t)=$ $X_{2}(t) g_{1}^{0} X_{1}(t)^{-1}, g_{2}(t)=X_{1}(t) g_{2}^{0} X_{2}(t)^{-1}=Z_{1}^{T}(t) g_{2}^{0} Z_{2}(t)$. On the other hand, for $1 \leq k \leq r-1$, the $J_{r k}$-flows give rise to nondegenerate isospectral deformations of the matrix pencil $M_{h}\left(g_{1}, g_{2}\right)=g_{2}, g_{1}-h g_{2} g_{2}^{T}$. What is remarkable here is the fact that the isospectral deformations are generated by ad-invariant Hamiltonians with respect to a Poisson structure, where the $r$-matrix approach is feasible. Therefore, the Lax equations for $M_{h}\left(g_{1}, g_{2}\right)$ are solved via factorization problems in a loop group. To be more precise, consider the Lie algebra $g \ell_{n}\left(\mathbf{C}\left[h, h^{-1}\right]\right)$ with the pairing

$$
(X, Y)=\frac{1}{2 \pi \sqrt{-1}} \oint_{|h|=1} \operatorname{tr}(X(h) Y(h)) \frac{d h}{h} .
$$

Let

$$
\tilde{\mathfrak{l}}=\left\{X(h)=\sum_{j \geq 0} X_{j} h^{j} \in g \ell_{n}(\mathbf{C}[h]) \mid X_{0} \in \mathfrak{l}\right\}
$$

and

$$
\tilde{\mathfrak{k}}=\left\{X(h)=\sum_{j \leq 0} X_{j} h^{j} \in g \ell_{n}\left(\mathbf{C}\left[h^{-1}\right]\right) \mid X_{0} \in \mathfrak{k}\right\}
$$


Then $\tilde{\mathfrak{l}}$ and $\tilde{\mathfrak{k}}$ are subalgebras of $g \ell_{n}\left(\mathbf{C}\left[h, h^{-1}\right]\right)$ and we have $g \ell_{n}\left(\mathbf{C}\left[h, h^{-1}\right]\right)=\tilde{\mathfrak{k}} \oplus \tilde{\mathfrak{l}}$ (with associated projections $\Pi_{\tilde{\mathfrak{k}}}$ and $\Pi_{\tilde{\mathfrak{l}}}$ ). Therefore, $\widetilde{R}=\Pi_{\tilde{\mathfrak{k}}}-\Pi_{\tilde{\mathfrak{l}}}$ is an $r$-matrix. Let $\tilde{A}=\frac{1}{2}\left(\widetilde{R}-\widetilde{R}^{*}\right), S=\frac{1}{2}\left(\widetilde{R}+\widetilde{R}^{*}\right)$.

Theorem G. (a) For $1 \leq k \leq r-1$, the Hamilton equation generated by $J_{r k}$ with initial condition $\left(g_{1}(0), g_{2}(0)\right)=\left(g_{1}^{0}, g_{2}^{0}\right)$ gives rise to the isospectral deformation $\dot{X}=\left[X, \Pi_{\mathfrak{f}}\left(h^{-k} X(h) \nabla^{T} E_{r}(X(h))\right)\right]$ on $g \ell_{n}\left(\mathbf{C}\left[h, h^{-1}\right]\right)$ with initial condition $X(h, 0)=M_{h}\left(g_{1}^{0}, g_{2}^{0}\right)$ ( $E_{r}$ is the $r$-th elementary symmetric function).

(b) The isospectral deformation in (a) is the Hamilton equation generated by the ad-invariant function

$$
H_{r k}(X)=\frac{1}{2 \pi \sqrt{-1}} \oint_{|h|=1} E_{r}(X(h)) \frac{d h}{h^{k+1}}
$$

in the Poisson structure

$$
\begin{aligned}
\{F, H\}(X)= & \frac{1}{2}(\tilde{A}(d F(X) X), d H(X) X)-\frac{1}{2}(\tilde{A}(X d F(X)), X d H(X)) \\
& +\frac{1}{2}(\tilde{S}(X d F(X)), d H(X) X)-\frac{1}{2}(\tilde{S}(d F(X) X), X d H(X)) .
\end{aligned}
$$

(c) The solution of the initial value problem in (a) is given by

$$
X(h, t)=M_{h}\left(g_{1}(t), g_{2}(t)\right)=g_{ \pm}^{-1}(h, t) M_{h}\left(g_{1}^{0}, g_{2}^{0}\right) g_{ \pm}(h, t), \quad 0 \leq t<T,
$$

where $g_{ \pm}$are solutions of the factorization problem

$$
\exp \left\{t M_{h}\left(g_{1}^{0}, g_{2}^{0}\right) d H_{r k}\left(M_{h}\left(g_{1}^{0}, g_{2}^{0}\right)\right)\right\}=g_{+}(h, t) g_{-}(h, t)^{-1}, \quad h \in \mathbf{C P}^{1} \backslash\{0, \infty\},
$$

satisfying $g_{-}(\infty, t) \in K, g_{-}(h, t)^{-1} \dot{g}_{-}(h, t) \in \tilde{\mathfrak{k}}, h \in \mathbf{C P}^{1} \backslash\{0\}, g_{+}(0, t) \in L$, $g_{+}(h, t)^{-1} \dot{g}_{+}(h, t) \in \tilde{\mathfrak{l}}, h \in \mathbf{C P}^{1} \backslash\{\infty\}$. For $\left(g_{1}^{0}, g_{2}^{0}\right)$ satisfying (GA1) and (GA2), $g_{ \pm}(h, t)$ can be constructed by means of theta functions associated with the curve $\mathcal{C}\left(g_{1}^{0}, g_{2}^{0}\right)$. Lastly, we recover $g_{1}(t), g_{2}(t)$ using the information that $\lambda_{r k}\left(g_{1}(t), g_{2}(t)\right)$ $=\lambda_{r k}\left(g_{1}^{0}, g_{2}^{0}\right), 1 \leq r \leq n-k, 1 \leq k \leq n-1, \operatorname{det}\left(g_{2}(t)\right)_{n-k}=\operatorname{det}\left(g_{2}^{0}(t)\right)_{n-k}, k=$ $1, \ldots, n$.

In this paper, we have restricted our attention to the $r$-matrix associated with (1.4). If, in equation (1.8), we replace $R$ by the $r$-matrix associated with the LU decomposition or the Cholesky decomposition, the resulting Lax equations can also be shown to be completely integrable on generic symplectic leaves of the associated Poisson structures, but we provide no details here.

To conclude this introduction, let us remark that lattice Lax systems corresponding to $R$ and $\varphi$ on a periodic lattice with more than two sites are much more complicated. In this direction, preliminary investigation in the case where $N=3$ and $n=2$ already showed substantial differences from the case considered here, and we hope to report on this in future publications.

Some of the results stated above have been announced in [L1].

\section{Poisson structure and Generic symplectic leaves}

In this section, we describe the Poisson structure underlying equation (1.8) and describe its generic symplectic leaves.

Recall that $G$ is the identity component of the group of real, $n \times n$ invertible matrices. We shall equip its Lie algebra $\mathfrak{g}$ with the standard pairing

$$
(X, Y)=\operatorname{tr}(X Y), \quad X, Y \in \mathfrak{g} .
$$


For $\psi \in C^{\infty}(G)$, we define the left and right gradients $D^{\prime} \psi, D \psi: G \rightarrow \mathfrak{g}$ by

$$
\left(D^{\prime} \psi(g), X\right)=\left.\frac{d}{d t}\right|_{t=0} \psi\left(g e^{t X}\right),(D \psi(g), X)=\left.\frac{d}{d t}\right|_{t=0} \psi\left(e^{t X} g\right) .
$$

Since the pairing on $\mathfrak{g}$ can be naturally extended to $\mathfrak{g}_{2}=\mathfrak{g} \oplus \mathfrak{g}$ (Lie algebra direct sum), for a function $\Psi$ on $G^{2}=G \times G$, the left and right gradients can be defined as above, and we shall write $D^{\prime} \Psi=\left(D_{1}^{\prime}, \Psi, D_{2}^{\prime} \Psi\right), D \Psi=\left(D_{1} \Psi, D_{2} \Psi\right)$. Clearly,

$$
D_{i}^{\prime} \Psi(g)=\left(\nabla_{i} \Psi(g)\right)^{T} g_{i}, D_{i} \Psi(g)=g_{i}\left(\nabla_{i} \Psi(g)\right)^{T}, i=1,2,
$$

where $\nabla_{i} \Psi(g)$ is the gradient of $\Psi$ with respect to the $i$-th component $g_{i}$ of $g$. For the $r$-matrix $R$ defined in (1.6), we denote its dual with respect to the pairing $(\cdot, \cdot)$ by $R^{*}$. Set

$$
A=\frac{1}{2}\left(R-R^{*}\right)
$$

Then $R$ and $A$ are solutions of the modified Yang-Baxter equation. Hence we have

Theorem 2.5 [LP]. Equation (1.8) is the Hamilton equation generated by $H_{\varphi}=$ $\varphi \circ T$ in the Poisson structure

$$
\begin{aligned}
\left\{\psi_{1}, \psi_{2}\right\}=\frac{1}{2} \sum_{j=1}^{2}( & \left(A\left(D_{j}^{\prime} \psi_{1}\right), D_{j}^{\prime} \psi_{2}\right)-\left(A\left(D_{j} \psi_{1}\right), D_{j} \psi_{2}\right) \\
& \left.+\left(S\left(D_{j} \psi_{1}\right), D_{j-1}^{\prime} \psi_{2}\right)-\left(S\left(D_{j-1}^{\prime} \psi_{1}\right), D_{j} \psi_{2}\right)\right)
\end{aligned}
$$

on $G^{2}$, where $S \equiv \frac{1}{2}\left(R+R^{*}\right)$ and the subscript $j$ is taken $\bmod 2$.

Remark 2.7. Let $\pi_{2}$ be the permutation $\left(X_{1}, X_{2}\right) \mapsto\left(X_{2}, X_{1}\right)$ on $\mathfrak{g} \oplus \mathfrak{g}$, and denote by $\langle\cdot, \cdot\rangle$ the natural pairing on $\mathfrak{g} \oplus \mathfrak{g}$ induced by $(\cdot, \cdot)$ on $\mathfrak{g}$. Then the Poisson structure in (2.6) can also be expressed in the form

$$
\begin{aligned}
\left\{\psi_{1}, \psi_{2}\right\}= & \frac{1}{2}\left(\left\langle A\left(D^{\prime} \psi_{1}\right), D^{\prime} \psi_{2}\right\rangle-\left\langle A\left(D \psi_{1}\right), D \psi_{2}\right\rangle\right. \\
& \left.+\left\langle\pi_{2} \circ S\left(D \psi_{1}\right), D^{\prime} \psi_{2}\right\rangle-\left\langle\pi_{2} \circ S\left(D \psi_{2}\right), D^{\prime} \psi_{1}\right\rangle\right),
\end{aligned}
$$

where the operators $A$ and $S$ have been extended to $\mathfrak{g} \oplus \mathfrak{g}$. We shall call this the twisted structure, for reasons explained in [L2].

We now proceed to describe the generic symplectic leaves of this twisted structure. For $h, z \in \mathbf{C},\left(g_{1}, g_{2}\right) \in G^{2}$, let $M_{h, z}\left(g_{1}, g_{2}\right)=M_{h}\left(g_{1}, g_{2}\right)-z I$, where $M_{h}\left(g_{1}, g_{2}\right)=g_{2} g_{1}-h g_{2} g_{2}^{T}$, and define the polynomial

$$
\begin{aligned}
J\left(g_{1}, g_{2} ; h, z\right) & \equiv \operatorname{det} M_{h, z}\left(g_{1}, g_{2}\right) \\
& =\sum_{r=0}^{n} \sum_{k=0}^{r} J_{r k}\left(g_{1}, g_{2}\right) h^{k} z^{n-r} .
\end{aligned}
$$

Clearly, $J(\cdot, \cdot ; h, z)$ is invariant under the $K^{2}$ action in (1.16). The homogenization of $J\left(g_{1}, g_{2} ; h, z\right)$ will be denoted by $\widetilde{J}\left(g_{1}, g_{2} ; u, h, z\right)$; thus,

$$
\begin{aligned}
\widetilde{J}\left(g_{1}, g_{2} ; u, h, z\right) & \equiv \operatorname{det}\left(u g_{2} g_{1}-h g_{2} g_{2}^{T}-z I\right) \\
& =\sum_{r=0}^{n} \sum_{k=0}^{r} J_{r k}\left(g_{1}, g_{2}\right) u^{r-k} h^{k} z^{n-r} .
\end{aligned}
$$


For an $n \times n$ matrix $M$, let $(M)_{k}$ denote the $(n-k) \times(n-k)$ matrix obtained from $M$ by deleting the first $k$ columns and last $k$ rows. For $k=0, \ldots, n-1$, we introduce the polynomials

$$
\begin{aligned}
Q_{k}\left(g_{1}, g_{2}, \lambda\right) & \equiv \operatorname{det}\left(g_{1}-\lambda g_{2}^{-1}\right)_{k} \\
& =\sum_{r=0}^{n-k} F_{r k}\left(g_{1}, g_{2}\right) \lambda^{n-k-r},\left(g_{1}, g_{2}\right) \in G^{2} .
\end{aligned}
$$

From the definition of the $Q_{k}$ 's, it is clear that

$$
F_{n-k, k}\left(g_{1}, g_{2}\right)=\operatorname{det}\left(g_{1}\right)_{k} \text {. }
$$

Also,

$$
\begin{aligned}
F_{0 k}\left(g_{1}, g_{2}\right) & =(-1)^{n-k} \operatorname{det}\left(g_{2}^{-1}\right)_{k} \\
& =(-1)^{n(n-k)} \operatorname{det}\left(g_{2}\right)_{n-k} / \operatorname{det} g_{2} .
\end{aligned}
$$

Proposition 2.14. For each $1 \leq k \leq n-1$, the quantities $\operatorname{sgn} F_{0 k}, \operatorname{sgn} F_{n-k, k}$ are constant on the symplectic leaves of the twisted structure.

Proof. We shall prove the assertion for $F_{0 k}$. From (2.13), it is enough to show that the sign of $F_{k}\left(g_{1}, g_{2}\right)=\operatorname{det}\left(g_{2}\right)_{n-k}$ is constant on each symplectic leaf of $\{$,$\} .$ From the definition of $F_{k}$, we have $D_{1} F_{k}=D_{1}^{\prime} F_{k}=0, D_{2} F_{k}\left(g_{1}, g_{2}\right)=\left(\begin{array}{ccc}F_{k} I_{k} & 0 \\ * & 0\end{array}\right)$ and $D_{2} F_{k}\left(g_{1}, g_{2}\right)=\left(\begin{array}{cc}0 & 0 \\ * F_{k} I_{k}\end{array}\right)$ (here $I_{k}$ is the $k \times k$ identity matrix). So in particular, $D_{2} F_{k}\left(g_{1}, g_{2}\right)$ and $D_{2}^{\prime} F_{k}\left(g_{1}, g_{2}\right)$ are lower triangular matrices. Consequently, for any $\psi \in C^{\infty}\left(G_{2}\right)$, we find

$$
\left\{F_{k}, \psi\right\}=\frac{1}{2} F_{k} \operatorname{tr}\left\{\left(\begin{array}{cc}
0 & 0 \\
0 & I_{k}
\end{array}\right)\left(D_{1} \psi+D_{2}^{\prime} \psi\right)-\left(\begin{array}{cc}
I_{k} & 0 \\
0 & 0
\end{array}\right)\left(D_{2} \psi+D_{1}^{\prime} \psi\right)\right\} .
$$

This shows the sign of $F_{k}$ is constant along the trajectories of Hamiltonian vector fields. Since any two points on a symplectic leaf are connected by a piecewise smooth curve, each segment of which is the trajectory of a Hamiltonian vector field $[\mathrm{W}]$, the assertion follows.

Proposition 2.15. det $g_{2}$ is a Casimir function for the twisted structure.

Proof. First note that with the notation introduced in Remark 2.7, we can rewrite (2.8) in the following way:

$$
\begin{aligned}
\left\{\psi_{1}, \psi_{2}\right\}= & \frac{1}{2}\left\langle D^{\prime} \psi_{1}-\pi_{2}\left(D \psi_{1}\right), \Pi_{\mathfrak{l}}\left(D^{\prime} \psi_{2}+\pi_{2}\left(D \psi_{2}\right)\right)\right\rangle \\
& -\frac{1}{2}\left\langle D \psi_{2}-\pi_{2}\left(D^{\prime} \psi_{2}\right), \Pi_{\mathfrak{k}}\left(D \psi_{1}+\pi_{2}\left(D^{\prime} \psi_{1}\right)\right)\right\rangle,
\end{aligned}
$$

where the operators $\Pi_{\mathfrak{k}}$ and $\Pi_{\mathfrak{l}}$ have been extended to $\mathfrak{g} \oplus \mathfrak{g}$. Hence, for $\psi \in C^{\infty}\left(G^{2}\right)$, we have

$$
\left\{\psi, \operatorname{det} g_{2}\right\}=\frac{1}{2}\left\langle D^{\prime} \psi-\pi_{2}(D \psi), \operatorname{det} g_{2}(I, I)\right\rangle=0
$$

Let

$$
\sigma\left(g_{1}, g_{2}^{T}\right)=\left\{\lambda \in \mathbf{C} \mid \operatorname{det}\left(g_{1}-\lambda g_{2}^{T}\right)=0\right\} .
$$

Definition 2.18. If the set $\sigma\left(g_{1}, g_{2}^{T}\right)$ has precisely $n$ distinct elements, we say $\sigma\left(g_{1}, g_{2}^{T}\right)$ is simple. 
We now introduce the set

$$
\begin{array}{r}
\mathcal{U}=\left\{g=\left(g_{1}, g_{2}\right) \in G^{2} \mid F_{0 k}(g) \neq 0, F_{n-k, k}(g) \neq 0,\right. \\
\left.k=1, \ldots, n, \sigma\left(g_{1}, g_{2}^{T}\right) \text { is simple }\right\} .
\end{array}
$$

It is not hard to show that $\mathcal{U}$ is non-empty, and therefore is an open, dense subset of $G^{2}$.

Proposition 2.20. The functions $\widehat{J}_{n k}\left(g_{1}, g_{2}\right)=J_{n k}\left(g_{1}, g_{2}\right) / \operatorname{det} g_{2}, 0 \leq k \leq n$, are Casimir functions of the Poisson manifold $\left(G^{2},\{\},\right)$, and are functionally independent on $\mathcal{U}$.

Proof. Note that $\widehat{J}_{n n}\left(g_{1}, g_{2}\right)=(-1)^{n} \operatorname{det} g_{2}$ is independent of $g_{1}$, so it is clear that $\widehat{J}_{n k}, 0 \leq k \leq n$, are functionally independent on $\mathcal{U}$. To show that these functions are Casimir functions, let $\widehat{J}\left(g_{1}, g_{2} ; h\right)=J\left(g_{1}, g_{2} ; h, 0\right)=\operatorname{det}\left(g_{2} g_{1}-h g_{2} g_{2}^{T}\right), h \notin$ $\sigma\left(g_{1}, g_{2}^{T}\right)$. In view of Proposition 2.15, it is enough to show $\{\psi, \widehat{J}(\cdot, \cdot, h)\}=0$ for all $\psi \in C^{\infty}\left(G^{2}\right)$. To do this, we have to invoke the fact that $\widehat{J}$ is invariant under the $K^{2}$ action in (1.16). The upshot of this invariance property is that the matrices $D_{1}^{\prime} \widehat{J}\left(g_{1}, g_{2} ; h\right)-D_{2} \widehat{J}\left(g_{1}, g_{2} ; h\right)$ and $D_{2}^{\prime} \widehat{J}\left(g_{1}, g_{2} ; h\right)-D_{1} \widehat{J}\left(g_{1}, g_{2} ; h\right)$ are both symmetric. Thus, it follows from (2.16) that

$$
\begin{aligned}
2\{\psi, & \widehat{J}(\cdot, \cdot ; h)\}\left(g_{1}, g_{2}\right) \\
& =\left\langle D^{\prime} \psi-\pi_{2}(D \psi), \Pi_{\mathfrak{l}}\left(D^{\prime} \widehat{J}\left(g_{1}, g_{2} ; h\right)+\pi_{2}\left(D \widehat{J}\left(g_{1}, g_{2} ; h\right)\right)\right)\right\rangle .
\end{aligned}
$$

Now, by direct computation, we find

$$
\begin{aligned}
& D_{1}^{\prime} \widehat{J}\left(g_{1}, g_{2} ; h\right)+D_{2} \widehat{J}\left(g_{1}, g_{2} ; h\right) \\
& \quad=\widehat{J}\left(g_{1}, g_{2} ; h\right)\left(2 I+h M_{h}\left(g_{1}, g_{2}\right)^{-1} g_{2} g_{2}^{T}-h g_{2} g_{2}^{T}\left(M_{h}^{T}\left(g_{1}, g_{2}\right)\right)^{-1}\right),
\end{aligned}
$$

whereas

$$
\begin{aligned}
& D_{1} \widehat{J}\left(g_{1}, g_{2} ; h\right)+D_{2}^{\prime} \widehat{J}\left(g_{1}, g_{2} ; h\right) \\
& \quad=\widehat{J}\left(g_{1}, g_{2} ; h\right)\left(2 I+h g_{2}^{T} M_{h}\left(g_{1}, g_{2}\right)^{-1} g_{2}-h g_{2}^{T}\left(M_{h}^{T}\left(g_{1}, g_{2}\right)\right)^{-1} g_{2}\right) .
\end{aligned}
$$

Substituting these two expressions into $(*)$ and using the definition of $\Pi_{\mathfrak{l}}$ yields

$$
\begin{aligned}
2\{\psi, & \widehat{J}(\cdot, \cdot ; h)\}\left(g_{1}, g_{2}\right) \\
& =2 \widehat{J}\left(g_{1}, g_{2} ; h\right)\left\{\left(D_{1}^{\prime} \psi-D_{2} \psi, I\right)+\left(D_{2}^{\prime} \psi-D_{1} \psi, I\right)\right\} \\
& =0,
\end{aligned}
$$

as desired.

From Propositions 2.14 and 2.20, we now conclude that $\mathcal{U}$ is foliated by the symplectic leaves of the twisted structure. Moreover, as we shall see, the associated Poisson tensor has constant rank on $\mathcal{U}$. Since $\mathcal{U}$ is dense in $G^{2}$, we shall call the leaves on $\mathcal{U}$ generic symplectic leaves.

For $g \in \mathcal{U}$, define [L3]

$$
I_{r k}\left(g_{1}, g_{2}\right)=F_{r k}\left(g_{1}, g_{2}\right) / F_{0 k}\left(g_{1}, g_{2}\right), \quad 1 \leq k \leq n-1,1 \leq r \leq n-k .
$$

In [L3], it was shown that these are invariant under the $L^{2}$ action in (1.16).

Proposition 2.22. The functions $\widehat{I}_{n-k, k}(g)=(-1)^{n(n-k)} I_{n-k, k}(g) / \operatorname{det} g_{2}, 1 \leq$ $k \leq n-1$, are functionally independent Casimir functions for the twisted structure in $\mathcal{U}$. 
Proof. From Proposition 2.15, it is sufficient to show that $\left\{\psi, I_{n-k, k}\right\}=0$ for all $\psi \in C^{\infty}(\mathcal{U})$. To do so, rewrite $(2.8)$ as

$$
\begin{aligned}
\left\{\psi_{1}, \psi_{2}\right\} & =\frac{1}{2}\left\langle D \psi_{1}-\pi_{2}\left(D^{\prime} \psi_{1}\right), \Pi_{\mathfrak{k}}\left(D \psi_{2}+\pi_{2}\left(D^{\prime} \psi_{2}\right)\right)\right\rangle \\
& -\frac{1}{2}\left\langle D^{\prime} \psi_{2}-\pi_{2}\left(D \psi_{2}\right), \Pi_{\mathfrak{l}}\left(D^{\prime} \psi_{1}+\pi_{2}\left(D \psi_{1}\right)\right)\right\rangle .
\end{aligned}
$$

By the invariance properties of the $I_{r k}$ 's, the matrices $D_{1} I_{n-k, k}(g)-D_{2}^{\prime} I_{n-k, k}(g)$ and $D_{2} I_{n-k, k}(g)-D_{1}^{\prime} I_{n-k, k}(g)$ are strictly lower triangular. Thus, it follows from (2.23) that

$$
\begin{aligned}
\left\{\psi, I_{n-k, k}\right\} & =\frac{1}{2}\left\langle D \psi-\pi_{2}\left(D^{\prime} \psi\right), \Pi_{\mathfrak{k}}\left(D I_{n-k, k}+\pi_{2}\left(D^{\prime} I_{n-k, k}\right)\right)\right\rangle \\
& =\frac{(-1)^{n(n-k)}}{2} \operatorname{det} g_{2}\left\langle D \psi-\pi_{2}\left(D^{\prime} \psi\right), \Pi_{\mathfrak{k}}\left(D \widehat{I}_{n-k, k}+\pi_{2}\left(D^{\prime} \widehat{I}_{n-k, k}\right)\right)\right\rangle .
\end{aligned}
$$

But from the definition, it is not hard to see that

$$
\widehat{I}_{n-k, k}(g)=\operatorname{det}\left(g_{1}\right)_{k} / \operatorname{det}\left(g_{2}\right)_{n-k},
$$

so that $D_{1} \widehat{I}_{n-k, k}(g)+D_{2}^{\prime} \widehat{I}_{n-k, k}(g)$ and $D_{2} \widehat{I}_{n-k, k}(g)+D_{1}^{\prime} \widehat{I}_{n-k, k}(g)$ are both lower triangular. As a result, $\Pi_{\mathfrak{k}}\left(D \widehat{I}_{n-k, k}+\pi_{2}\left(D^{\prime} \widehat{I}_{n-k, k}\right)\right)=0$, which in turn implies $\left\{\psi, I_{n-k, k}\right\}=0$. To show that the $\widehat{I}_{n-k, k}$ 's are functionally independent, suppose

$$
\sum_{k=1}^{n-1} \alpha_{k}\left(\begin{array}{l}
\nabla_{1}^{T} \widehat{I}_{n-k, k}(g) \\
\nabla_{2}^{T} \widehat{I}_{n-k, k}(g)
\end{array}\right)=0, \quad \mathfrak{g} \in \mathcal{U} .
$$

If we multiply this expression on the right by $g=\left(g_{1}, g_{2}\right)$, we find that

$$
\sum_{k=1}^{n-1} \alpha_{k}\left(\begin{array}{cc|cc}
0 & 0 & \multicolumn{2}{|c}{*} \\
* & \widehat{I}_{n-k, k}(g) I_{n-k} & \multicolumn{2}{|c}{*} \\
\hline & * & 0 & 0 \\
& * & -\widehat{I}_{n-k, k}(g) I_{k}
\end{array}\right)=0 .
$$

In particular, this gives

$$
\sum_{k=1}^{n-1} \alpha_{k} \widehat{I}_{n-k, k}(g)\left(\begin{array}{llll}
0 & & & \\
& I_{n-k} & & \\
& & 0 & \\
& & & -I_{n-k}
\end{array}\right)=0 .
$$

As $\widehat{I}_{n-k, k}(g) \neq 0, k=1, \ldots, n-1$, the above linear system has only the trivial solution.

For $g^{\circ} \in \mathcal{U}$, let $\mathcal{L}_{g^{\circ}}$ denote the symplectic leaf of the twisted structure through $g^{\circ}$ and set

$$
\begin{array}{r}
\mathcal{C}_{g^{\circ}}=\left\{g \in \mathcal{U} \mid \operatorname{sgn} F_{0 k}(g)=\operatorname{sgn} F_{0 k}\left(g^{\circ}\right), \widehat{I}_{n-k, k}(g)=\widehat{I}_{n-k, k}\left(g^{\circ}\right)\right. \\
\left.1 \leq k \leq n-1, \widehat{J}_{n k^{\prime}}(g)=\widehat{J}_{n k^{\prime}}\left(g^{\circ}\right), 0 \leq k^{\prime} \leq n\right\} .
\end{array}
$$

Theorem 2.25. Let $g^{\circ} \in \mathcal{U}$. Then $\mathcal{L}_{g^{\circ}}=\mathcal{C}_{g^{\circ}}, \operatorname{dim} \mathcal{L}_{g^{\circ}}=2 n(n-1)$. 
Proof. If we express the twisted structure in the right invariant frame, the associated Hamiltonian operator $\eta: G^{2} \rightarrow$ End $(\mathfrak{g} \oplus \mathfrak{g})$ is given by

$$
\eta(g)=\frac{1}{2}\left(A d_{g} \circ A \circ A d_{g-1}-A+S \circ \pi_{2} \circ A d_{g-1}-A d_{g} \circ \pi_{2} \circ S\right) .
$$

Now, using the invariance properties of $\widehat{I}_{n-k, k}$ and $\widehat{J}_{n k^{\prime}}$ together with Propositions $2.20,2.22$, we can check that these Casimir functions are functionally independent on $\mathcal{U}$. Hence $\mathcal{C}_{g^{\circ}}$ is an $2 n(n-1)$ dimensional submanifold of $\mathcal{U}$ containing $\mathcal{L}_{g^{\circ}}$. So $2 n \leq \operatorname{dim} \operatorname{ker} \eta\left(g^{\circ}\right)$. On the other hand, according to Propositions 4.6-4.9, 4.11-4.13, there exist $n(n-1)$ nontrivial integrals in involution with respect to the twisted structure and functionally independent over an open, dense subset of $\mathcal{L}_{g^{\circ}}$. In particular, this implies $n(n-1) \leq \frac{1}{2}\left(2 n^{2}-\operatorname{dim} \operatorname{ker} \eta\left(g^{\circ}\right)\right)$, i.e. $2 n \geq$ dim ker $\eta\left(g^{\circ}\right)$. Combining the two inequalities, we conclude that dim ker $\eta\left(g^{\circ}\right)=$ $2 n$. Hence the rank of $\eta(g)$ is constant $(=2 n(n-1))$ on $\mathcal{U}$, and therefore $\mathcal{L}_{g^{\circ}}=$ $\mathcal{C}_{g^{\circ}}$.

\section{ACTION-ANGLE VARIABLES AND THEIR PROPERTIES}

We now introduce a set of variables which will turn out to be (essentially) the action-angle variables for our lattice Lax systems.

The integrals naturally fall into two categories (with non-empty intersection) according to their invariance properties. The first set has already made its appearance in [L3]. Let $\lambda_{r k}=\lambda_{r k}\left(g_{1}, g_{2}\right), 1 \leq r \leq n-k$, be the roots of the equation $Q_{k}\left(g_{1}, g_{2} ; \lambda\right)=0,0 \leq k \leq n-1,\left(g_{1}, g_{2}\right) \in \mathcal{U}$. They are clearly the eigenvalues of the generalized eigenvalue problem

$$
\left(g_{1}-\lambda_{r k} g_{2}^{-1}\right)_{k} u=0, \quad u \in \mathbf{C}^{n-k} .
$$

Due to ambiguity in labelling the $\lambda_{r k}$ 's globally, these are locally smooth functions. The second set of invariants comes from the coefficients $J_{r^{\prime} k^{\prime}}$ of the polynomial $J\left(g_{1}, g_{2} ; h, z\right)$ introduced in $(2.9)$, so that there is an associated affine curve

$$
\mathcal{C}_{a}\left(g_{1}, g_{2}\right)=\left\{(h, z) \in \mathbf{C}^{2} \mid J\left(g_{1}, g_{2} ; h, z\right)=0\right\} .
$$

To introduce the angle variables, we make additional assumptions:

(GA1) all the $\lambda_{r k}$ 's are distinct, $1 \leq r \leq n-k, 0 \leq k \leq n-1$,

$(\mathrm{GA} 2)_{1}$ zero is a regular value of $J\left(g_{1}, g_{2} ; \cdot, \cdot\right)$,

$(\mathrm{GA} 2)_{2} \quad \sigma\left(g_{2} g_{2}^{T}\right)$ is simple.

If $\left(g_{1}, g_{2}\right) \in \mathcal{U}$ satisfies (GA1), we can define

$$
m_{r k}\left(g_{1}, g_{2}\right)=\left(e_{2},\left(g_{1}-\lambda_{r k} g_{2}^{-1}\right)_{k-1}^{-1} e_{n-k+1}\right), 1 \leq r \leq n-k, 1 \leq k \leq n-1,
$$

where $e_{2}, e_{n-k+1}$ are in the canonical basis of $\mathbf{C}^{n-k+1}$. As explained in [L3], $m_{r k}\left(g_{1}, g_{2}\right)$ is the first component of a suitably normalized eigenvector of the generalized eigenvalue problem (3.1). Furthermore, the variables $m_{r k}\left(g_{1}, g_{2}\right)$ are nonzero. Hence we can define

$$
\theta_{r k}\left(g_{1}, g_{2}\right)=\lambda_{r k}^{-1} \log \left(m_{r k}\left(g_{1}, g_{2}\right) / m_{1 k}\left(g_{1}, g_{2}\right)\right), 2 \leq r \leq n-k, 1 \leq k \leq n-1 .
$$

On the other hand, if $\left(g_{1}, g_{2}\right) \in \mathcal{U}$ satisfies (GA2), the affine curve $\mathcal{C}_{a}\left(g_{1}, g_{2}\right)$ is smooth by $(\mathrm{GA} 2)_{1}$. Let $\mathcal{C}\left(g_{1}, g_{2}\right)$ denote the corresponding projective curve, i.e.

$$
\mathcal{C}\left(g_{1}, g_{2}\right)=\left\{[u: h: z] \in \mathbf{C P}^{2} \mid \widetilde{J}\left(g_{1}, g_{2} ; u, h, z\right)=0\right\} .
$$


Then from $(\mathrm{GA} 2)_{2}, \mathcal{C}\left(g_{1}, g_{2}\right)$ is also smooth. As $\widetilde{J}$ has degree $n$, the genus of $\mathcal{C}\left(g_{1}, g_{2}\right)$ is given by $g_{c}=(n-1)(n-2) / 2$. Its $n$ points on the line $u=0$ are $P_{-}^{i}=\left[0:-1: \mu_{i}\right], 1 \leq i \leq n$, and will be considered as points "at $\infty$ ". Note that in contrast to the spectral curve associated with the Toda flows [DLT], $\mathcal{C}\left(g_{1}, g_{2}\right)$ is quite general. Let $u g_{2} g_{1}-h g_{2} g_{2}^{T}-z$ be denoted by $M\left(g_{1}, g_{2} ; p\right), p=[u: h: z] \in$ $\mathcal{C}\left(g_{1}, g_{2}\right)$. We now define a pair of holomorphic embeddings of $\mathcal{C}\left(g_{1}, g_{2}\right)$ in $\mathbf{C P}^{n-1}$. For $p \in \mathcal{C}\left(g_{1}, g_{2}\right)$, define

$$
f\left(g_{1}, g_{2} ; p\right)=\operatorname{ker} M\left(g_{1}, g_{2} ; p\right), f^{T}\left(g_{1}, g_{2} ; p\right)=\operatorname{ker}\left(M\left(g_{1}, g_{2} ; p\right)\right)^{T} .
$$

By (GA2), dim ker $M\left(g_{1}, g_{2} ; p\right)=\operatorname{dim} \operatorname{ker}\left(M\left(g_{1}, g_{2} ; p\right)\right)^{T}=1$, as the adjoint matrix adj $M\left(g_{1}, g_{2} ; p\right)$ of $M\left(g_{1}, g_{2} ; p\right)$ is non-zero and has rank 1 .

Proposition 3.7. If $\left(g_{1}, g_{2}\right) \in \mathcal{U}$ satisfies (GA2), then the kernel maps $f\left(g_{1}, g_{2} ; \cdot\right)$, $f^{T}\left(g_{1}, g_{2} ; \cdot\right): \mathcal{C}\left(g_{1}, g_{2}\right) \rightarrow \mathbf{C P}^{n-1}$ are holomorphic.

Proof. Let $e_{j}(p)$ denote the $j$-th column of adj $M\left(g_{1}, g_{2} ; p\right)$ and set $U_{j}=\{p \in$ $\left.\mathcal{C}\left(g_{1}, g_{2}\right) \mid e_{j}(p) \neq 0\right\}$. Then $\mathcal{C}\left(g_{1}, g_{2}\right)=\bigcup_{j=1}^{n} U_{j}$, and $f\left(g_{1}, g_{2} ; \cdot\right) \mid U_{j}: p \mapsto e_{j}(p)$ is polynomial and homogeneous. On $U_{j} \cap U_{k}$ it is clear that $e_{j}(p)=g_{j k}(p) e_{k}(p)$, where $g_{j k}: U_{j} \cap U_{k} \rightarrow \mathbf{C}^{*}$ is holomorphic. This shows $f\left(g_{1}, g_{2} ; \cdot\right): \mathcal{C}\left(g_{1}, g_{2}\right) \rightarrow \mathbf{C P}^{n-1}$ is holomorphic. The proof for $f^{T}\left(g_{1}, g_{2} ; \cdot\right)$ is similar.

From now on, whenever the pair $\left(g_{1}, g_{2}\right)$ is evident in the discussion, we will denote the kernel maps simply by $f$ and $f^{T}$. Consider the hyperplane

$$
H=\left\{\left[z_{1}: \ldots: z_{n}\right] \in \mathbf{C P}^{n-1} \mid z_{1}=0\right\} .
$$

Then $f\left(\mathcal{C}\left(g_{1}, g_{2}\right)\right) \not \subset H$, for otherwise, the vector $e_{1}$ in the canonical basis of $\mathbf{C}^{n}$ would be orthogonal to the eigenvectors $f\left(P_{-}^{i}\right), i=1, \ldots, n$, of the matrix $g_{2} g_{2}^{T}$. Similarly, $f^{T}\left(\mathcal{C}\left(g_{1}, g_{2}\right)\right) \not \subset H$. Therefore, the divisors

$$
D\left(g_{1}, g_{2}\right)=f^{*} H, \quad D^{T}\left(g_{1}, g_{2}\right)=\left(f^{T}\right)^{*} H
$$

are well-defined. Now, the divisor of zeros of $\left(e_{1}\right.$, adj $\left.M\left(g_{1}, g_{2} ; p\right) e_{1}\right)$ is given by $D\left(g_{1}, g_{2}\right)+D^{T}\left(g_{1}, g_{2}\right)$. By Bezout's theorem, it follows that $\operatorname{deg} D\left(g_{1}, g_{2}\right)+$ $\operatorname{deg} D^{T}\left(g_{1}, g_{2}\right)=n(n-1)$, and consequently, $\operatorname{deg} D\left(g_{1}, g_{2}\right)=\operatorname{deg} D^{T}\left(g_{1}, g_{2}\right)=$ $n(n-1) / 2$ as $M\left(g_{1}, g_{2} ; p\right)$ and $\left(M\left(g_{1}, g_{2} ; p\right)\right)^{T}$ are of equal standing. On the affine part of the curve $\mathcal{C}\left(g_{1}, g_{2}\right)$ set $u=1$; we obtain the coordinate functions $h$ and $z$ which extend to meromorphic functions on $\mathcal{C}\left(g_{1}, g_{2}\right)$. Furthermore,

$$
(h)=P_{+}-P_{-} \text {, }
$$

where $P_{ \pm}$are effective divisors of degree $n$ and

$$
P_{ \pm}=\sum_{i=1}^{n} P_{ \pm}^{i}
$$

Let

$$
\omega_{r k}=\omega_{r k}\left(g_{1}, g_{2}\right)=\frac{h^{k} z^{n-r}}{J_{z}\left(g_{1}, g_{2} ; h, z\right)} d h .
$$

Proposition 3.13. For $\left(g_{1}, g_{2}\right) \in \mathcal{U}$ satisfying (GA2),

(a) the space $H^{\circ}\left(\mathcal{C}\left(g_{1}, g_{2}\right), \Omega\right)$ of holomorphic 1-forms on $\mathcal{C}\left(g_{1}, g_{2}\right)$ is spanned by $\omega_{r k}, 0 \leq k \leq r-3, r=3, \ldots, n$, 
(b) the 1-forms

$$
\omega_{n-j,-1}=\frac{z^{j}}{h J_{z}\left(g_{1}, g_{2} ; h, z\right)} d h,
$$

$j=0, \ldots, n-2$, are meromorphic with poles only at $P_{+}^{i}, i=1, \ldots, n$,

(c) the 1-forms

$$
\omega_{k+1, k-1}=\frac{h^{k-1} z^{n-k-1}}{J_{z}\left(g_{1}, g_{2} ; h, z\right)} d h,
$$

$k=1, \ldots, n-1$, are meromorphic with poles only at $P_{-}^{i}, i=1, \ldots, n$.

Proof. (a) The proof is standard. By (GA2), all we need to check is regularity at the points $P_{-}^{i}, i=1, \ldots, n$.

(b) The 1-forms $\omega_{n-j,-1}$ have no poles on the affine part of $\mathcal{C}\left(g_{1}, g_{2}\right)$ except where $h=0$, i.e. at the points $P_{+}^{i}=\left[1: 0: \lambda_{i}\right], i=1, \ldots, n$. Near $P_{-}^{i}=[0:-1:$ $\left.\mu_{i}\right]$,

$$
\begin{aligned}
\frac{z^{j}}{h J_{z}\left(g_{1}, g_{2} ; h, z\right)} d h & \sim \text { const } \cdot \frac{\mu_{i}^{j} h^{j}}{h^{n} \prod_{k \neq i}\left(\mu_{k}-\mu_{i}\right)} d h \\
& =\text { const } \cdot u^{n-j-1} d u, \quad h=1 / u
\end{aligned}
$$

and so $\omega_{n-j,-1}$ is regular at $P_{-}^{i}$, as $j \leq n-2$.

(c) It is clear that the 1 -forms $\omega_{k+1, k-1}, k=1, \ldots, n-1$, have no poles on the affine part of $\mathcal{C}\left(g_{1}, g_{2}\right)$. Near $P_{-}^{i}=\left[0:-1: \mu_{i}\right]$,

$$
\begin{aligned}
\frac{h^{k+1} z^{n-k-1}}{J_{z}\left(g_{1}, g_{2} ; h, z\right)} d h & \sim(-1)^{n-k} \frac{\mu_{i}^{n-k-1}}{\Pi_{j \neq i}\left(\mu_{i}-\mu_{j}\right)} \cdot \frac{d h}{h} \\
& =\frac{(-1)^{n-k-1} \mu_{i}^{n-k-1}}{\Pi_{j \neq i}\left(\mu_{i}-\mu_{j}\right)} \cdot \frac{d u}{u}, \quad h=1 / u,
\end{aligned}
$$

and so $\omega_{k+1, k-1}$ has a simple pole at $P_{-}^{i}$. The above computation also shows that

$$
\operatorname{res}_{P_{-}^{i}} \omega_{k+1, k-1}=\frac{(-1)^{n-k-1} \mu_{i}^{n-k-1}}{\prod_{j \neq i}\left(\mu_{i}-\mu_{j}\right)} .
$$

By Proposition 2.20, the elements of $\sigma\left(g_{1}, g_{2}^{T}\right)$ are constant on the symplectic leaves of the twisted structure. Choose an $\tilde{h} \in \sigma\left(g_{1}, g_{2}^{T}\right)$ and set

$$
P_{0}=[1: \tilde{h}: 0], D_{0}\left(g_{1}, g_{2}\right)=\frac{n(n-1)}{2} P_{0} .
$$

For $\left(g_{1}, g_{2}\right) \in \mathcal{U}$ satisfying both (GA1) and (GA2), we define

$$
\begin{aligned}
& \phi_{r k}\left(g_{1}, g_{2}\right) \equiv \int_{D_{0}\left(g_{1}, g_{2}\right)}^{D\left(g_{1}, g_{2}\right)} \omega_{r+1, k-1}, \quad 1 \leq k \leq r-1, r=2, \ldots, n-1, \\
& \phi_{r 0}\left(g_{1}, g_{2}\right) \equiv \int_{D_{0}\left(g_{1}, g_{2}\right)}^{D\left(g_{1}, g_{2}\right)} \omega_{r+1,-1}, \quad r=1, \ldots, n-1, \\
& \phi_{r r}\left(g_{1}, g_{2}\right) \equiv \frac{1}{2} \int_{D^{T}\left(g_{1}, g_{2}\right)}^{D\left(g_{1}, g_{2}\right)} \omega_{r+1, r-1}, \quad r=1, \ldots, n-1 .
\end{aligned}
$$


In (3.15b), the paths of integration going from points in $D_{0}$ to points in $D\left(g_{1}, g_{2}\right)$ have to avoid the points $P_{+}^{i}, i=1, \ldots, n$. These multi-valued variables $\phi_{r 0}$ are well defined because none of the points in $D_{0}\left(g_{1}, g_{2}\right)$ and $D\left(g_{1}, g_{2}\right)$ can belong to $\left\{P_{+}^{i} \mid i=1, \ldots, n\right\}$ by the invertibility of $g_{1}$ and (GA1). Now, it is possible that $P_{-}^{i} \in \operatorname{supp} D\left(g_{1}, g_{2}\right)$ or $\operatorname{supp} D^{T}\left(g_{1}, g_{2}\right)$ for some $i$. But note that since $P_{-}^{i} \in$ supp $D\left(g_{1}, g_{2}\right) \Leftrightarrow P_{-}^{i} \in \operatorname{supp} D^{T}\left(g_{1}, g_{2}\right)$, the variables $\phi_{r r}$ are defined provided the paths of integration going from points in supp $D^{T}\left(g_{1}, g_{2}\right) \cap \mathcal{C}_{a}\left(g_{1}, g_{2}\right)$ to points in supp $D\left(g_{1}, g_{2}\right) \cap \mathcal{C}_{a}\left(g_{1}, g_{2}\right)$ are chosen to stay entirely in $\mathcal{C}_{a}\left(g_{1}, g_{2}\right)$. The variables introduced in (3.15) above are defined modulo the lattice $\Lambda$ in the space $\mathbf{C}^{g_{c}+2(n-1)}$ generated by column vectors of the $\left(g_{c}+2(n-1)\right) \times\left(2 g_{c}+n\right)$ matrix

$$
L=\left(\begin{array}{ccc}
L_{1} & 0 & 0 \\
L_{2} & L_{3} & 0 \\
L_{4} & 0 & L_{5}
\end{array}\right)
$$

where

(i) $L_{1}$ is the $g_{c} \times 2 g_{c}$ period matrix corresponding to the basis $\left\{\omega_{r k} \mid 0 \leq k \leq\right.$ $r-3, k=3, \ldots, n\}$ of $H^{\circ}\left(\mathcal{C}\left(g_{1}, g_{2}\right), \Omega\right)$ and a canonical basis $\delta_{1}, \ldots, \delta_{2 g_{c}}$ of $H_{1}\left(\mathcal{C}\left(g_{1}, g_{2}\right), \mathbf{Z}\right)$

(ii) $L_{2}$ is the $(n-1) \times 2 g_{c}$ matrix whose $(i, j)$ entry is $\oint_{\delta j} \omega_{i+1,-1}$,

(iii) $L_{3}$ is the $(n-1) \times n$ matrix whose $(i, j)$ entry is $\oint_{\alpha j} \omega_{i+1,-1}$, where $\alpha_{j}$ is a small simple closed contour enclosing the pole $P_{+}^{j}=\left[1: 0: \lambda_{j}\right]$ of the $\omega_{i+1,-1}$ 's,

(iv) $L_{4}$ is the $(n-1) \times 2 g_{c}$ matrix whose $(i, j)$ entry is $\oint_{\delta j} \omega_{i+1, i-1}$,

(v) $L_{5}$ is the $(n-1) \times n$ matrix whose $(i, j)$ entry is $\oint_{\beta j} \omega_{i+1, i-1}$, where $\beta_{j}$ is a small simple closed contour enclosing the pole $P_{-}^{j}=\left[0:-1: \mu_{j}\right]$ of the $\omega_{i+1, i-1}$ 's.

By the residue theorem, we have explicitly that

$$
\begin{aligned}
& \oint_{\alpha_{j}} \omega_{i+1,-1}=2 \pi \sqrt{-1} \frac{\lambda_{j}^{n-i-1}}{J_{z}\left(g_{1}, g_{2} ; 0, \lambda_{j}\right)}, \\
& \oint_{\beta_{j}} \omega_{i+1, i-1}=2 \pi \sqrt{-1} \frac{\mu_{j}^{n-i-1}}{\tilde{J}_{z}\left(g_{1}, g_{2} ; 0,-1, \mu_{j}\right)} .
\end{aligned}
$$

Since the sum of residues of the meromorphic 1-forms $\omega_{i+1,-1}, \omega_{i+1, i-1}$ is zero, it follows that the vector $\mathbf{1}=(1, \ldots, 1)^{T} \in \mathbf{C}^{n}$ belongs to both ker $L_{3}$ and ker $L_{5}$. By a Vandermonde type argument, we can actually show that ker $L_{3}=$ ker $L_{5}=$ span 1. Consequently, $\Lambda$ is a lattice of rank $2 g_{c}+2(n-1)=2\left(g_{c}+n-1\right)$.

At this juncture, it is convenient to count the number of variables. The integrals defined on generic symplectic leaves $\mathcal{L}_{\left(g_{1}, g_{2}\right)}$, where $\left(g_{1}, g_{2}\right) \in \mathcal{U}$ satisfies (GA1) and (GA2), are given by

$$
\left\{\begin{array}{lll}
\lambda_{r k}, & k=1, \ldots, n-1 ; & r=2, \ldots, n-k \\
J_{r^{\prime} k^{\prime}}, & k^{\prime}=0, \ldots, r^{\prime} ; & r^{\prime}=1, \ldots, n-1 .
\end{array}\right.
$$

Therefore, the total number of integrals equals

$$
\sum_{k=1}^{n-1}(n-k-1)+\sum_{r=1}^{n-1}(r+1)=n(n-1) .
$$


The conjugate variables for these integrals are constructed from

$$
\begin{cases}\theta_{r k}, & 2 \leq r \leq n-k, \quad 1 \leq k \leq n-1, \\ \phi_{r^{\prime} k^{\prime}}, & 2 \leq r^{\prime} \leq n-1, \quad 1 \leq k^{\prime} \leq r^{\prime}-1 \\ \phi_{r 0}, & 1 \leq r \leq n-1, \\ \phi_{r r}, & 1 \leq r \leq n-1 .\end{cases}
$$

Again, this adds up to $n(n-1)$.

Next, we describe the invariance properties of the various quantities that will play a role in the computation of Poisson brackets in the next section. As the variables $\lambda_{r k}$ and $\theta_{r k}$ have already been discussed in detail in [L3], we shall restrict ourselves to quantities associated with the spectral curve here.

Proposition 3.18. Let $\left(g_{1}, g_{2}\right) \in \mathcal{U}$ satisfy (GA1) and (GA2). Then

(a) $J_{r k}\left(h_{1} g_{1} h_{2}^{-1}, h_{2} g_{2} h_{1}^{-1}\right)=J_{r k}\left(g_{1}, g_{2}\right)$ for $h_{1}, h_{2} \in K, 0 \leq k \leq r, 1 \leq r \leq n-1$,

(b) $\phi_{r k}\left(h_{1} g_{1} h_{2}^{-1}, h_{2} g_{2} h_{1}^{-1}\right)=\phi_{r k}\left(g_{1}, g_{2}\right)$ for $h_{1}, h_{2} \in K, 1 \leq k \leq r-1,2 \leq r \leq$ $n-1$,

(c) $\phi_{r 0}\left(h_{1} g_{1} h_{2}^{-1}, h_{2} g_{2} h_{1}^{-1}\right)=\phi_{r 0}\left(g_{1}, g_{2}\right)$ for $h_{1} \in K, h_{2} \in \widehat{K}=\left\{k \in K \mid k e_{1}=\right.$ $\left.e_{1}\right\}$ and $h_{1}, h_{2}$ sufficiently close to the identity, $1 \leq r \leq n-1$,

(d) $\phi_{r r}\left(h_{1} g_{1} h_{2}^{-1}, h_{2} g_{2} h_{1}^{-1}\right)=\phi_{r r}\left(g_{1}, g_{2}\right), h_{1} \in K, h_{2} \in \widehat{K}, 1 \leq r \leq n-1$.

Proof. (a) This is obvious.

(b) Let $f$ be the kernel map defined earlier and introduce the meromorphic function $m, m(p)=\left(e_{1}, h_{2} f(p)\right) /\left(e_{1}, f(p)\right)$, where $h_{2} \in K$. We have

$$
\begin{aligned}
\phi_{r k}\left(g_{1}, g_{2}\right)= & \int_{D_{0}\left(g_{1}, g_{2}\right)}^{D\left(g_{1}, g_{2}\right)} \omega_{r+1, k-1} \\
= & \int_{D_{0}\left(g_{1}, g_{2}\right)}^{D_{0}\left(h_{1} g_{1} h_{2}^{-1}, h_{2} g_{2} h_{1}^{-1}\right)} \omega_{r+1, k-1}+\int_{D_{0}\left(h_{1} g_{1} h_{2}^{-1}, h_{2} g_{2} h_{1}^{-1}\right)}^{D\left(h_{1} g_{1} h_{2}^{-1}, h_{2} g_{2} h_{1}^{-1}\right)} \omega_{r+1, k-1} \\
& +\int_{D\left(h_{1} g_{1} h_{2}^{-1}, h_{2} g_{2} h_{1}^{-1}\right)}^{D\left(g_{1}, g_{2}\right)} \omega_{r+1, k-1},
\end{aligned}
$$

and the first term on the right hand side in the above expression is zero as $D_{0}\left(g_{1}, g_{2}\right)$ $=D_{0}\left(h_{1} g_{1}, h_{2}^{-1}, h_{2} g_{2} h_{1}^{-1}\right)$. Since $(m)=D\left(h_{1} g_{1} h_{2}^{-1}, h_{2} g_{2} h_{1}^{-1}\right)-D\left(g_{1}, g_{2}\right)$, it follows from Abel's theorem that the last term in the above expression is also zero.

(c) Clearly, the stipulation that $h_{1}, h_{2}$ are close to the identity is to ensure that $\left(h_{1} g_{1} h_{2}^{-1}, h_{2} g_{2} h_{1}^{-1}\right) \in \mathcal{U}$ and satisfies (GA1), so that $\phi_{r 0}\left(h_{1} g_{1} h_{2}^{-1}, h_{2} g_{2} h_{1}^{-1}\right)$ is defined. From the definition of $\widehat{K}$, we have $\left(e_{1}, h_{2} f(p)\right)=\left(e_{1}, f(p)\right)$ for $h_{2} \in \widehat{K}$, which implies $D\left(h_{1} g_{1} h_{2}^{-1}, h_{2} g_{2} h_{1}^{-1}\right)=D\left(g_{1}, g_{2}\right)$. The invariance of $\phi_{r 0}$ is now obvious.

(d) This follows from the invariance of the divisors $D\left(g_{1}, g_{2}\right)$ and $D^{T}\left(g_{1}, g_{2}\right)$ under the action $\left(g_{1}, g_{2}\right) \mapsto\left(h_{1} g_{1} h_{2}^{-1}, h_{2} g_{2} h_{1}^{-1}\right), h_{1} \in K, h_{2} \in \widehat{K}$.

Now, as in Proposition 2.14 of [DLT], we can show that the functions $\theta_{r k}, \phi_{r k}$ are smooth on the open set of matrices which lie in $\mathcal{U}$ and satisfy (GA1), (GA2). Hence we have

Corollary 3.19. Let $\left(g_{1}, g_{2}\right) \in \mathcal{U}$ satisfy (GA1) and (GA2). Then 
(a)

$$
\begin{aligned}
& \left(D_{1} J_{r k}\left(g_{1}, g_{2}\right)-D_{2}^{\prime} J_{r k}\left(g_{1}, g_{2}\right), \xi\right)=0, \\
& \left(D_{2} J_{r k}\left(g_{1}, g_{2}\right)-D_{1}^{\prime} J_{r k}\left(g_{1}, g_{2}\right), \xi\right)=0
\end{aligned}
$$

for all $\xi \in \mathfrak{k}, 0 \leq k \leq r, 1 \leq r \leq n-1$.

(b) $\left(D_{1} \phi_{r k}\left(g_{1}, g_{2}\right)-D_{2}^{\prime} \phi_{r k}\left(g_{1}, g_{2}\right), \xi\right)=\left(D_{2} \phi_{r k}\left(g_{1}, g_{2}\right)-D_{1}^{\prime} \phi_{r k}\left(g_{1}, g_{2}\right), \xi\right)=0$ for all $\xi \in \mathfrak{k}, \quad 1 \leq k \leq r-1,2 \leq r \leq n-1$,

(c) for any $1 \leq r \leq n-1$,

$$
\begin{array}{ll}
\left(D_{1} \phi_{r 0}\left(g_{1}, g_{2}\right)-D_{2}^{\prime} \phi_{r 0}\left(g_{1}, g_{2}\right), \xi\right)=0 & \text { for all } \xi \in \mathfrak{k}, \\
\left(D_{2} \phi_{r 0}\left(g_{1}, g_{2}\right)-D_{1}^{\prime} \phi_{r 0}\left(g_{1}, g_{2}\right), \eta\right)=0 & \text { for all } \eta \in \widehat{\mathfrak{k}}=\operatorname{Lie}(\widehat{K}),
\end{array}
$$

(d) for any $1 \leq r \leq n-1$,

$$
\begin{array}{ll}
\left(D_{1} \phi_{r r}\left(g_{1}, g_{2}\right)-D_{2}^{\prime} \phi_{r r}\left(g_{1}, g_{2}\right), \xi\right)=0 & \text { for all } \xi \in \mathfrak{k}, \\
\left(D_{2} \phi_{r r}\left(g_{1}, g_{2}\right)-D_{1}^{\prime} \phi_{r r}\left(g_{1}, g_{2}\right), \eta\right)=0 & \text { for all } \eta \in \widehat{\mathfrak{k}} .
\end{array}
$$

To conclude this section, we now show that the set of $\left(g_{1}, g_{2}\right) \in \mathcal{U}$ which satisfies conditions (GA1) and (GA2) is an open, dense subset of $G^{2}$ of full measure. Indeed, it is clear that $\left\{\left(g_{1}, g_{2}\right) \in \mathcal{U} \mid\left(g_{1}, g_{2}\right)\right.$ satisfies (GA1) and (GA2) 2$\}$ is open and dense in $G^{2}$ and has full measure. To handle $(\mathrm{GA} 2)_{1}$, note that from the relationship between common zeros of homogeneous polynomials and resultant systems [VdW], it is enough to exhibit an element in $G^{2}$ with the property that the polynomials

$$
\tilde{J}\left(g_{1}, g_{2} ; u, h, z\right), \tilde{J}_{h}\left(g_{1}, g_{2} ; u, h, z\right), \tilde{J}_{z}\left(g_{1}, g_{2} ; u, h, z\right)
$$

do not have a common zero in $\mathbf{C}^{3} \backslash\{0\}$.

Proposition 3.20. There exists an element $\left(g_{1}^{\prime}, g_{2}^{\prime}\right) \in G^{2}$ such that 0 is a regular value of $\tilde{J}\left(g_{1}^{\prime}, g_{2}^{\prime} ; \cdot, \cdot, \cdot\right): \mathbf{C}^{3} \backslash\{0\} \rightarrow \mathbf{C}$.

Proof. Let

$$
\tilde{g}_{1}=I, \quad \tilde{g}_{2}=\left(\begin{array}{ccccc}
\gamma_{1} & 1 & & & \\
& \gamma_{2} & 1 & & \\
& & \ddots & \ddots & \\
& & 0 & & 1 \\
& & & & \gamma_{n}
\end{array}\right),
$$

where the $\gamma_{i}$ 's are distinct positive numbers. Consider the map

$$
\tilde{J}: N_{\left(\tilde{g_{1}}, \tilde{g_{2}}\right)} \times\left(\mathbf{C}^{3} \backslash\{0\}\right) \rightarrow \mathbf{C}
$$

defined in (2.10), where $N_{\left(\tilde{g_{1}}, \tilde{g_{2}}\right)}$, is a small neighborhood of $\left(\tilde{g_{1}}, \tilde{g_{2}}\right)$ to be specified in a moment. We would like to show that 0 is a regular value of $\tilde{J}$. For this purpose, it is enough to restrict ourselves to the annulus $\left\{(u, h, z) \in \mathbf{C}^{3} \mid 1 \leq\|(u, h, z)\| \leq 2\right\}$, and, for $N_{\left(\tilde{g_{1}}, \tilde{g_{2}}\right)}$ sufficiently small, all we need to do is to show that $\tilde{J}\left(\tilde{g_{1}}, \tilde{g_{2}} ; u, h, z\right)$, $\tilde{J}_{h}\left(\tilde{g_{1}}, \tilde{g_{2}} ; u, h, z\right), \tilde{J}_{z}\left(\tilde{g_{1}}, \tilde{g_{2}} ; u, h, z\right), \nabla_{1} \tilde{J}\left(\tilde{g_{1}}, \tilde{g_{2}} ; u, h, z\right)$ and $\nabla_{2} \tilde{J}\left(\tilde{g_{1}}, \tilde{g_{2}} ; u, h, z\right)$ have no common zeros in this annulus. Suppose the contrary, then from

$$
\nabla_{1} \tilde{J}\left(\tilde{g_{1}}, \tilde{g_{2}} ; u, h, z\right)=u\left(\operatorname{adj}\left(u \tilde{g_{2}} \tilde{g_{1}}-h \tilde{g}_{2}{\tilde{g_{2}}}^{T}-z\right)\right) g_{2},
$$

we have either $u=0$ or $\operatorname{adj}\left(u \tilde{g_{2}} \tilde{g_{1}}-h \tilde{g_{2}}{\tilde{g_{2}}}^{T}-z\right)=0$. If the latter is the case, then from

$$
\left.\left(\operatorname{adj}\left(u \tilde{g_{2}} \tilde{g_{1}}-h{\tilde{g_{2}}}_{\tilde{g}_{2}}^{T}-z\right)\right)_{1 n}=(-1)^{n+1}\left(u-h \gamma_{2}\right) \ldots\left(u-h \gamma_{n}\right)\right)
$$


and

$$
\left(\operatorname{adj}\left(u \tilde{g_{2}} \tilde{g}_{1}-h \tilde{g}_{2}{\tilde{g_{2}}}^{T}-z\right)\right)_{n 1}=\gamma_{2} \cdots \gamma_{n} h^{n-1}
$$

we conclude that $h=u=0$, and hence $z=0$ from $\tilde{J}\left(\tilde{g_{1}}, \tilde{g_{2}} ; u, h, z\right)=0$. As $(0,0,0)$ is not in the annulus, we conclude that $\operatorname{adj}\left(u \tilde{g_{2}} \tilde{g_{1}}-h{\tilde{g_{2}}}_{g_{2}}{ }^{T}-z\right)$ is never zero in the annulus. Hence we must have $u=0$. But from

$$
\nabla_{2} \tilde{J}\left(\tilde{g_{1}}, \tilde{g_{2}} ; 0, h, z\right)=-2 h \tilde{g}_{2}^{T} a d j\left(-h \tilde{g_{2}}{\tilde{g_{2}}}^{T}-z\right)
$$

it follows that $h=0$. Again, this implies $z=0$, which is not possible. Hence 0 is a regular value of $\tilde{J}: N_{\left(\tilde{g_{1}}, \tilde{g_{2}}\right)} \times\left(\mathbf{C}^{3} \backslash\{0\}\right) \rightarrow \mathbf{C}$ for $N_{\left(\tilde{g_{1}}, \tilde{g_{2}}\right)}$ sufficiently small, as desired. But then by transversality $[\mathrm{GG}], 0$ must be a regular value of $\tilde{J}\left(g_{1}^{\prime}, g_{2}^{\prime} ; \cdot, \cdot, \cdot\right)$ for some $\left(g_{1}^{\prime}, g_{2}^{\prime}\right) \in N_{\left(\tilde{g_{1}}, \tilde{g_{2}}\right)}$.

Remark 3.21. Note that (GA1) and (GA2) are not common to all elements which lie on a generic symplectic leaf of the twisted structure.

\section{PoISSON BRACKETS}

In this section, we establish the claim that the variables introduced in section 3 are essentially the action-angle variables.

To begin the computation of Poisson brackets, observe that with the notation introduced in Remark 2.7, the twisted structure in Theorem 2.5 can be expressed in the following three ways:

$$
\begin{aligned}
2\left\{\psi_{1}, \psi_{2}\right\}= & \left\langle D^{\prime} \psi_{1}, \pi_{2}\left(D \psi_{2}\right)\right\rangle-\left\langle D \psi_{1}, \pi_{2}\left(D^{\prime} \psi_{2}\right)\right\rangle \\
& +\left\langle D^{\prime} \psi_{2}-\pi_{2}\left(D \psi_{2}\right), \Pi_{\mathfrak{k}}\left(D^{\prime} \psi_{1}+\pi_{2}\left(D \psi_{1}\right)\right)\right\rangle \\
& +\left\langle D \psi_{1}-\pi_{2}\left(D^{\prime} \psi_{1}\right), \Pi_{\mathfrak{k}}\left(D \psi_{2}+\pi_{2}\left(D^{\prime} \psi_{2}\right)\right)\right\rangle, \\
2\left\{\psi_{1}, \psi_{2}\right\}= & \left\langle D \psi_{1}, \pi_{2}\left(D^{\prime} \psi_{2}\right)\right\rangle-\left\langle D^{\prime} \psi_{1}, \pi_{2}\left(D \psi_{2}\right)\right\rangle \\
& -\left\langle D^{\prime} \psi_{2}-\pi_{2}\left(D \psi_{2}\right), \Pi_{\mathfrak{l}}\left(D^{\prime} \psi_{1}+\pi_{2}\left(D \psi_{1}\right)\right)\right\rangle \\
& -\left\langle D \psi_{1}-\pi_{2}\left(D^{\prime} \psi_{1}\right), \Pi_{\mathfrak{l}}\left(D \psi_{2}+\pi_{2}\left(D^{\prime} \psi_{2}\right)\right)\right\rangle, \\
2\left\{\psi_{1}, \psi_{2}\right\}= & \left\langle D \psi_{1}-\pi_{2}\left(D^{\prime} \psi_{1}\right), \Pi_{\mathfrak{k}}\left(D \psi_{2}+\pi_{2}\left(D^{\prime} \psi_{2}\right)\right)\right\rangle \\
& -\left\langle D^{\prime} \psi_{2}-\pi_{2}\left(D \psi_{2}\right), \Pi_{\mathfrak{l}}\left(D^{\prime} \psi_{1}+\pi_{2}\left(D \psi_{1}\right)\right)\right\rangle,
\end{aligned}
$$

where the projection operators $\Pi_{\mathfrak{k}}$ and $\Pi_{\mathfrak{l}}$ have been extended to $\mathfrak{g} \oplus \mathfrak{g}$. If, for $h_{1}, h_{2} \in L$,

$$
F\left(h_{1} g_{1} h_{2}^{-1}, h_{2} g_{2} h_{1}^{-1}\right)=F\left(g_{1}, g_{2}\right),
$$

then by differentiation, we find that

$$
D_{1} F-D_{2}^{\prime} F, D_{2} F-D_{1}^{\prime} F \text { are strictly lower triangular matrices. }
$$

Similarly, if

$$
H\left(k_{1} g_{1} k_{2}^{-1}, k_{2} g_{2} k_{1}^{-1}\right)=H\left(g_{1}, g_{2}\right), \quad k_{1}, k_{2} \in K,
$$

then

$$
D_{1} H-D_{2}^{\prime} H, D_{2} H-D_{1}^{\prime} H \text { are symmetric matrices. }
$$


Therefore, if $\psi_{1}$ and $\psi_{2}$ both satisfy (4.3a), then from (4.3b) and (4.1a) we have (4.4)

$$
\begin{aligned}
2\left\{\psi_{1}, \psi_{2}\right\} & =\left\langle D^{\prime} \psi_{1}, \pi_{2}\left(D \psi_{2}\right)\right\rangle-\left\langle D \psi_{1}, \pi_{2}\left(D^{\prime} \psi_{2}\right)\right\rangle \\
& =\left(D_{1}^{\prime} \psi_{1}, D_{2} \psi_{2}\right)+\left(D_{2}^{\prime} \psi_{1}, D_{1} \psi_{2}\right)-\left(D_{1} \psi_{1}, D_{2}^{\prime} \psi_{2}\right)-\left(D_{2} \psi_{1}, D_{1}^{\prime} \psi_{2}\right) .
\end{aligned}
$$

On the other hand, if $\psi_{1}$ satisfies (4.3a), while $\psi_{2}$ satisfies (4.2a), then from (4.2b), (4.3b) and (4.1c), it follows that

$$
\left\{\psi_{1}, \psi_{2}\right\}=0
$$

In the following, we shall evaluate the Poisson brackets at a point $\left(g_{1}, g_{2}\right) \in \mathcal{U}$ satisfying (GA1) and (GA2).

Proposition 4.6. (a) $\left\{\lambda_{r k}, J_{r^{\prime} k^{\prime}}\right\}\left(g_{1}, g_{2}\right)=0, k \geq 1, k^{\prime} \geq 0$,

(b) $\left\{\lambda_{r k}, \phi_{r^{\prime} k^{\prime}}\right\}\left(g_{1}, g_{2}\right)=0,2 \leq r \leq n-k, 1 \leq k \leq n-1,1 \leq k^{\prime} \leq r^{\prime}-1,2 \leq$ $r^{\prime} \leq n-1$

(c) $\left\{\theta_{r k}, J_{r^{\prime} k^{\prime}}\right\}\left(g_{1}, g_{2}\right)=0,2 \leq r \leq n-k, 1 \leq k \leq n-1, k^{\prime} \geq 0$,

(d) $\left\{\theta_{r k}, \phi_{r^{\prime} k^{\prime}}\right\}\left(g_{1}, g_{2}\right)=0,2 \leq r \leq n-k, 1 \leq k \leq n-1,1 \leq k^{\prime} \leq r^{\prime}-1,2 \leq$ $r^{\prime} \leq n-1$.

Proof. This follows from Proposition 3.18 (a), (b), the invariance properties of $\lambda_{r k}, \theta_{r k}[\mathrm{~L} 3]$, and (4.5) above.

Proposition 4.7 [L3]. (a) $\left\{\lambda_{r k}, \lambda_{r^{\prime} k^{\prime}}\right\}\left(g_{1}, g_{2}\right)=0,2 \leq r \leq n-k, 2 \leq r^{\prime} \leq$ $n-k^{\prime}, 1 \leq k, k^{\prime} \leq n-1$,

(b) $\left\{\theta_{r k}, \lambda_{r^{\prime} k^{\prime}}\right\}\left(g_{1}, g_{2}\right)=\delta_{k k^{\prime}} \delta_{r r^{\prime}}, 2 \leq r \leq n-k, 1 \leq k \leq n-1,2 \leq r^{\prime} \leq$ $n-k^{\prime}, 1 \leq k^{\prime} \leq n-1$,

(c) $\left\{\theta_{r k}, \theta_{r^{\prime} k^{\prime}}\right\}\left(g_{1}, g_{2}\right)=0,2 \leq r \leq n-k, 2 \leq r^{\prime} \leq n-k^{\prime}, 1 \leq k, k^{\prime} \leq n-1$.

Proposition 4.8. $\left\{J_{r k}, J_{r^{\prime} k^{\prime}}\right\}\left(g_{1}, g_{2}\right)=0, k, k^{\prime} \geq 0$.

Proof. Let $H_{h, z}\left(g_{1}, g_{2}\right)=\log \operatorname{det}\left(g_{2} g_{1}-h g_{2} g_{2}^{T}-z\right), \quad[1: h: z] \notin \mathcal{C}\left(g_{1}, g_{2}\right)$. From Proposition 3.18(a) and (4.4), we have

$$
\begin{aligned}
2\left\{H_{h, z}\right. & \left., H_{h^{\prime} z^{\prime}}\right\}\left(g_{1}, g_{2}\right) \\
= & \left(D_{1}^{\prime} H_{h, z}, D_{2} H_{h^{\prime}, z^{\prime}}\right)+\left(D_{2}^{\prime} H_{h, z}, D_{1} H_{h^{\prime}, z^{\prime}}\right) \\
& -\left(D_{1} H_{h, z}, D_{2}^{\prime} H_{h^{\prime}, z^{\prime}}\right)-\left(D_{2} H_{h, z}, D_{1}^{\prime} H_{h^{\prime}, z^{\prime}}\right) \\
= & \left(D_{1}^{\prime} H_{h, z}-D_{2} H_{h, z}, D_{2} H_{h^{\prime}, z^{\prime}}+D_{1}^{\prime} H_{h^{\prime}, z^{\prime}}\right) \\
& -\left(D_{1} H_{h, z}-D_{2}^{\prime} H_{h, z}, D_{2}^{\prime} H_{h^{\prime}, z^{\prime}}+D_{1} H_{h^{\prime}, z^{\prime}}\right) .
\end{aligned}
$$

Now, by a straightforward computation, we get $D_{1} H_{h, z}\left(g_{1}, g_{2}\right)=g_{1}\left(M_{h}-z\right)^{-1} g_{2}$, and $D_{2}^{\prime} H_{h, z}\left(g_{1}, g_{2}\right)=I+z g_{2}^{-1}\left(M_{h}-z\right)^{-1} g_{2}-h g_{2}^{T}\left(M_{h}-z\right)^{-T} g_{2}$. Hence,

$$
D_{1} H_{h, z}\left(g_{1}, g_{2}\right)-D_{2}^{\prime} H_{h, z}\left(g_{1}, g_{2}\right)=h g_{2}^{T}\left(\left(M_{h}-z\right)^{-1}+\left(M_{h}-z\right)^{-T}\right) g_{2},
$$

while

$$
\begin{aligned}
& D_{1} H_{h^{\prime}, z^{\prime}}\left(g_{1}, g_{2}\right)+D_{2}^{\prime} H_{h^{\prime}, z^{\prime}}\left(g_{1}, g_{2}\right) \\
& \quad=2 I+2 z^{\prime} g_{2}^{-1}\left(M_{h^{\prime}}-z^{\prime}\right)^{-1} g_{2}+h^{\prime} g_{2}^{T}\left(\left(M_{h^{\prime}}-z^{\prime}\right)^{-1}-\left(M_{h^{\prime}}-z^{\prime}\right)^{-T}\right) g_{2} .
\end{aligned}
$$

Similarly,

$$
D_{1}^{\prime} H_{h, z}\left(g_{1}, g_{2}\right)-D_{2} H_{h, z}\left(g_{1}, g_{2}\right)=h\left(\left(M_{h}-z\right)^{-1} g_{2} g_{2}^{T}+g_{2} g_{2}^{T}\left(M_{h}-z\right)^{-T}\right),
$$




$$
\begin{aligned}
& D_{2} H_{h^{\prime}, z^{\prime}}\left(g_{1}, g_{2}\right)+D_{1}^{\prime} H_{h^{\prime}, z^{\prime}}\left(g_{1}, g_{2}\right) \\
& \quad=2 I+2 z^{\prime}\left(M_{h^{\prime}}-z^{\prime}\right)^{-1}+h^{\prime}\left(\left(M_{h^{\prime}}-z^{\prime}\right)^{-1} g_{2} g_{2}^{T}-g_{2} g_{2}^{T}\left(M_{h^{\prime}}-z^{\prime}\right)^{-T}\right) .
\end{aligned}
$$

Therefore, when we substitute these expressions into (4.9) and simplify, we obtain

$$
\begin{aligned}
2\left\{H_{h, z},\right. & \left.H_{h^{\prime}, z^{\prime}}\right\}\left(g_{1}, g_{2}\right) \\
= & h\left(\left(M_{h}-z\right)^{-1} g_{2} g_{2}^{T}+g_{2} g_{2}^{T}\left(M_{h}-z\right)^{-T}, 2 I+2 z^{\prime}\left(M_{h^{\prime}}-z^{\prime}\right)^{-1}\right) \\
& -h\left(g_{2}^{T}\left(M_{h}-z\right)^{-1} g_{2}+g_{2}^{T}\left(M_{h}-z\right)^{-T} g_{2}, 2 I+2 z^{\prime} g_{2}^{-1}\left(M_{h^{\prime}}-z^{\prime}\right)^{-1} g_{2}\right) \\
= & 2 h z^{\prime} \operatorname{tr}\left(\left[\left(M_{h}-z\right)^{-1}, g_{2} g_{2}^{T}\right]\left(M_{h^{\prime}}-z^{\prime}\right)^{-1}\right)
\end{aligned}
$$

But $g_{2} g_{2}^{T}=\left(M_{h}-M_{h^{\prime}}\right) /\left(h^{\prime}-h\right)$; hence it follows that

$$
\begin{aligned}
2\left\{H_{h, z},\right. & \left.H_{h^{\prime}, z^{\prime}}\right\}\left(g_{1}, g_{2}\right) \\
= & \frac{2 h z^{\prime}}{h^{\prime}-h} \operatorname{tr}\left(\left[\left(M_{h}-z\right)^{-1}, M_{h}\right]\left(M_{h^{\prime}}-z^{\prime}\right)^{-1}\right) \\
& -\frac{2 h z^{\prime}}{h^{\prime}-h} \operatorname{tr}\left(\left[M_{h^{\prime}},\left(M_{h^{\prime}}-z^{\prime}\right)^{-1}\right]\left(M_{h}-z\right)^{-1}\right) \\
= & 0 .
\end{aligned}
$$

Proposition 4.9. (a) $\left\{\phi_{r k}, J_{r^{\prime} k^{\prime}}\right\}\left(g_{1}, g_{2}\right)=-\delta_{r r^{\prime}} \delta_{k k^{\prime}}, 1 \leq k \leq r-1,2 \leq r \leq$ $n-k, k^{\prime} \geq 0$,

(b) $\left\{\phi_{r 0}, J_{r^{\prime} k^{\prime}}\right\}\left(g_{1}, g_{2}\right)=-\delta_{k^{\prime} 0} \delta_{r r^{\prime}}, 1 \leq r \leq n-1, \quad k^{\prime} \geq 0$.

Proof. (a) As in Proposition 4.8, consider

$$
H_{h_{0}, z_{0}}\left(g_{1}, g_{2}\right)=\log \operatorname{det}\left(g_{2} g_{1}-h_{0} g_{2} g_{2}^{T}-z_{0}\right),\left[1: h_{0}: z_{0}\right] \notin \mathcal{C}\left(g_{1}, g_{2}\right) .
$$

From (4.1c) and (4.3b), the Hamilton equation generated by $H_{h_{0}, z_{0}}$ is given by

$$
\begin{aligned}
& \dot{g}_{1}=\frac{1}{2} g_{1}\left(\Pi_{\mathfrak{l}}\left(D_{1}^{\prime} H_{h_{0}, z_{0}}+D_{2} H_{h_{0}, z_{0}}\right)\right)-\frac{1}{2}\left(\Pi_{\mathfrak{l}}\left(D_{1} H_{h_{0}, z_{0}}+D_{2}^{\prime} H_{h_{0}, z_{0}}\right)\right) g_{1}, \\
& \dot{g}_{2}=\frac{1}{2} g_{2}\left(\Pi_{\mathfrak{l}}\left(D_{1} H_{h_{0}, z_{0}}+D_{2}^{\prime} H_{h_{0}, z_{0}}\right)\right)-\frac{1}{2}\left(\Pi_{\mathfrak{l}}\left(D_{1}^{\prime} H_{h_{0}, z_{0}}+D_{2} H_{h_{0}, z_{0}}\right)\right) g_{2} .
\end{aligned}
$$

This induces a flow on $M_{h}=M_{h}\left(g_{1}, g_{2}\right)$, and a direct computation gives an equation in Lax pair form:

$$
\dot{M}_{h}=\left[M_{h}, B_{h}\right]
$$

where

$$
B_{h}=\frac{1}{2} \Pi_{\mathfrak{l}}\left(D_{1}^{\prime} H_{h_{0}, z_{0}}+D_{2} H_{h_{0}, z_{0}}\right)+\frac{h z_{0}}{h_{0}-h}\left(M_{h_{0}}-z_{0}\right)^{-1} .
$$

Let $P_{i}=\left[1: h_{0}: z_{i}\left(g_{1}, g_{2}, h_{0}\right)\right] \in \mathcal{C}\left(g_{1}, g_{2}\right), \quad i=1, \ldots, n$. To compute $\left\{\phi_{r k}, H_{h_{0}, z_{0}}\right\}\left(g_{1}, g_{2}\right)$, it is enough to evaluate it on the open dense set of $\left(g_{1}, g_{2}\right) \in \mathcal{U}$ satisfying

(i) $\operatorname{supp} D\left(g_{1}, g_{2}\right) \cap\left\{(h, z) \mid J_{z}\left(g_{1}, g_{2} ; h, z\right)=0\right\}=\phi$,

(ii) $\operatorname{supp} D\left(g_{1}, g_{2}\right) \subset \mathcal{C}_{a}\left(g_{1}, g_{2}\right)$,

(iii) supp $D\left(g_{1}, g_{2}\right) \cap\left\{(h, z) \mid \widehat{J}_{z}\left(g_{1}, g_{2} ; h, z\right)=0\right\}=\phi$ where $\widehat{J}_{z}\left(g_{1}, g_{2} ; h, z\right)=$ $\operatorname{det}\left(M_{h, z}\left(g_{1}, g_{2}\right)\right)^{\wedge}$ (for a matrix $M, \widehat{M}$ is the submatrix obtained from $M$ by deleting the first row and column),

(iv) $\operatorname{supp} D\left(g_{1}, g_{2}\right) \cap\left\{P_{i}\right\}_{i=1}^{n}=\phi$,

(v) $\left\{P_{i}\right\}_{i=1}^{n} \cap\left\{(h, z) \mid J_{z}\left(g_{1}, g_{2} ; h, z\right)=0\right\}=\phi$. 
So suppose $\left(g_{1}, g_{2}\right)$ satisfies (i)-(v). By (i), (ii) and (iii), supp $D\left(g_{1}, g_{2}\right)$ is in the affine part of $\mathcal{C}\left(g_{1}, g_{2}\right)$ away from the branch points, so in the neighborhood of each of the points $D_{i}=\left[1: h_{i}: z_{i}\right] \in \operatorname{supp} D\left(g_{1}, g_{2}\right)$, we can use $h$ as local coordinate. Similarly, by (v), we can use $h$ as local coordinate in the neighborhood of each of the points $P_{i}$. Let $\left(g_{1}(t), g_{2}(t)\right)$ denote the solution of the Hamilton equation generated by $H_{h_{0}, z_{0}}$ with initial conditions $g_{1}(t=0)=g_{1}, g_{2}(t=0)=g_{2}$. Then

$$
\left\{\phi_{r k}, H_{h_{0}, z_{0}}\right\}\left(g_{1}, g_{2}\right)=\left.\frac{d}{d t}\right|_{t=0} \phi_{r k}\left(g_{1}(t), g_{2}(t)\right)=\left.\sum_{i} \frac{h_{i}^{k-1} z_{i}^{n-r-1}}{J_{z}\left(h_{i}, z_{i}\right)} \frac{d h_{i}}{d t}\right|_{t=0}
$$

Now let $f(h, t)$ denote the representative of the kernel map $f_{t}$ of $M\left(g_{1}(t), g_{2}(t), p\right)$ near $h_{i}$. Then from the defining relation $\left(e_{1}, f\left(h_{i}, t\right)\right)=0$, we obtain

$$
\left.\frac{d h_{i}}{d t}\right|_{t=0}=\frac{h_{i} z_{0}}{h_{0}-h_{i}} \frac{\left(e_{1},\left(M_{h_{0}}-z_{0}\right)^{-1} f\left(h_{i}\right)\right)}{\left(e_{1}, \frac{\partial f}{\partial h}\left(h_{i}, 0\right)\right)},
$$

where we have used the explicit form of $B_{h}$. Thus,

$$
\begin{aligned}
& \left\{\phi_{r k}, H_{h_{0}, z_{0}}\right\}\left(g_{1}, g_{2}\right) \\
& \quad=\sum_{i} \frac{h_{i}^{k} z_{i}^{n-r-1} z_{0}}{\left(h_{0}-h_{i}\right) J_{z}\left(h_{i}, z_{i}\right)} \cdot \frac{\left(e_{1},\left(M_{h_{0}}-z_{0}\right)^{-1} f\left(h_{i}\right)\right)}{\left(e_{1}, \frac{\partial f}{\partial h}\left(h_{i}, 0\right)\right)} .
\end{aligned}
$$

To evaluate this, we apply the residue theorem to the meromorphic 1-form

$$
\frac{\left(e_{1},\left(M_{h_{0}}-z_{0}\right)^{-1} f(h)\right) h z_{0}}{\left(h_{0}-h\right)\left(e_{1}, f(h)\right)} \omega_{r+1, k-1}
$$

which has poles precisely at the points $P_{i}, i=1, \ldots, n$, and $\operatorname{supp} D\left(g_{1}, g_{2}\right)$. This gives

$$
\begin{aligned}
\left\{\phi_{r k}, H_{h_{0}, z_{0}}\right\}\left(g_{1}, g_{2}\right) & =-\sum_{i} \operatorname{res}_{P_{i}} \frac{\left(e_{1},\left(M_{h_{0}}-z_{0}\right)^{-1} f(h)\right) h z_{0}}{\left(h_{0}-h\right)\left(e_{1}, f(h)\right)} \omega_{r+1, k-1} \\
& =h_{0}^{k} z_{0} \sum_{i} \frac{z_{i}\left(h_{0}\right)^{n-r-1}}{\left(z_{i}\left(h_{0}\right)-z_{0}\right) J_{z}\left(h_{0}, z_{i}\left(h_{0}\right)\right)} \\
& =h_{0}^{k} z_{0}\left[\lim _{R \rightarrow \infty} \oint_{|z|=R} \frac{z^{n-r-1}}{\left(z-z_{0}\right) J\left(h_{0}, z\right)} \frac{d z}{2 \pi \sqrt{-1}}-\frac{z_{0}^{n-r-1}}{J\left(h_{0}, z_{0}\right)}\right] \\
& =\frac{-h_{0}^{k} z_{0}^{n-r}}{J\left(h_{0}, z_{0}\right)}, \quad \text { as } r \geq 2 .
\end{aligned}
$$

As

$$
\left\{\phi_{r k}, H_{h_{0}, z_{0}}\right\}=\frac{1}{J\left(h_{0}, z_{0}\right)} \sum_{k^{\prime}, r^{\prime}}\left\{\phi_{r k}, J_{r^{\prime} k^{\prime}}\right\} h_{0}^{k^{\prime}} z_{0}^{n-r^{\prime}},
$$

the above computation shows that $\left\{\phi_{r k}, J_{r^{\prime}, k^{\prime}}\right\}\left(g_{1}, g_{2}\right)=-\delta_{r r^{\prime}} \delta_{k k^{\prime}}$.

(b) For $\phi_{r 0}$, the same argument shows that

$$
\left\{\phi_{r 0}, H_{h_{0}, z_{0}}\right\}\left(g_{1}, g_{2}\right)=z_{0} \sum_{i} \frac{z_{i}\left(h_{0}\right)^{n-r-1}}{\left(z_{i}\left(h_{0}\right)-z_{0}\right) J_{z}\left(h_{0}, z_{i}\left(h_{0}\right)\right)}=\frac{-z_{0}^{n-r}}{J\left(h_{0}, z_{0}\right)},
$$

which implies $\left\{\phi_{r 0}, J_{r^{\prime} k^{\prime}}\right\}\left(g_{1}, g_{2}\right)=-\delta_{k^{\prime} 0} \delta_{r r^{\prime}}$.

Proposition 4.11. $\left\{\phi_{r r}, J_{r^{\prime} k^{\prime}}\right\}\left(g_{1}, g_{2}\right)=-\delta_{r r^{\prime}} \delta_{k^{\prime} r^{\prime}}, 1 \leq r \leq n-1, k^{\prime} \geq 0$. 
Proof. We shall use the notation introduced in the proof of Proposition 4.9. To compute the bracket $\left\{\phi_{r r}, H_{h_{0}, z_{0}}\right\}\left(g_{1}, g_{2}\right)$, it is enough to evaluate it on the open dense set of $\left(g_{1}, g_{2}\right) \in \mathcal{U}$ satisfying (i)-(iv) in the proof of Proposition 4.9 plus analogous conditions for $D^{T}\left(g_{1}, g_{2}\right)$. So let $\left(g_{1}, g_{2}\right)$ satisfy these conditions. By assumption, $D^{T}\left(g_{1}, g_{2}\right)$ consists of $\frac{1}{2} n(n-1)$ distinct points $D_{i}=\left[1: h_{i}^{\prime}: z_{i}^{\prime}\right]$. Therefore,

$$
\left\{\int_{D_{0}}^{D^{T}} \omega_{r+1, r-1}, H_{h_{0}, z_{0}}\right\}\left(g_{1}, g_{2}\right)=\left.\sum_{i} \frac{{h_{i}{ }^{r-1} z_{i}{ }^{n-r-1}}^{J_{z}\left(h_{i}^{\prime}, z_{i}^{\prime}\right)}}{d t}\right|_{t=0} .
$$

Let $f^{T}(h, t)$ be a representative of the kernel map $f_{t}^{T}$ of $\left(M\left(g_{1}(t), g_{2}(t) ; p\right)\right)^{T}$ near $h_{i}^{\prime}$; then

$$
\begin{aligned}
\left.\frac{d h_{i}^{\prime}}{d t}\right|_{t=0}= & -\left(e_{1}, \frac{\partial f^{T}}{\partial t}\left(h_{i}^{\prime}, 0\right)\right) /\left(e_{1}, \frac{\partial f^{T}}{\partial h}\left(h_{i}^{\prime}, 0\right)\right) \\
= & -\left(e_{1}, B_{h_{i}^{\prime}}^{T} f^{T}\left(h_{i}^{\prime}\right)\right) /\left(e_{1}, \frac{\partial f^{T}}{\partial h}\left(h_{i}^{\prime}, 0\right)\right) \\
= & -z_{0}\left(e_{1},\left(M_{h_{0}}-z_{0}\right)^{-1} f^{T}\left(h_{i}^{\prime}\right)\right) /\left(e_{1}, \frac{\partial f^{T}}{\partial h}\left(h_{i}^{\prime}\right)\right) \\
& -\frac{h_{0} z_{0}}{h_{0}-h_{i}^{\prime}}\left(e_{1},\left(M_{h_{0}}-z_{0}\right)^{-T} f^{T}\left(h_{i}^{\prime}\right)\right) /\left(e_{1}, \frac{\partial f^{T}}{\partial h}\left(h_{i}^{\prime}\right)\right)
\end{aligned}
$$

(from (4.10)). Hence,

$$
\begin{aligned}
\left\{\int_{D_{0}}^{D^{T}}\right. & \left.\omega_{r+1, r-1}, H_{h_{0}, z_{0}}\right\}\left(g_{1}, g_{2}\right) \\
= & -z_{0} \sum_{i} \frac{h_{i}^{\prime}{ }^{r-1} z_{i}^{\prime}{ }^{n-r-1}}{J_{z}\left(h_{i}^{\prime}, z_{i}^{\prime}\right)} \frac{\left(e_{1},\left(M_{h_{0}}-z_{0}\right)^{-1} f^{T}\left(h_{i}^{\prime}\right)\right)}{\left(e_{1}, \frac{\partial f^{T}}{\partial h}\left(h_{i}^{\prime}\right)\right)} \\
& \quad-h_{0} z_{0} \sum_{i} \frac{h_{i}^{\prime}{ }^{r-1} z_{i}^{\prime}{ }^{n-r-1}}{J_{z}\left(h_{i}^{\prime}, z_{i}^{\prime}\right)} \frac{\left(e_{1},\left(M_{h_{0}}-z_{0}\right)^{-T} f^{T}\left(h_{i}^{\prime}\right)\right)}{\left(h_{0}-h_{i}^{\prime}\right)\left(e_{1}, \frac{\partial f^{T}}{\partial h}\left(h_{i}^{\prime}\right)\right)} .
\end{aligned}
$$

Applying the residue theorem to the meromorphic 1-form

$$
\frac{z_{0}\left(e_{1},\left(M_{h_{0}}-z_{0}\right)^{-1} f^{T}(h)\right)}{\left(e_{1}, f^{T}(h)\right)} \omega_{r+1, r-1},
$$

which has poles at supp $D^{T}\left(g_{1}, g_{2}\right)$ and $\left\{P_{-}^{i}\right\}_{i=1}^{n}$, we find that

first term in $(\dagger)$

$$
\begin{aligned}
& =-\sum_{i} \operatorname{res}_{D_{i}^{\prime}} \frac{z_{0}\left(e_{1},\left(M_{h_{0}}-z_{0}\right)^{-1} f^{T}(h)\right)}{\left(e_{1}, f^{T}(h)\right)} \omega_{r+1, r-1} \\
& =\sum_{j} \operatorname{res}_{P_{-}^{j}} \frac{z_{0}\left(e_{1},\left(M_{h_{0}}-z_{0}\right)^{-1} f^{T}(p)\right)}{\left(e_{1}, f^{T}(p)\right)} \omega_{r+1, r-1} \\
& =(-1)^{n-r-1} z_{0} \sum_{j} \frac{\left(e_{1},\left(M_{h_{0}}-z_{0}\right)^{-1} f\left(P_{-}^{j}\right)\right)}{\left(e_{1}, f\left(P_{-}^{i}\right)\right)} \frac{\mu_{j}^{n-r-1}}{\prod_{i \neq j}\left(\mu_{j}-\mu_{i}\right)} .
\end{aligned}
$$


Similarly, by applying the residue theorem to the meromorphic 1-form

$$
\frac{h_{0} z_{0}}{h_{0}-h} \frac{\left(e_{1},\left(M_{h_{0}}-z_{0}\right)^{-T} f^{T}(h)\right)}{\left(e_{1}, f^{T}(h)\right)} \omega_{r+1, r-1},
$$

which has poles at $D^{T}\left(g_{1}, g_{2}\right)$ and $\left\{P_{j}\right\}_{j=1}^{n}$, we find that

second term in $(\dagger)$

$$
\begin{aligned}
& =\sum_{j} \operatorname{res}_{P_{j}} \frac{h_{0} z_{0}}{h_{0}-h} \frac{\left(e_{1},\left(M_{h_{0}}-z_{0}\right)^{-T} f^{T}(h)\right)}{\left(e_{1}, f^{T}(h)\right)} \omega_{r+1, r-1} \\
& =-\sum_{j} \frac{h_{0}^{r} z_{0} z_{j}\left(h_{0}\right)^{n-r-1}}{\left(z_{j}\left(h_{0}\right)-z_{0}\right) J_{z}\left(h_{0}, z_{j}\left(h_{0}\right)\right)} \\
& =\frac{h_{0}^{r} z_{0}^{n-r}}{J\left(h_{0}, z_{0}\right)} .
\end{aligned}
$$

Putting things together, we get

$$
\begin{aligned}
& \left\{\int_{D_{0}}^{D^{T}} \omega_{r+1, r-1}, H_{h_{0}, z_{0}}\right\}\left(g_{1}, g_{2}\right) \\
& \quad=\frac{h_{0}^{r} z_{0}^{n-r}}{J\left(h_{0}, z_{0}\right)}+(-1)^{n-r-1} z_{0} \sum_{j} \frac{\left(e_{1},\left(M_{h_{0}}-z_{0}\right)^{-1} f\left(P_{-j}^{j}\right)\right)}{\left(e_{1}, f\left(P_{-}^{j}\right)\right)} \frac{\mu_{j}^{n-r-1}}{\prod_{(i \neq j)}\left(\mu_{j}-\mu_{i}\right)} .
\end{aligned}
$$

In a similar way, we find that

$$
\begin{aligned}
& \left\{\int_{D_{0}}^{D} \omega_{r+1, r-1}, H_{h_{0}, z_{0}}\right\}\left(g_{1}, g_{2}\right) \\
& \quad=\frac{-h_{0}^{r} z_{0}^{n-r}}{J\left(h_{0}, z_{0}\right)}+(-1)^{n-r-1} z_{0} \sum_{j} \frac{\left(e_{1},\left(M_{h_{0}}-z_{0}\right)^{-1} f\left(P_{-}^{j}\right)\right)}{\left(e_{1}, f\left(P_{-}^{j}\right)\right)} \frac{\mu_{j}^{n-r-1}}{\prod_{i \neq j}\left(\mu_{j}-\mu_{i}\right)},
\end{aligned}
$$

and hence

$$
\left\{\int_{D^{T}}^{D} \omega_{r+1, r-1}, H_{h_{0}, z_{0}}\right\}\left(g_{1}, g_{2}\right)=\frac{-2 h_{0}^{r} z_{0}^{n-r}}{J\left(h_{0}, z_{0}\right)}
$$

Proposition 4.12. (a) $\left\{\phi_{r 0}, \lambda_{r^{\prime} k^{\prime}}\right\}\left(g_{1}, g_{2}\right)=0, \quad 1 \leq r \leq n-1,2 \leq r^{\prime} \leq$ $n-k^{\prime}, 1 \leq k^{\prime} \leq n-1$,

(b) $\left\{\phi_{r 0}, \theta_{r^{\prime} k^{\prime}}\right\}\left(g_{1}, g_{2}\right)=0,1 \leq r \leq n-1,2 \leq r^{\prime} \leq n-k^{\prime}, 1 \leq k^{\prime} \leq n-1$.

Proof. Let $F=\lambda_{r^{\prime} k^{\prime}}$ or $\theta_{r^{\prime} k^{\prime}}$, then by the invariance properties of these variables [L3] and (4.1c), we have

$$
\begin{aligned}
2\left\{\phi_{r 0}, F\right\}\left(g_{1}, g_{2}\right)=\langle & \left.D \phi_{r 0}-\pi_{2}\left(D^{\prime} \phi_{r 0}\right), \Pi_{\mathfrak{k}}\left(D F+\pi_{2}\left(D^{\prime} F\right)\right)\right\rangle \\
= & \left(D_{1} \phi_{r 0}-D_{2}^{\prime} \phi_{r 0}, \Pi_{\mathfrak{k}}\left(D_{1} F+D_{2}^{\prime} F\right)\right) \\
& +\left(D_{2} \phi_{r 0}-D_{1}^{\prime} \phi_{r 0}, \Pi_{\mathfrak{k}}\left(D_{2} F+D_{1}^{\prime} F\right)\right) .
\end{aligned}
$$

Now, from Corollary 3.19, $\left(D_{1} \phi_{r 0}-D_{2}^{\prime} \phi_{r 0}, \xi\right)=0, \quad\left(D_{2} \phi_{r 0}-D_{1}^{\prime} \phi_{r 0}, \eta\right)=0$ for all $\xi \in \mathfrak{k}$, and for all $\eta \in \widehat{\mathfrak{k}}$. This immediately implies the vanishing of the first term in $(*)$. For the second term, note that $\Pi_{\mathfrak{k}}\left(D_{2} F+D_{1}^{\prime} F\right)=2 \Pi_{\mathfrak{k}}\left(D_{1}^{\prime} F\right)$ as 
$D_{2} F-D_{1}^{\prime} F$ is strictly lower triangular. Then, by the definition of $\lambda_{r k}$ and the proof of Proposition 4.14 in [L3], the first rows of $D_{1}^{\prime} \lambda_{r k}$ and $D_{1}^{\prime}\left(m_{r k} / m_{1 k}\right)$ are equal to zero. Consequently, $\Pi_{\mathfrak{k}} D_{1}^{\prime} \lambda_{r k}, \Pi_{\mathfrak{k}} D_{1}^{\prime}\left(m_{r k} / m_{1 k}\right) \in \widehat{\mathfrak{k}}$, and this implies that the second term in $(*)$ is also zero.

In a similar fashion, we obtain

Proposition 4.13. (a) $\left\{\phi_{r r}, \lambda_{r^{\prime} k^{\prime}}\right\}\left(g_{1}, g_{2}\right)=0,1 \leq r \leq n-1,2 \leq r^{\prime} \leq$ $n-k^{\prime}, 1 \leq k^{\prime} \leq n-1$,

(b) $\left\{\phi_{r r}, \theta_{r^{\prime} k^{\prime}}\right\}\left(g_{1}, g_{2}\right)=0,1 \leq r \leq n-1,2 \leq r^{\prime} \leq n-k^{\prime}, 1 \leq k^{\prime} \leq n-1$.

Remark 4.14. A calculation similar to that in the proof of Proposition 4.9 (a) yields

$$
\left\{\int_{D_{0}}^{D^{T}} \omega_{r+1, k-1}, J_{r^{\prime} k^{\prime}}\right\}=\delta_{r r^{\prime}} \delta_{k k^{\prime}}
$$

$1 \leq k \leq r-1,2 \leq r \leq n-k, k^{\prime} \geq 0$. However,

$$
\left\{\int_{D_{0}}^{D^{T}} \omega_{r+1,-1}, J_{r^{\prime} k^{\prime}}\right\} \neq-\left\{\int_{D_{0}}^{D} \omega_{r+1,-1}, J_{r^{\prime} k^{\prime}}\right\} .
$$

Corollary 4.15. The lattice Lax systems (1.8) are completely integrable on generic symplectic leaves of the twisted structure.

Corollary 4.16. Let $\left(g_{1}^{\circ}, g_{2}^{\circ}\right) \in \mathcal{U}$ satisfy (GA1) and (GA2). Then the level set of integrals

$$
\begin{aligned}
& I\left(g_{1}^{\circ}, g_{2}^{\circ}\right) \\
& \quad=\left\{\left(g_{1}, g_{2}\right) \in \mathcal{L}_{\left(g_{1}^{\circ}, g_{2}^{\circ}\right)} \mid \lambda_{r k}\left(g_{1}, g_{2}\right)=\lambda_{r k}\left(g_{1}^{\circ}, g_{2}^{\circ}\right), 2 \leq r \leq n-k, 1 \leq k \leq n-1,\right. \\
& \left.J_{r^{\prime} k^{\prime}}\left(g_{1}, g_{2}\right)=J_{r^{\prime} k^{\prime}}\left(g_{1}^{\circ}, g_{2}^{\circ}\right), 0 \leq k^{\prime} \leq r^{\prime}, 1 \leq r^{\prime} \leq n-1\right\}
\end{aligned}
$$

is a smooth manifold of dimension $n(n-1)$.

Corollary 4.17 (Local uniqueness). Let $\left(g_{1}^{\circ}, g_{2}^{\circ}\right) \in \mathcal{U}$ satisfy (GA1) and (GA2). Then the variables $\lambda_{r k}, \theta_{r k}, J_{r^{\prime} k^{\prime}}, \phi_{r^{\prime} k^{\prime}}, \phi_{r^{\prime} 0}, \phi_{r^{\prime} r^{\prime}}$ provide a diffeomorphism from a complex neighborhood of $\left(g_{1}^{\circ}, g_{2}^{\circ}\right)$ in the complexification of $\mathcal{L}_{\left(g_{1}^{\circ}, g_{2}^{\circ}\right)}$ onto an open set in $\mathbf{C}^{2 n(n-1)}$.

Let $I\left(g_{1}^{\circ}, g_{2}^{\circ}\right)$ be the level set of integrals in Corollary 4.16. If $\left(g_{1}, g_{2}\right) \in I\left(g_{1}^{\circ}, g_{2}^{\circ}\right)$, then $\sigma\left(g_{1}, g_{2}^{T}\right)=\sigma\left(g_{1}^{\circ}, g_{2}^{\circ T}\right)$ and $J\left(g_{1}, g_{2} ; h, z\right)=J\left(g_{1}^{\circ}, g_{2}^{\circ} ; h, z\right)$, so that $\left(g_{1}, g_{2}\right)$ also satisfies (GA1) and (GA2). Therefore, we can define the linearization map

$$
\begin{aligned}
\mathbf{L}: I\left(g_{1}^{\circ}, g_{2}^{\circ}\right) \ni\left(g_{1}, g_{2}\right) \mapsto & \left(\theta_{r k}\left(g_{1}, g_{2}\right), 2 \leq r \leq n-k, 1 \leq k \leq n-1 ;\right. \\
& \phi_{r^{\prime} k^{\prime}}\left(g_{1}, g_{2}\right), 1 \leq k^{\prime} \leq r^{\prime}-1,2 \leq r^{\prime} \leq n-1 ; \\
& \left.\phi_{j 0}\left(g_{1}, g_{2}\right), 1 \leq j \leq n-1 ; \phi_{j j}\left(g_{1}, g_{2}\right), 1 \leq j \leq n-1\right) .
\end{aligned}
$$

Remark 4.19. Consider the map $\widetilde{\Phi}: I\left(g_{1}^{\circ}, g_{2}^{\circ}\right) \ni\left(g_{1}, g_{2}\right) \mapsto\left(\phi_{r^{\prime} k^{\prime}}\left(g_{1}, g_{2}\right), 1 \leq\right.$ $k^{\prime} \leq r^{\prime}-1,2 \leq r^{\prime} \leq n-1 ; \phi_{j 0}\left(g_{1}, g_{2}\right), 1 \leq j \leq n-1 ; \phi_{j j}\left(g_{1}, g_{2}\right), 1 \leq j \leq$ $n-1) \in \mathbf{C}^{g_{c}+2(n-1)} / \Lambda$ which is part of the linearization map defined above, and let $p: \mathbf{C}^{g_{c}+2(n-1)} / \Lambda \rightarrow \mathbf{C}^{g_{c}+n-1} / \Lambda^{\prime}$ be the natural projection onto the first $g_{c}+n-1$ components (here $\Lambda^{\prime}$ is the lattice in $\mathbf{C}^{g_{c}+n-1}$ generated by the column vectors of 
the submatrix $\left(\begin{array}{cc}L_{1} & 0 \\ L_{2} & L_{3}\end{array}\right)$ of $\left.L\right)$. We can interpret the non-compact abelian Lie group $\mathbf{C}^{g_{c}+n-1} / \Lambda^{\prime}$ and the map $p \circ \widetilde{\Phi}$ in terms of known constructs in algebraic geometry as follows. Let $S$ be the support of the divisor $P_{+}$. By identifying the $n$ points of $S$ as one, we obtain the singular curve $\mathcal{C}_{P_{+}}\left(g_{1}^{\circ}, g_{2}^{\circ}\right)=\left(\mathcal{C}\left(g_{1}^{\circ}, g_{2}^{\circ}\right) \backslash S\right) \cup\{Q\}$, where $Q$ is its unique singular point. From the theory developed in [R1], [R2] (see also [S]), the dimension of the space $\Omega\left(-P_{+}\right)$of everywhere regular differentials on $\mathcal{C}_{P_{+}}\left(g_{1}^{\circ}, g_{2}^{\circ}\right)$ is given by its arithmetic genus $p_{a}\left(\mathcal{C}_{P_{+}}\left(g_{1}^{\circ}, g_{2}^{\circ}\right)\right)=\pi=g_{c}+n-1$. Moreover, a 1 -form $\omega$ on the curve $\mathcal{C}\left(g_{1}^{\circ}, g_{2}^{\circ}\right)$ is regular at $Q$ iff $\sum_{P \in S} \operatorname{res}_{P} \omega=0$ and $\operatorname{ord}_{P} \omega \geq-1$ for all $P \in S$. By this criterion, the 1 -forms $\omega_{2,-1}, \ldots, \omega_{n,-1}$ are everywhere regular differentials on $\mathcal{C}_{P_{+}}\left(g_{1}^{\circ}, g_{2}^{\circ}\right)$, the set $\left\{\omega_{r k} \mid k+3 \leq r \leq n, k=-1, \ldots, n-3\right\}$ is a basis of $\Omega\left(-P_{+}\right)$, the abelian complex Lie group $\mathbf{C}^{g_{c}+n-1} / \Lambda^{\prime}$ is the generalized Jacobian $J_{P_{+}}$in the notation of [S], and the map $\mathbf{A}_{P_{+}}: \mathcal{C}\left(g_{1}^{\circ}, g_{2}^{\circ}\right) \backslash S \rightarrow J_{P_{+}}, P \mapsto$ $\left(\int_{P_{0}}^{P} \omega_{r k}, k+3 \leq r \leq n, k=0, \ldots, n-3 ; \int_{P_{0}}^{P} \omega_{j+1,-1}, j=1, \ldots, n-1\right)$ is the generalized Abel-Jacobi map, for which there exists an analogue of the classical Jacobi inversion theorem [R2]. As in the classical case, $\mathbf{A}_{P_{+}}$can be extended to a map from the group of divisors prime to $S$ to $J_{P_{+}}$, and we shall denote this extension again by the same symbol. Now, for $\left(g_{1}, g_{2}\right) \in I\left(g_{1}^{\circ}, g_{2}^{\circ}\right)$, the divisor $D\left(g_{1}, g_{2}\right)$ is prime to $S$ by the invertibility of $g_{1}$ and (GA1). Hence the map $p \circ \widetilde{\Phi}$ is the composite of the map $I\left(g_{1}^{\circ}, g_{2}^{\circ}\right) \ni\left(g_{1}, g_{2}\right) \mapsto D\left(g_{1}, g_{2}\right)$ and the generalized Abel-Jacobi map $\mathbf{A}_{P_{+}}$. On the other hand, the definition of the remaining variables $\phi_{j j}\left(g_{1}, g_{2}\right)$ of the map $\widetilde{\Phi}$ involves the divisor $D^{T}\left(g_{1}, g_{2}\right)$ in addition to $D\left(g_{1}, g_{2}\right)$. For this reason, it is not clear if there is a way to fit these into the above picture.

\section{INJECTIVITY OF THE LINEARIZATION MAP}

Instead of considering the map $\mathbf{L}$ defined at the end of the last section, we shall replace it by a map $\mathbf{L}^{\circ}$ into real variables. Let $I^{c}\left(g_{1}^{\circ}, g_{2}^{\circ}\right)$ denote the connected component of $I\left(g_{1}^{\circ}, g_{2}^{\circ}\right)$ containing $\left(g_{1}^{\circ}, g_{2}^{\circ}\right)$. For each $1 \leq k \leq n-1$, there are two possibilities for the reality of $\lambda_{r k}$, either

$(a)_{k} \quad d_{k}>0$ of the generalized eigenvalues $\lambda_{r k}$ are real, and we order them as follows: $\lambda_{1 k}, \ldots, \lambda_{d_{k} k}, \lambda_{d_{k}+1, k}, \lambda_{d_{k}+2, k}=\bar{\lambda}_{d_{k}+1, k}, \ldots, \lambda_{d_{k}+2 c_{k}-1, k}, \lambda_{d_{k}+2 c_{k}, k}=$ $\bar{\lambda}_{d_{k}+2 c_{k}-1, k}$

or

$(b)_{k}$ all the generalized eigenvalues $\lambda_{r k}$ are complex, i.e. $d_{k}=0$, and we order them as $\lambda_{1 k}, \lambda_{2 k}=\bar{\lambda}_{1 k}, \lambda_{3 k}, \lambda_{4 k}=\bar{\lambda}_{3 k}, \ldots$

For each $1 \leq k \leq n-1$, define $\Theta_{k}: I^{c}\left(g_{1}^{\circ}, g_{2}^{\circ}\right) \rightarrow \mathbf{R}^{d_{k}-1} \times \mathbf{R}^{c_{k}} \times \mathbf{T}^{c_{k}}$ by

$$
\begin{array}{r}
\Theta_{k}\left(g_{1}, g_{2}\right)=\left(\frac{1}{\lambda_{2 k}} \log \left|\frac{m_{2 k}\left(g_{1}, g_{2}\right)}{m_{1 k}\left(g_{1}, g_{2}\right)}\right|, \ldots, \frac{1}{\lambda_{d_{k}, k}} \log \left|\frac{m_{d_{k, k}}\left(g_{1}, g_{2}\right)}{m_{1 k}\left(g_{1}, g_{2}\right)}\right|\right. \\
\frac{1}{\left|\lambda_{d_{k}+1, k}\right|} \log \left|\frac{m_{d_{k}+1, k}\left(g_{1}, g_{2}\right)}{m_{1 k}\left(g_{1}, g_{2}\right)}\right|, \frac{1}{\left|\lambda_{d_{k}+3, k}\right|} \log \left|\frac{m_{d_{k}+3, k}\left(g_{1}, g_{2}\right)}{m_{1 k}\left(g_{1}, g_{2}\right)}\right| \\
\left.\ldots ; \arg m_{d_{k}+1, k}\left(g_{1}, g_{2}\right), \arg m_{d_{k}+3, k}\left(g_{1}, g_{2}\right), \ldots\right)
\end{array}
$$


in case $(a)_{k}$ and $\Theta_{k}: I^{c}\left(g_{1}^{\circ}, g_{2}^{\circ}\right) \rightarrow \mathbf{R}^{c_{k}-1} \times \mathbf{T}^{c_{k}}$ by

$$
\begin{array}{r}
\Theta_{k}\left(g_{1}, g_{2}\right)=\left(\frac{1}{\left|\lambda_{3 k}\right|} \log \left|\frac{m_{3 k}\left(g_{1}, g_{2}\right)}{m_{1 k}\left(g_{1}, g_{2}\right)}\right|, \ldots, \frac{1}{\left|\lambda_{2 c_{k}-1, k}\right|} \log \left|\frac{m_{2 c_{k}-1, k}\left(g_{1}, g_{2}\right)}{m_{1 k}\left(g_{1}, g_{2}\right)}\right| ;\right. \\
\left.\arg m_{1 k}\left(g_{1}, g_{2}\right), \arg m_{3 k}\left(g_{1}, g_{2}\right), \ldots, \arg m_{2 c_{k}-1, k}\left(g_{1}, g_{2}\right)\right)
\end{array}
$$

in case $(b)_{k}$

Putting the $\Theta_{k}$ 's together, we have a map

$$
\begin{gathered}
\Theta: I^{c}\left(g_{1}^{\circ}, g_{2}^{\circ}\right) \rightarrow \mathbf{R}^{\frac{1}{2}(n-1)(n-2)-\sum_{i} c_{i}} \times \mathbf{T}^{\sum_{k} c_{k}}, \\
\Theta\left(g_{1}, g_{2}\right)=\left(\Theta_{1}\left(g_{1}, g_{2}\right), \ldots, \Theta_{n-1}\left(g_{1}, g_{2}\right)\right) .
\end{gathered}
$$

Now, replace $\phi_{r k}\left(g_{1}, g_{2}\right)$ by $\phi_{r k}^{\circ}\left(g_{1}, g_{2}\right)=\int_{D\left(g_{1}^{\circ}, g_{2}^{\circ}\right)}^{D\left(g_{1}, g_{2}\right)} \omega_{r+1, k-1}, k \geq 0$. Then $\phi_{r k}^{\circ}$ and $\phi_{r r}$ are real-valued $(\bmod \Lambda)$. Therefore, we can define

$$
\begin{gathered}
\Phi: I^{c}\left(g_{1}^{\circ}, g_{2}^{\circ}\right) \rightarrow \mathbf{R}^{\frac{1}{2}(n-1)(n-2)+2(n-1)} / \Lambda, \\
\Phi\left(g_{1}^{\circ}, g_{2}^{\circ}\right)=\left(\phi_{r k}^{\circ}\left(g_{1}, g_{2}\right), 1 \leq k \leq r-1,2 \leq r \leq n-1 ;\right. \\
\left.\phi_{j 0}^{\circ}\left(g_{1}, g_{2}\right), 1 \leq j \leq n-1 ; \phi_{j j}\left(g_{1}, g_{2}\right), 1 \leq j \leq n-1\right) .
\end{gathered}
$$

Putting $\Theta$ and $\Phi$ together, we obtain the map

$$
\mathbf{L}^{\circ}: I^{c}\left(g_{1}^{\circ}, g_{2}^{\circ}\right) \rightarrow \mathbf{R}^{\frac{1}{2}(n-1)(n-2)-\sum_{i} c_{i}} \times \mathbf{T}^{\sum c_{i}} \times\left(\mathbf{R}^{\frac{1}{2}(n-1)(n-2)+2(n-1)} / \Lambda\right)
$$

i.e.

$$
\mathbf{L}^{\circ}\left(g_{1}, g_{2}\right)=\left(\Theta\left(g_{1}, g_{2}\right), \Phi\left(g_{1}, g_{2}\right)\right) .
$$

The goal of this section is to establish the injectivity of $\mathbf{L}^{\circ}$.

For $\left(g_{1}, g_{2}\right) \in I^{c}\left(g_{1}^{\circ}, g_{2}^{\circ}\right)$, consider the generalized Schur decomposition [MS]

$$
g_{1}=u_{1} \ell_{1} u_{2}^{*}, g_{2}=u_{2} \ell_{2} u_{1}^{*},
$$

where $u_{1}, u_{2}$ are unitary, $\ell_{1}, \ell_{2}$ are lower triangular. The diagonal entries of $\ell_{2} \ell_{1}$ are the eigenvalues of $g_{2} g_{1}$ in some order which will be fixed in the subsequent discussion. As $\ell_{2} \ell_{2}^{*}$ is positive definite,

$$
\ell_{2} \ell_{2}^{*}=O \mu O^{*},
$$

where $O$ is unitary and $\mu=\operatorname{diag}\left(\mu_{1}, \ldots, \mu_{n}\right)$. The entries of $\mu$ in some order will also be fixed in what follows; they are clearly the eigenvalues of $g_{2} g_{2}^{T}$. We shall choose $O$ in such a way that $u_{2} O$ is a real matrix. This is possible as $g_{2} g_{2}^{T}$ is a real matrix.

We now extend the definitions in section 3 to complex matrices $w_{1}, w_{2} \in G L(n, \mathbf{C})$ in the obvious way, i.e.

$$
\begin{aligned}
J\left(w_{1}, w_{2} ; h, z\right) & =\operatorname{det}\left(w_{2} w_{1}-h w_{2} w_{2}^{*}-z\right), \\
M_{h, z}\left(w_{1} w_{2}\right) & =w_{2} w_{1}-h w_{2} w_{2}^{*}-z .
\end{aligned}
$$

Then, with the notation in (5.5) and (5.6), we have

$$
\begin{aligned}
J\left(g_{1}, g_{2} ; h, z\right) & =J\left(\ell_{1}, \ell_{2} ; h, z\right)=J\left(O^{*} \ell_{1} O, O^{*} \ell_{2} O ; h, z\right), \\
M_{h, z}\left(g_{1}, g_{2}\right) & =u_{2} M_{h, z}\left(\ell_{1}, \ell_{2}\right) u_{2}^{*}=u_{2} O M_{h, z}\left(O^{*} \ell_{1} O, O^{*} \ell_{2} O\right)\left(u_{2} O\right)^{T} .
\end{aligned}
$$


Consequently, the kernel vectors $f\left(g_{1}, g_{2} ; p\right)$ and $f\left(O^{*} \ell_{1} O, O^{*} \ell_{2} O ; p\right)$ are related by

$$
f\left(g_{1}, g_{2} ; p\right)=u_{2} O f\left(O^{*} \ell_{1} O, O^{*} \ell_{2} O ; p\right) .
$$

Clearly, the divisor $D\left(O^{*} \ell_{1} O, O^{*} \ell_{2} O\right)$ contains the points

$$
P_{-}^{i}=\left[0:-1: \mu_{i}\right], \quad i=2, \ldots, n,
$$

and so

$$
D\left(O^{*} \ell_{1} O, O^{*} \ell_{2} O\right)=D^{\prime}\left(O^{*} \ell_{1} O, O^{*} \ell_{2} O\right)+\sum_{i=2}^{n} P_{-}^{i} .
$$

The following lemma is a special case of a general result which has been discussed by a number of authors [RSTS], [V] in various languages.

Lemma 5.12. The divisor $D^{\prime}\left(O^{*} \ell_{1} O, O^{*} \ell_{2} O\right)$ is regular.

Corollary 5.13. If $\widehat{f}_{j}(p)=\left(e_{j}, f\left(O^{*} \ell_{1} O, O^{*} \ell_{2} O ; p\right)\right) /\left(e_{1}, f\left(O^{*} \ell_{1} O, O^{*} \ell_{2} O ; p\right)\right)$, $j \geq 2$, then

$$
\left(\widehat{f}_{j}\right) \geq-D^{\prime}\left(O^{*} \ell_{1} O, O^{*} \ell_{2} O\right)+P_{-}^{1}-P_{-}^{j}
$$

and the divisor $D^{\prime}\left(O^{*} \ell_{1} O, O^{*} \ell_{2} O\right)-P_{-}^{1}+P_{-}^{j}$ is regular.

To show that the map $\mathbf{L}^{\circ}$ is injective, suppose $\left(g_{1}, g_{2}\right),\left(\tilde{g}_{1}, \tilde{g}_{2}\right) \in I^{c}\left(g_{1}^{\circ}, g_{2}^{\circ}\right)$ and $\mathbf{L}^{\circ}\left(g_{1}, g_{2}\right)=\mathbf{L}^{\circ}\left(\tilde{g}_{1}, \tilde{g}_{2}\right)$. Consider the generalized Schur decompositions

$$
\begin{aligned}
& g_{1}=u_{1} \ell_{1} u_{2}^{*}, \quad g_{2}=u_{2} \ell_{2} u_{1}^{*}, \\
& \tilde{g}_{1}=\tilde{u}_{1} \tilde{\ell}_{1} \tilde{u}_{2}^{*}, \quad g_{2}=\tilde{u}_{2} \tilde{\ell}_{2} \tilde{u}_{1}^{*},
\end{aligned}
$$

where $\ell_{2} \ell_{1}, \tilde{\ell}_{2} \tilde{\ell}_{1}$ have the same diagonal part. Corresponding to $\ell_{2}$ and $\tilde{\ell}_{2}$, there exist unitary matrices $O$ and $\tilde{O}$ such that

$$
\ell_{2} \ell_{2}^{*}=O \mu O^{*}, \quad \tilde{\ell_{2}} \tilde{\ell}_{2}^{*}=\tilde{O} \mu \tilde{O}^{*}, \quad \mu=\operatorname{diag}\left(\mu_{1}, \ldots, \mu_{n}\right) .
$$

Moreover, $O$ and $\tilde{O}$ are chosen so that the matrices $u_{2} O$ and $\tilde{u}_{2} \tilde{O}$ are real.

Step 1. $D\left(O^{*} \ell_{1} O, O^{*} \ell_{2} O\right)=D\left(\tilde{O}^{*} \tilde{\ell_{1}} \tilde{O}, \tilde{O}^{*} \tilde{\ell_{2}} \tilde{O}\right)$.

Proof. From $\phi_{r k}^{\circ}\left(g_{1}, g_{2}\right)=\phi_{r k}^{\circ}\left(\tilde{g}_{1}, \tilde{g}_{2}\right), k \geq 1$, and Abel's theorem, we have

$$
0=\int_{D\left(g_{1}, g_{2}\right)}^{D\left(\tilde{g}_{1}, \tilde{g}_{2}\right)} \omega_{r+1, k-1}=\int_{D^{\prime}\left(O^{*} \ell_{1} O, O^{*} \ell_{2} O\right)}^{D^{\prime}\left(\tilde{O}^{*} \tilde{\ell_{1}} \tilde{O}, \tilde{O}^{*} \tilde{\ell_{2}} \tilde{O}\right)} \omega_{r+1, k-1} .
$$

Since $\left\{\omega_{r+1, k-1} \mid 1 \leq k \leq r-1,2 \leq r \leq n-1\right\}$ is a basis of $H^{\circ}\left(\mathcal{C}\left(g_{1}^{\circ}, g_{2}^{\circ}\right), \Omega\right)$, it follows from the converse of Abel's theorem that there exists $\phi$ meromorphic on $\mathcal{C}\left(g_{1}^{\circ}, g_{2}^{\circ}\right)$ such that $(\phi)=-D^{\prime}\left(O^{*} \ell_{1} O, O^{*} \ell_{2} O\right)+D^{\prime}\left(\tilde{O}^{*} \tilde{\ell_{1}} \tilde{O}, \tilde{O}^{*} \tilde{\ell_{2}} \tilde{O}\right)$. But then $\phi$ must be constant by Lemma 5.12, and so the assertion follows.

Step 2. $\hat{f}_{j}\left(\tilde{O}^{*} \tilde{\ell_{1}} \tilde{O}, \tilde{O}^{*} \tilde{\ell_{2}} \tilde{O} ; p\right)=\alpha_{j} \hat{f}_{j}\left(O^{*} \ell_{1} O, O^{*} \ell_{2} O ; p\right), \alpha_{j} \neq 0$.

Proof. This is immediate from Step 1 and Corollary 5.13.

In particular, $\alpha_{1}=1$. Let $\alpha=\operatorname{diag}\left(\alpha_{1}, \ldots, \alpha_{n}\right)$.

Step 3. $D\left(g_{1}, g_{2}\right)=D\left(\tilde{g}_{1}, \tilde{g}_{2}\right), D^{T}\left(g_{1}, g_{2}\right)=D^{T}\left(\tilde{g}_{1}, \tilde{g}_{2}\right)$. 
Proof. Let $f(p)=f\left(O^{*} \ell_{1} O, O^{*} \ell_{2} O ; p\right)$ and $f^{T}(p)=f^{T}\left(O^{*} \ell_{1} O, O^{*} \ell_{2} O ; p\right)$. As in (5.9), we have $f^{T}\left(g_{1}, g_{2} ; p\right)=u_{2} O f^{T}\left(O^{*} \ell_{1} O, O^{*} \ell_{2} O ; p\right)$. Therefore, the divisor of the meromorphic function $v(p)=\left(e_{1}, u_{2} O f(p)\right) /\left(e_{1}, \tilde{u}_{2} \tilde{O} \alpha f(p)\right)$ is given by

$$
(v)=D\left(g_{1}, g_{2}\right)-D\left(\tilde{g}_{1}, \tilde{g}_{2}\right),
$$

where we have used the result in Step 2. Similarly, if we define

$$
w(p)=\frac{\left(e_{1}, u_{2} O f(p)\right)}{\left(e_{1}, u_{2} O f^{T}(p)\right)} \cdot \frac{\left(e_{1}, \tilde{u}_{2} \tilde{O} \alpha^{-1} f^{T}(p)\right)}{\left(e_{1}, \tilde{u}_{2} \tilde{O} \alpha f^{T}(p)\right)},
$$

then

$$
(w)=D\left(g_{1}, g_{2}\right)+D^{T}\left(g_{1}, g_{2}\right)-D\left(\tilde{g}_{1}, \tilde{g}_{2}\right)-D^{T}\left(g_{1}, g_{2}\right) .
$$

By the definition of the trace $[F]$, we can choose paths of integration $\gamma_{1}$ and $\gamma_{2}$ in $\mathbf{C P}^{1}$ from 0 to $\infty$ (independent of $j$ and $r$ respectively) such that

$$
\begin{aligned}
\int_{D\left(g_{1}, g_{2}\right)}^{D\left(\tilde{g}_{1}, \tilde{g}_{2}\right)} \omega_{j+1,-1} & \equiv \int_{v^{-1}\left(\gamma_{1}\right)} \omega_{j+1,-1}=\int_{\gamma_{1}} \operatorname{Trace}\left(\omega_{j+1,-1}\right) \\
& =\int_{0}^{\infty} \sum_{i=1}^{n} \frac{a_{i j}}{\lambda-v\left(P_{+}^{i}\right)} d \lambda, a_{i j}=\operatorname{res}_{P_{+}^{i}} \omega_{j+1,-1} \\
& =\sum_{i=1}^{n} a_{i j} \log \left(-v\left(P_{+}^{i}\right)\right)
\end{aligned}
$$

and

$$
\begin{aligned}
\int_{D^{T}\left(\tilde{g}_{1}, \tilde{g}_{2}\right)+D\left(g_{1}, g_{2}\right)}^{D\left(\tilde{g}_{1}, \tilde{g}_{2}\right)+D^{T}\left(g_{1}, g_{2}\right)} \omega_{r+1, r-1} & \equiv \int_{w^{-1}\left(\gamma_{2}\right)} \omega_{r+1, r-1}=\int_{\gamma_{2}} \operatorname{Trace}\left(\omega_{r+1, r-1}\right) \\
& =\int_{0}^{\infty} \sum_{i=1}^{n} \frac{b_{i r}}{\lambda-w\left(P_{-}^{i}\right)}, \quad b_{i r}=\operatorname{res}_{P_{-}^{i}} \omega_{r+1, r-1} \\
& =\sum_{i=1}^{n} b_{i r} \log \left(-w\left(P_{-}^{i}\right)\right) .
\end{aligned}
$$

Equivalently,

$$
\begin{array}{r}
\int_{D\left(g_{1}, g_{2}\right)}^{D\left(\tilde{g}_{1}, \tilde{g}_{2}\right)}\left(\omega_{2,-1}, \ldots, \omega_{n,-1}\right)^{T}=L_{3} \phi, \\
\int_{D^{T}\left(\tilde{g}_{1}, \tilde{g}_{2}\right)+D\left(g_{1}, g_{2}\right)}^{D\left(\tilde{g}_{1}, \tilde{g}_{2}\right)+D^{T}\left(g_{1}, g_{2}\right)}\left(\omega_{2,0}, \ldots, \omega_{n, n-2}\right)^{T}=L_{5} \psi,
\end{array}
$$

where

$$
\phi=\frac{1}{2 \pi \sqrt{-1}}\left(\log \left(-v\left(P_{+}^{1}\right)\right), \ldots, \log \left(-v\left(P_{+}^{n}\right)\right)\right)^{T}
$$

and

$$
\psi=\frac{1}{2 \pi \sqrt{-1}}\left(\log \left(-w\left(P_{-}^{1}\right)\right), \ldots, \log \left(-w\left(P_{-}^{n}\right)\right)\right)^{T} .
$$


On the other hand, from $\Phi\left(g_{1}, g_{2}\right)=\Phi\left(\tilde{g}_{1}, \tilde{g}_{2}\right)$, we have

$$
\begin{aligned}
\left(\overrightarrow{0}, \int_{D\left(g_{1}, g_{2}\right)}^{D\left(\tilde{g}_{1}, \tilde{g}_{2}\right)}\right. & \left.\omega_{2,-1}, \ldots, \int_{D^{T}\left(\tilde{g}_{1}, \tilde{g}_{2}\right)+D\left(g_{1}, g_{2}\right)}^{D\left(\tilde{g}_{1}, \tilde{g}_{2}\right)+D^{T}\left(g_{1}, g_{2}\right)} \omega_{2,0}, \ldots\right)^{T} \\
& =\left(\begin{array}{ccc}
L_{1} & 0 & 0 \\
L_{2} & L_{3} & 0 \\
L_{4} & 0 & L_{5}
\end{array}\right)\left(\begin{array}{c}
\ell \\
\ell^{\prime} \\
\ell^{\prime \prime}
\end{array}\right)
\end{aligned}
$$

where $\overrightarrow{0} \in \mathbf{R}^{g_{c}}, \ell \in \mathbf{Z}^{2 g_{c}}, \ell^{\prime}, \ell^{\prime \prime} \in \mathbf{Z}^{n}$. As the columns of $L_{1}$ span a lattice in $\mathbf{C}^{g_{c}}$, $\ell$ must be zero and hence $L_{3} \phi=L_{3} \ell^{\prime}, L_{5} \psi=L_{5} \ell^{\prime \prime}$. Consequently, $\phi-\ell^{\prime}=c \mathbf{1}$ and $\psi-\ell^{\prime \prime}=\tilde{c} \mathbf{1}$ for some $c, \tilde{c} \in \mathbf{C}$. Thus, $v\left(P_{+}^{i}\right)=-e^{2 \pi \sqrt{-1} c}, w\left(P_{-}^{i}\right)=-e^{2 \pi \sqrt{-1} \tilde{c}}$, independent of $i$. As a result, we have

$$
\left(u_{2} O\right)^{T} e_{1}+\left(\tilde{u}_{2} \tilde{O} \alpha\right)^{*} e^{2 \pi \sqrt{-1} c} e_{1}=0, \quad\left(\tilde{u}_{2} \tilde{O} \alpha^{-1}\right)^{*} e_{1}+e^{2 \pi \sqrt{-1} \tilde{c}}\left(\tilde{u}_{2} \tilde{O} \alpha\right)^{*} e_{1}=0
$$

which in turn implies that $v(p)=-e^{2 \pi \sqrt{-1} c}, w(p)=-e^{2 \pi \sqrt{-1} c}$. Hence we have

$$
D\left(g_{1}, g_{2}\right)=D\left(\tilde{g}_{1}, \tilde{g}_{2}\right), \quad D^{T}\left(g_{1}, g_{2}\right)=D^{T}\left(\tilde{g}_{1}, \tilde{g}_{2}\right) .
$$

Step 4. $\alpha^{2}=1$.

Proof. From Step 3, $w\left(P_{-}^{i}\right)=-e^{2 \pi \sqrt{-1} \tilde{c}}$ independent of $i$. Now $w\left(P_{-}^{i}\right)=1 / \alpha_{i}^{2}$ and as $\alpha_{1}=1$, it follows that $\alpha_{i}^{2}=1$ for all $i$.

Step 5. $\tilde{\ell}_{1}=\zeta \ell_{1} \eta^{*}, \tilde{\ell}_{2}=\eta \ell_{2} \zeta^{*}$, where $\zeta$ and $\eta$ are unitary diagonal.

Proof. From Step 2 and Step 4, we obtain $O^{*} \tilde{\ell}_{2} \tilde{\ell}_{1} \tilde{O}=\alpha O^{*} \ell_{2} \ell_{1} O \alpha^{-1}$, where $\alpha^{2}=1$. Equivalently, $\tilde{\ell}_{2} \tilde{\ell}_{1} \tilde{O} \alpha O^{*}=\tilde{O} \alpha O^{*} \ell_{2} \ell_{1}$. The upshot of this is that $\tilde{O} \alpha O^{*}$ must be lower triangular as the matrices $\ell_{2} \ell_{1}, \tilde{\ell}_{2} \tilde{\ell}_{1}$ are invertible and have distinct eigenvalues. Hence, $\tilde{O} \alpha O^{*}=\eta$, where $\eta$ is unitary diagonal. But then we have $\tilde{\ell}_{2} \tilde{\ell}_{1}=\eta \ell_{2} \ell_{1} \eta^{*}, \tilde{\ell}_{2} \tilde{\ell}_{2}^{*}=\tilde{O} \mu \tilde{O}^{*}=\eta O \alpha^{-1} \mu \alpha O^{*} \eta^{*}=\eta O \mu O^{*} \eta=\left(\eta \ell_{2}\right)\left(\eta \ell_{2}\right)^{*}$. Consequently, $\tilde{\ell}_{2}=\eta \ell_{2} \zeta^{*}$, where $\zeta$ is unitary diagonal, and this implies $\tilde{\ell}_{1}=\zeta \ell_{1} \eta^{*}$.

Summarizing the above results, we have

Proposition 5.14. If $\mathbf{L}^{\circ}\left(g_{1}, g_{2}\right)=\mathbf{L}^{\circ}\left(\tilde{g}_{1}, \tilde{g}_{2}\right)$, then $\left(g_{1}, g_{2}\right)$ and $\left(\tilde{g}_{1}, \tilde{g}_{2}\right)$ have generalized Schur decompositions $g_{1}=u_{1} \ell_{1} u_{2}^{*}, g_{2}=u_{2} \ell_{2} u_{1}^{*}$ and $\tilde{g}_{1}=\tilde{u}_{1} \ell_{1} \tilde{u}_{2}^{*}, \tilde{g}_{2}=$ $\tilde{u}_{2} \ell_{2} \tilde{u}_{1}^{*}$ respectively with the same lower triangular factors $\ell_{1}, \ell_{2}$.

Step 6. The first rows of $u_{2}$, and $\tilde{u}_{2}$ differ by a multiplicative phase factor, and therefore we can assume these first rows are equal.

Proof. Let $f(p)$ now denote $f\left(\ell_{1}, \ell_{2} ; p\right)$ and let $s(p)=\left(e_{1}, \tilde{u}_{2} f(p)\right) /\left(e_{1}, u_{2} f(p)\right)$. Then $(s)=D\left(g_{1}, g_{2}\right)-D\left(\tilde{g}_{1}, \tilde{g}_{2}\right)=0$ by Step 3, which implies $s$ is constant. As a result, $\tilde{u}_{2}^{*} e_{1}+e^{i \alpha} u_{2}^{*} e_{1}=0$ for some $\alpha \in \mathbf{R}$.

Step 7. $\tilde{u}_{1}=\rho_{1} u_{1}, \tilde{u}_{2}=\rho_{2} u_{2}$, where $\rho_{1}, \rho_{2}$ are unitary diagonal.

Proof. This step makes use of the equality of $\lambda_{r k}$ and $\theta_{r k}$ of the elements $\left(g_{1}, g_{2}\right)$, $\left(\tilde{g}_{1}, \tilde{g}_{2}\right)$, and proceeds as in the proof of Theorem 5.1 in [L3]. The row vectors of $u_{1}$ (resp. $\tilde{u}_{1}$ ) and $u_{2}$ (resp. $\tilde{u}_{2}$ ) are obtained one at a time by solving linear equations with a quadratic constraint. The uniqueness of these row vectors up to a phase factor follows by invoking local uniqueness (Corollary 4.17).

Thus it follows from Step 7 that $\tilde{g}_{1}=\rho_{1} g_{1} \bar{\rho}_{2}, \quad \tilde{g}_{2}=\rho_{2} g_{2} \bar{\rho}_{1}$, where $\rho_{1}, \rho_{2}$ are unitary diagonal. 
Step 8. $\tilde{g}_{1}=\sigma_{1} g_{1} \sigma_{2}, \tilde{g}_{2}=\sigma_{2} g_{2} \sigma_{1}, \sigma_{1}, \sigma_{2}$ are diagonal matrices with \pm 1 on the diagonal, $\left(\sigma_{1}\right)_{n n}=1$, and $\sigma_{2}=w^{*} \sigma_{1} w^{*}$, where $w^{*}=\left(\delta_{i, n+1-j}\right)$.

Proof. By the reality of the matrices $g_{1}, \tilde{g}_{1}, g_{2}$ and $\tilde{g}_{2}$, we have $g_{1}=\bar{\rho}_{1}^{2} g_{1} \rho_{2}^{2}$ and $g_{2}=\bar{\rho}_{2}^{2} g_{2} \rho_{1}^{2}$. Therefore, if $\left(g_{1}\right)_{i j} \neq 0$, then $\left(\rho_{1}^{2}\right)_{i i}=\left(\rho_{2}^{2}\right)_{j j}$. Consider the adjacency graph associated to $g_{1}$. If this has a single component, then $\rho_{1}^{2}=\rho_{2}^{2}=\beta^{2} I$ for some phase $\beta$. In this case, $\rho_{1}=\beta \sigma_{1}, \rho_{2}=\beta \sigma_{2}$, where $\sigma_{1}, \sigma_{2}$ are diagonal matrices with \pm 1 on the diagonal. The same argument can be applied to each component of the adjacency graph of $g_{1}$, yielding $\tilde{g}_{1}=\sigma_{1} g_{1} \sigma_{2}$ and $\tilde{g}_{2}=\sigma_{2} g_{2} \sigma_{1}$ in the general case. Clearly we can take $\left(\sigma_{1}\right)_{n, n}=1$. Finally, from $\operatorname{sgn} \operatorname{det}\left(\tilde{g}_{2}\right)_{k}=\operatorname{sgn} \operatorname{det}\left(g_{2}\right)_{k}, k=$ $0,1, \ldots, n-1$, we infer that $\sigma_{2}=w^{*} \sigma_{1} w^{*}$.

Step 9. $\tilde{g}_{1}=g_{1}, \tilde{g}_{2}=g_{2}$.

Proof. From Step 8, $\left(\sigma_{1}\right)_{n, n}=\left(\sigma_{2}\right)_{1,1}=1$. To show that $\sigma_{1}=\sigma_{2}=I$, we will need the formula [L3]

$$
\begin{aligned}
& m_{r k}\left(g_{1}^{\prime}, g_{2}^{\prime}\right) \\
& \quad=(-1)^{k-1} \frac{\left.\left(e_{1} \wedge \cdots \wedge e_{k-1} \wedge e_{k+1},\left(\left(g_{1}^{\prime}-\lambda_{r k} g_{2}^{\prime}\right)^{-1}\right)^{-1}\right)^{\wedge k} e_{n-k+1} \wedge \cdots \wedge e_{n}\right)}{\left(e_{1} \wedge \cdots \wedge e_{k},\left(\left(g_{1}^{\prime}-\lambda_{r k} g_{2}^{\prime-1}\right)^{-1}\right)^{\wedge(k-1)} e_{n-k+2} \wedge \cdots \wedge e_{n}\right)},
\end{aligned}
$$

$k \geq 2$. First, suppose $(a)_{1}$ holds, i.e. $d_{1}>0$. Then

$$
m_{11}\left(g_{1}^{\prime}, g_{2}^{\prime}\right)=\left(e_{2},\left(g_{1}^{\prime}-\lambda_{1}, g_{2}^{\prime-1}\right)^{-1} e_{n}\right)
$$

is real and has constant sign for all $\left(g_{1}^{\prime}, g_{2}^{\prime}\right) \in I^{c}\left(g_{1}^{\circ}, g_{2}^{\circ}\right)$. As

$$
m_{11}\left(\tilde{g}_{1}, \tilde{g}_{2}\right)=\left(\sigma_{2}\right)_{2,2}\left(\sigma_{1}\right)_{n, n} m_{11}\left(g_{1}, g_{2}\right)
$$

and $\left(\sigma_{1}\right)_{n, n}=1$, it follows that $\left(\sigma_{2}\right)_{2,2}=\left(\sigma_{1}\right)_{n-1, n-1}=1$. Now suppose that $(b)_{1}$ holds. In this case, the fact that $\arg m_{11}\left(\tilde{g}_{1}, \tilde{g}_{2}\right)=\arg m_{11}\left(g_{1}, g_{2}\right)(\bmod 2 \pi)$ implies $\left(\sigma_{2}\right)_{2,2}=\left(\sigma_{1}\right)_{n-1, n-1}=1$. So in both cases, $\left(\sigma_{2}\right)_{2,2}=\left(\sigma_{1}\right)_{n-1, n-1}=1$. Next, consider $k=2$. We have

$$
m_{12}\left(g_{1}^{\prime}, g_{2}^{\prime}\right)=\frac{-\left(e_{1} \wedge e_{3},\left(\left(g_{1}^{\prime}-\lambda_{12} g_{2}^{\prime-1}\right)^{-1}\right)^{\wedge 2} e_{n-1} \wedge e_{n}\right)}{\left(e_{1},\left(g_{1}^{\prime}-\lambda_{12} g_{2}^{\prime-1}\right)^{-1} e_{n}\right)}
$$

for $\left(g_{1}^{\prime}, g_{2}^{\prime}\right) \in I^{c}\left(g_{1}^{\circ}, g_{2}^{\circ}\right)$. If $(a)_{2}$ holds, then $m_{12}\left(g_{1}^{\prime}, g_{2}^{\prime}\right)$ is real and has constant sign in $I^{c}\left(g_{1}^{\circ}, g_{2}^{\circ}\right)$. As

$$
m_{12}\left(\tilde{g}_{1}, \tilde{g}_{2}\right)=\left(\sigma_{2}\right)_{3,3}\left(\sigma_{1}\right)_{n-1, n-1} m_{12}\left(g_{1}, g_{2}\right),
$$

it follows that $\left(\sigma_{2}\right)_{3,3}=\left(\sigma_{1}\right)_{n-2, n-2}=1$. On the other hand, if $(b)_{2}$ holds, then $\arg m_{k}\left(\tilde{g}_{1}, \tilde{g}_{2}\right)=\arg m_{12}\left(g_{1}, g_{2}\right)(\bmod 2 \pi)$ and again we must have $\left(\sigma_{2}\right)_{3,3}=$ $\left(\sigma_{1}\right)_{n-2, n-2}=1$. Proceed inductively, we conclude that $\sigma_{1}=\sigma_{2}=I$, and so $g_{1}=\tilde{g}_{1}, g_{2}=\tilde{g}_{2}$.

\section{Solving the equations Via FaCtorization PRoblems}

In this section, we solve the Hamilton equations generated by the conserved quantities via factorization problems. To be more precise, the $I_{r k}$ flows, the $J_{r 0}$ flows, as well as the $J_{r r}$ flows, can all be solved via factorization problems for the finite dimensional matrix group $G L(n, \mathbf{R})$. On the other hand, for $1 \leq k^{\prime} \leq$ $r^{\prime}-1,1 \leq r^{\prime} \leq n-1$, the $J_{r^{\prime} k^{\prime}}$ flows correspond to linear motions on the Jacobi variety of the associated spectral curve (Proposition 4.9(a)). Consequently, the corresponding equations are solved by means of theta functions. Indeed, the matrix 
pencil $M_{h}\left(g_{1}, g_{2}\right)=g_{2} g_{1}-h g_{2} g_{2}^{T}$ undergoes an isospectral deformation when $g_{1}, g_{2}$ evolve according to a $J_{r^{\prime} k^{\prime}}$ flow, as it should. What is remarkable is the fact that these isospectral deformations are again Hamiltonian and can be solved via factorization problems for the infinite dimensional loop group. Of course, we still have to recover $g_{1}(t), g_{2}(t)$ from the matrix pencil $M_{h}\left(g_{1}(t), g_{2}(t)\right)$, and that will require additional considerations.

We begin by writing down the various equations which we have to solve. To do so, we use (4.1a), (4.1c), (4.2) and (4.3); we find that the Hamilton equations generated by $I_{r k}$ and $J_{r k}$ are given respectively by

$$
\begin{aligned}
& \dot{g}_{1}=\left(\Pi_{\mathfrak{k}}\left(D_{1} I_{r k}\left(g_{1}, g_{2}\right)\right)\right) g_{1}-g_{1}\left(\Pi_{\mathfrak{k}}\left(D_{1}^{\prime} I_{r k}\left(g_{1}, g_{2}\right)\right)\right), \\
& \dot{g}_{2}=\left(\Pi_{\mathfrak{k}}\left(D_{1}^{\prime} I_{r k}\left(g_{1}, g_{2}\right)\right)\right) g_{2}-g_{2}\left(\Pi_{\mathfrak{k}}\left(D_{1} I_{r k}\left(g_{1}, g_{2}\right)\right)\right), k \geq 1,
\end{aligned}
$$

and

$$
\begin{aligned}
\dot{g}_{1}= & \frac{1}{2} g_{1}\left(\Pi_{\mathfrak{r}}\left(D_{1}^{\prime} J_{r k}\left(g_{1}, g_{2}\right)+D_{2} J_{r k}\left(g_{1}, g_{2}\right)\right)\right) \\
& -\frac{1}{2}\left(\Pi_{\mathfrak{r}}\left(D_{1} J_{r k}\left(g_{1}, g_{2}\right)+D_{2}^{\prime} J_{r k}\left(g_{1}, g_{2}\right)\right)\right) g_{1}, \\
\dot{g}_{2}= & \frac{1}{2} g_{2}\left(\Pi_{\mathfrak{r}}\left(D_{2}^{\prime} J_{r k}\left(g_{1}, g_{2}\right)+D_{1} J_{r k}\left(g_{1}, g_{2}\right)\right)\right) \\
& -\frac{1}{2}\left(\Pi_{\mathfrak{l}}\left(D_{2} J_{r k}\left(g_{1}, g_{2}\right)+D_{1}^{\prime} J_{r k}\left(g_{1}, g_{2}\right)\right)\right) g_{2}, k \geq 0 .
\end{aligned}
$$

In the special case of the $J_{r 0}$ flows and $J_{r r}$ flows, the corresponding equations can be simplified somewhat. From the invariance property of $J_{r 0}$, we have $D_{1} J_{r 0}\left(g_{1}, g_{2}\right)=$ $D_{2}^{\prime} J_{r 0}\left(g_{1}, g_{2}\right)$ and $D_{1}^{\prime} J_{r 0}\left(g_{1}, g_{2}\right)=D_{2} J_{r 0}\left(g_{1}, g_{2}\right)$. On the other hand, it is clear from the definition that $J_{r r}$ is independent of $g_{1}$, so that

$$
D_{1} J_{r r}\left(g_{1}, g_{2}\right)=D_{1}^{\prime} J_{r r}\left(g_{1}, g_{2}\right)=0 \text {. }
$$

As a result, it follows from (6.2) that the $J_{r 0}$ flows and $J_{r r}$ flows are defined by

$$
\begin{gathered}
\dot{g}_{1}=\left(\Pi_{\mathfrak{k}}\left(D_{1} J_{r 0}\left(g_{1}, g_{2}\right)\right)\right) g_{1}-g_{1}\left(\Pi_{\mathfrak{k}}\left(D_{2} J_{r 0}\left(g_{1}, g_{2}\right)\right)\right), \\
\dot{g}_{2}=\left(\Pi_{\mathfrak{k}}\left(D_{2} J_{r 0}\left(g_{1}, g_{2}\right)\right)\right) g_{2}-g_{2}\left(\Pi_{\mathfrak{k}}\left(D_{1} J_{r 0}\left(g_{1}, g_{2}\right)\right)\right)
\end{gathered}
$$

and

$$
\begin{aligned}
\dot{g}_{1} & =\frac{1}{2} g_{1}\left(\Pi_{\mathfrak{l}}\left(D_{2} J_{r r}\left(g_{1}, g_{2}\right)\right)\right)-\frac{1}{2}\left(\Pi_{\mathfrak{l}}\left(D_{2}^{\prime} J_{r r}\left(g_{1}, g_{2}\right)\right)\right) g_{1}, \\
\dot{g}_{2} & =\frac{1}{2}\left(\Pi_{\mathfrak{k}}\left(D_{2} J_{r r}\left(g_{1}, g_{2}\right)\right)\right) g_{2}-\frac{1}{2} g_{2}\left(\Pi_{\mathfrak{k}}\left(D_{2}^{\prime} J_{r r}\left(g_{1}, g_{2}\right)\right)\right),
\end{aligned}
$$

respectively.

Theorem 6.5. Let $\left(g_{1}^{\circ}, g_{2}^{\circ}\right) \in \mathcal{U}$. If $Q_{i}(t), L_{i}(t), i=1,2$, are the solutions of the factorization problems

$$
e^{-t D_{1} I_{r k}\left(g_{1}^{\circ}, g_{2}^{\circ}\right)}=Q_{1}(t) L_{1}(t), \quad e^{-t D_{1}^{\prime} I_{r k}\left(g_{1}^{\circ}, g_{2}^{\circ}\right)}=Q_{2}(t) L_{2}(t),
$$

where $Q_{i}(t) \in K, L_{i}(t) \in L$, then the formulas

$$
g_{1}(t)=Q_{1}^{T}(t) g_{1}^{\circ} Q_{2}(t), \quad g_{2}(t)=Q_{2}^{T}(t) g_{2}^{\circ} Q_{1}(t)
$$

give the $I_{r k}$ flow with $g_{1}(0)=g_{1}^{\circ}, g_{2}(0)=g_{2}^{\circ}$. 
Proof. From Proposition 3.18 of [L3], we deduce that

$$
e^{-t D_{1} I_{r k}\left(g_{1}^{\circ}, g_{2}^{\circ}\right)}=\left(\begin{array}{cc}
A & 0 \\
B & I_{k}
\end{array}\right)
$$

while

$$
e^{-t D_{1}^{\prime} I_{r k}\left(g_{1}^{\circ}, g_{2}^{\circ}\right)}=\left(\begin{array}{cc}
I_{k} & 0 \\
C & D
\end{array}\right) .
$$

As $Q_{1}(t), Q_{2}(t)$ are obtained from the exponential matrices above via the GramSchmidt process, it follows that

$$
Q_{1}(t)=\left(\begin{array}{cc}
Q_{11}(t) & 0 \\
0 & I_{k}
\end{array}\right) \quad \text { and } \quad Q_{2}(t)=\left(\begin{array}{cc}
I_{k} & 0 \\
0 & Q_{22}(t)
\end{array}\right) .
$$

Now, by differentiating $g_{1}(t)=Q_{1}^{T}(t) g_{1}^{\circ} Q_{2}(t)$ and $g_{2}(t)=Q_{2}^{T}(t) g_{2}^{\circ} Q_{1}(t)$, we obtain

$$
\dot{g}_{1}=\dot{Q}_{1}^{T} Q_{1} g_{1}-g_{1} \dot{Q}_{2}^{T} Q_{2}, \quad \dot{g}_{2}=\dot{Q}_{2}^{T} Q_{2} g_{2}-g_{2} \dot{Q}_{1}^{T} Q_{1}
$$

To complete the proof, we must show that

$$
\dot{Q}_{1}^{T} Q_{1}=\Pi_{\mathfrak{k}}\left(D_{1} I_{r k}\left(g_{1}, g_{2}\right)\right) \quad \text { and } \quad \dot{Q}_{2}^{T} Q_{2}=\Pi_{\mathfrak{k}}\left(D_{1}^{\prime} I_{r k}\left(g_{1}, g_{2}\right)\right) .
$$

Accordingly, we differentiate (6.6), which yields

$$
\begin{aligned}
& -Q_{1}^{T} \cdot D_{1} I_{r k}\left(g_{1}^{\circ}, g_{2}^{\circ}\right) \cdot Q_{1}=-\dot{Q}_{1}^{T} Q_{1}+\dot{L}_{1} L_{1}^{-1}, \\
& -Q_{2}^{T} \cdot D_{1}^{\prime} I_{r k}\left(g_{1}^{\circ}, g_{2}^{\circ}\right) \cdot Q_{2}=-\dot{Q}_{2}^{T} Q_{2}+\dot{L}_{2} L_{2}^{-1} .
\end{aligned}
$$

But from the invariance property of $I_{r k}$ and the shape of $Q_{i}(t), i=1,2$, we have $Q_{1}^{T} D_{1} I_{r k}\left(g_{1}^{\circ}, g_{2}^{\circ}\right) Q_{1}=D_{1} I_{r k}\left(g_{1}, g_{2}\right)$ and $Q_{2}^{T} D_{1}^{\prime} I_{r k}\left(g_{1}^{\circ}, g_{2}^{\circ}\right) Q_{2}=D_{1}^{\prime} I_{r k}\left(g_{1}, g_{2}\right)$. The required relations then follow from $(*)$ by taking $\Pi_{\mathfrak{k}}$ of both sides.

In a similar way, we obtain

Theorem 6.7. Let $\left(g_{1}^{\circ}, g_{2}^{\circ}\right) \in G^{2}$. If $Q_{i}(t), L_{i}(t), i=1,2$, are the solutions of the factorization problems

$$
e^{-t D_{1} J_{r 0}\left(g_{1}^{\circ}, g_{2}^{\circ}\right)}=Q_{1}(t) L_{1}(t), \quad e^{-t D_{1}^{\prime} J_{r 0}\left(g_{1}^{\circ}, g_{2}^{\circ}\right)}=Q_{2}(t) L_{2}(t),
$$

where $Q_{i}(t) \in K, L_{i}(t) \in L$, then the $J_{r 0}$ flow defined by (6.3) and initial conditions $g_{1}(0)=g_{1}^{\circ}, g_{2}(0)=g_{2}^{\circ}$ is given by

$$
\begin{aligned}
& g_{1}(t)=Q_{1}^{T}(t) g_{1}^{\circ} Q_{2}(t)=L_{1}(t) g_{1}^{\circ} L_{2}(t)^{-1}, \\
& g_{2}(t)=Q_{2}^{T}(t) g_{2}^{\circ} Q_{1}(t)=L_{2}(t) g_{2}^{\circ} L_{1}(t)^{-1} .
\end{aligned}
$$

Theorem 6.9. Let $\left(g_{1}^{\circ}, g_{2}^{\circ}\right) \in G^{2}$. If $Z_{i}(t), X_{i}(t), i=1,2$, are the solutions of the factorization problems

$$
e^{-\frac{1}{2} D_{2} J_{r r}\left(g_{1}^{\circ}, g_{2}^{\circ}\right)}=Z_{1}(t) X_{1}(t), \quad e^{-\frac{1}{2} D_{2}^{\prime} J_{r r}\left(g_{1}^{\circ}, g_{2}^{\circ}\right)}=Z_{2}(t) X_{2}(t),
$$

where $Z_{i}(t) \in K, X_{i}(t) \in L$, then the $J_{r r}$ flow defined by (6.4) and initial conditions $g_{1}(0)=g_{1}^{\circ}, g_{2}(0)=g_{2}^{\circ}$ is given by

$$
g_{1}(t)=X_{2}(t) g_{1}^{\circ} X_{1}(t)^{-1}, \quad g_{2}(t)=Z_{1}^{T}(t) g_{2}^{\circ} Z_{2}(t)=X_{1}(t) g_{2}^{\circ} X_{2}(t)^{-1} .
$$

The rest of the section will be devoted to the $J_{r k}$ flows for $1 \leq k \leq r-1,2 \leq$ $r \leq n-1$. In order to obtain the Lax equation for $M_{h}\left(g_{1}, g_{2}\right)$ when $g_{1}, g_{2}$ evolve according to (6.2), we need some preparatory lemmas. 


\section{Lemma 6.11.}

$$
g_{2} D_{1} J_{r k}\left(g_{1}, g_{2}\right) g_{2}^{T}=D_{1}^{\prime} J_{r k}\left(g_{1}, g_{2}\right) g_{2} g_{2}^{T}+\left[g_{2} g_{2}^{T}, \nabla_{1}^{T} J_{r, k-1}\left(g_{1}, g_{2}\right) g_{2}^{T}\right] .
$$

Proof. Denote by $E_{j}(M)$ the $j$-th elementary symmetric function of the matrix $M$. From the formula

$$
\operatorname{det} M_{h, z}\left(g_{1}, g_{2}\right)=\sum_{r=0}^{n} \sum_{k=0}^{r} J_{r k}\left(g_{1}, g_{2}\right) h^{k} z^{n-r},
$$

we have

$$
E_{r}\left(M_{h}\right)=\sum_{k=0}^{r} J_{r k}\left(g_{1}, g_{2}\right) h^{k} .
$$

Differentiation with respect to $g_{1}$ yields

$$
\sum_{k} \nabla_{1}^{T} J_{r k}\left(g_{1}, g_{2}\right) h^{k}=\nabla^{T} E_{r}\left(M_{h}\right) g_{2}=-\sum_{j=0}^{r-1} E_{j}\left(M_{h}\right) M_{h}^{r-j-1} g_{2},
$$

which implies $\left[M_{h}, \sum_{k=0}^{r} \nabla_{1} J_{r k}\left(g_{1}, g_{2}\right) g_{2}^{-1} h^{k}\right]=0$. Now equate the coefficients of the powers of $h$ in the above expression to zero; we obtain $\left[g_{2} g_{1}, \nabla_{1}^{T} J_{r k} g_{2}^{-1}\right]=$ $\left[g_{2} g_{2}^{T}, \nabla_{1}^{T} J_{r, k-1} g_{2}^{-1}\right]$, which in turn implies the assertion.

\section{Lemma 6.12.}

$$
\begin{aligned}
& {\left[g_{2} g_{1}, D_{1}^{\prime} J_{r, k+1}\left(g_{1}, g_{2}\right)+D_{2} J_{r, k+1}\left(g_{1}, g_{2}\right)+g_{2} \nabla_{1} J_{r k}\left(g_{1}, g_{2}\right)-\nabla_{1}^{T} J_{r k}\left(g_{1}, g_{2}\right) g_{2}^{T}\right] } \\
= & {\left[g_{2} g_{2}^{T}, D_{1}^{\prime} J_{r k}\left(g_{1}, g_{2}\right)+D_{2} J_{r k}\left(g_{1}, g_{2}\right)+g_{2} \nabla_{1} J_{r, k-1}\left(g_{1}, g_{2}\right)-\nabla_{1}^{T} J_{r, k-1}\left(g_{1}, g_{2}\right) g_{2}^{T}\right] . }
\end{aligned}
$$

Proof. From $E_{r}\left(M_{h}\right)=\sum_{k=0}^{r} J_{r k}\left(g_{1}, g_{2}\right) h^{k}$, we obtain

$$
\sum_{k=0}^{r} D_{1}^{\prime} J_{r k}\left(g_{1}, g_{2}\right) h^{k}=\nabla^{T} E_{r}\left(M_{h}\right) g_{2} g_{1}
$$

and

$$
\sum_{k=0}^{r} D_{2} J_{r k}\left(g_{1}, g_{2}\right) h^{k}=M_{h} \nabla^{T} E_{r}\left(M_{h}\right)-h g_{2} g_{2}^{T} \nabla E_{r}\left(M_{h}\right) .
$$

Also, we have

$$
\sum_{k=1}^{r+1} g_{2} \nabla_{1} J_{r, k-1}\left(g_{1}, g_{2}\right) h^{k}=h g_{2} g_{2}^{T} \nabla E_{r}\left(M_{h}\right) .
$$

From these relations, it is easy to deduce that

$$
\begin{aligned}
& \sum_{k=1}^{r} B_{r k}\left(g_{1}, g_{2}\right) h^{k}+2 D_{1}^{\prime} J_{r 0}\left(g_{1}, g_{2}\right) \\
& \quad=2 M_{h} \nabla^{T} E_{r}\left(M_{h}\right)=-2 \sum_{j=1}^{r-1} E_{j}\left(M_{h}\right) M_{h}^{r-j}
\end{aligned}
$$

where

$$
\begin{aligned}
B_{r k}\left(g_{1}, g_{2}\right)= & D_{1}^{\prime} J_{r k}\left(g_{1}, g_{2}\right)+D_{2} J_{r k}\left(g_{1}, g_{2}\right)+g_{2} \nabla_{1} J_{r, k-1}\left(g_{1}, g_{2}\right) \\
& -\nabla_{1}^{T} J_{r, k-1}\left(g_{1}, g_{2}\right) g_{2}^{T} .
\end{aligned}
$$


Thus it follows from $(*)$ that

$$
\begin{aligned}
0= & {\left[M_{h}, \sum_{k=1}^{r} B_{r k}\left(g_{1}, g_{2}\right) h^{k}+2 D_{1}^{\prime} J_{r 0}\left(g_{1}, g_{2}\right)\right] } \\
= & \left(\left[g_{2} g_{1}, B_{r 1}\left(g_{1}, g_{2}\right)\right]-\left[g_{2} g_{2}^{T}, 2 D_{1}^{\prime} J_{r 0}\left(g_{1}, g_{2}\right)\right]\right) h \\
& +\sum_{k=2}^{r}\left(\left[g_{2} g_{1}, B_{r k}\left(g_{1}, g_{2}\right)\right]-\left[g_{2} g_{2}^{T}, B_{r, k-1}\left(g_{1}, g_{2}\right)\right]\right) h^{k} \\
& -\left[g_{2} g_{2}^{T}, B_{r, r}\left(g_{1}, g_{2}\right)\right] h^{r+1}
\end{aligned}
$$

We now introduce the Lie algebra $g \ell_{n}\left(\mathbf{C}\left[h, h^{-1}\right]\right)$ of $n \times n$ matrices with entries in $\mathbf{C}\left[h, h^{-1}\right]$. Then $g \ell_{n}\left(\mathbf{C}\left[h, h^{-1}\right]\right)$ contains two distinguished subalgebras,

$$
\tilde{\mathfrak{l}}=\left\{X(h)=\sum_{j \geq 0} X_{j} h^{j} \in g \ell_{n}(\mathbf{C}[h]) \mid X_{0} \in \mathfrak{l}\right\}
$$

and

$$
\tilde{\mathfrak{k}}=\left\{X(h)=\sum_{j \leq 0} X_{j} h^{j} \in g \ell_{n}\left(\mathbf{C}\left[h^{-1}\right]\right) \mid X_{0} \in \mathfrak{k}\right\} .
$$

Clearly, we have the direct sum decomposition

$$
g \ell_{n}\left(\mathbf{C}\left[h, h^{-1}\right]\right)=\tilde{\mathfrak{l}} \oplus \tilde{\mathfrak{k}} .
$$

We shall denote by $\Pi_{\tilde{\mathfrak{l}}}$ and $\Pi_{\tilde{\mathfrak{k}}}$ the associated projections.

Proposition 6.14. If $\left(g_{1}, g_{2}\right)$ evolve according to (6.2), then

$$
\dot{M}_{h}=\left[M_{h}, \Pi_{\tilde{\mathfrak{r}}}\left(h^{-k} M_{h} \nabla^{T} E_{r}\left(M_{h}\right)\right)\right]=\left[\Pi_{\tilde{\mathfrak{k}}}\left(h^{-k} M_{h} \nabla^{T} E_{r}\left(M_{h}\right)\right), M_{h}\right]
$$

Proof. From (6.2), we obtain

$$
\begin{aligned}
\dot{M}_{h}= & \frac{1}{2}\left[M_{h}, \Pi_{\tilde{\mathfrak{l}}}\left(D_{1}^{\prime} J_{r k}+D_{2} J_{r k}\right)\right]-\frac{h}{2} g_{2}\left(D_{1} J_{r k}+D_{1}^{T} J_{r k}\right) g_{2}^{T} \\
& -\frac{h}{2}\left(D_{2} J_{r k}\right) g_{2} g_{2}^{T}+\frac{h}{2} g_{2} g_{2}^{T}\left(D_{2} J_{r k}+D_{1}^{\prime} J_{r k}+\left(D_{1}^{\prime} J_{r k}\right)^{T}\right) .
\end{aligned}
$$

In order to cast this into the desired form, first note that by Lemma 6.11 ,

$$
\begin{aligned}
g_{2}\left(D_{1} J_{r k}+D_{1}^{T} J_{r k}\right) g_{2}^{T}= & \left(D_{1}^{\prime} J_{r k}\right) g_{2} g_{2}^{T}+g_{2} g_{2}^{T}\left(D_{1}^{\prime} J_{r k}\right)^{T} \\
& +\left[g_{2} g_{2}^{T}, \nabla_{1}^{T} J_{r, k-1} g_{2}^{T}-g_{2} \nabla_{1} J_{r, k-1}\right] .
\end{aligned}
$$

Substitute into the expression for $\dot{M}_{h}$ above and simplify; we obtain

$$
\begin{aligned}
\dot{M}_{h}= & \frac{1}{2}\left[M_{h}, \Pi_{\tilde{\mathfrak{l}}}\left(D_{1}^{\prime} J_{r k}+D_{2} J_{r k}\right)\right] \\
& -\frac{h}{2}\left[g_{2} g_{2}^{T}, D_{2} J_{r k}+D_{1}^{\prime} J_{r k}-\nabla_{1}^{T} J_{r, k-1} g_{2}^{T}+g_{2} \nabla_{1} J_{r, k-1}\right] .
\end{aligned}
$$

Now, we apply Lemma 6.12; after some algebra, we find that

$$
\begin{aligned}
& \frac{h}{2}\left[g_{2} g_{2}^{T}, D_{2} J_{r k}+D_{1}^{\prime} J_{r k}-\nabla_{1}^{T} J_{r, k-1} g_{2}^{T}+g_{2} \nabla_{1} J_{r, k-1}\right] \\
& \quad=\frac{1}{2}\left[M_{h}, \sum_{j=1}^{r-k}\left(D_{2} J_{r, k+j}+D_{1}^{\prime} J_{r, k+j}+g_{2} \nabla_{1} J_{r, k+j-1}-\nabla_{1}^{T} J_{r, k+j-1} g_{2}^{T}\right) h^{j}\right] .
\end{aligned}
$$


Finally, we substitute the above expression into $(\dagger)$ and make use of the relation $(*)$ in the proof of Lemma 6.12 ; this results in

$$
\dot{M}_{h}=\left[M_{h}, \Pi_{\tilde{\mathfrak{r}}}\left(h^{-k} M_{h} \nabla^{T} E_{r}\left(M_{h}\right)\right)\right] .
$$

In order to understand how to solve (6.15), define the $r$-matrix

$$
\tilde{R}=\Pi_{\tilde{\mathfrak{k}}}-\Pi_{\tilde{\mathfrak{l}}}
$$

associated with the splitting (6.13). We shall equip $g \ell_{n}\left(\mathbf{C}\left[h, h^{-1}\right]\right)$ with the adinvariant, nondegenerate pairing

$$
(X, Y)=\frac{1}{2 \pi \sqrt{-1}} \oint_{|h|=1} \operatorname{tr}(X(h) Y(h)) \frac{d h}{h}, \quad X, Y \in g \ell_{n}\left(\mathbf{C}\left[h, h^{-1}\right]\right) ;
$$

then the dual of $\tilde{R}$ with respect to the above pairing is given by

$$
\left(\tilde{R}^{*} X\right)(h)=\sum_{i<0} X_{i} h^{i}+R^{*} X_{0}-\sum_{i>0} X_{i} h^{i}, X(h)=\sum_{i} X_{i} h^{i} .
$$

Now, define

$$
\tilde{A}=\frac{1}{2}\left(\tilde{R}-\tilde{R}^{*}\right), S=\frac{1}{2}\left(\tilde{R}+\tilde{R}^{*}\right) .
$$

From (6.16) and (6.18), it is easy to show that

$$
(\tilde{A} X)(h)=\sum_{i<0} X_{i} h^{i}+A\left(X_{0}\right)-\sum_{i>0} X_{i} h^{i}, X(h)=\sum_{i} X_{i} h^{i} .
$$

As $A$ is a solution of $(m Y B)$, it follows from (6.20) that $\tilde{A}$ is also a solution of $(m Y B)$, so that $\tilde{R}$ is a classical $r$-matrix which satisfies hypothesis $(H)$ of [LP]. Consequently, we can equip the associative algebra $g \ell_{n}\left(\mathbf{C}\left[h, h^{-1}\right]\right)$ with the Poisson structure $[\mathrm{LP}]$

$$
\begin{aligned}
\{F, H\}(X)= & \frac{1}{2}(\tilde{A}(d F(X) X), d H(X) X)-\frac{1}{2}(\tilde{A}(X d F(X)), X d H(X)) \\
& +\frac{1}{2}(\tilde{S}(X d F(X)), d H(X) X)-\frac{1}{2}(\tilde{S}(d F(X) X), X d H(X)) .
\end{aligned}
$$

Consider the Hamiltonian

$$
H_{r k}(x)=\frac{1}{2 \pi \sqrt{-1}} \oint_{|h|=1} E_{r}(X(h)) \frac{d h}{h^{k+1}} .
$$

Differentiation gives

$$
\left(d H_{r k}(X)\right)(h)=h^{-k} \nabla^{T} E_{r}(X(h)),
$$

which shows $H_{r k}$ is an ad-invariant function. Hence the Hamilton equation generated by $H_{r k}$ in the Poisson structure in (6.21) is given by

$$
\dot{X}=\left[X, \Pi_{\mathfrak{l}}\left(h^{-k} X(h) \nabla^{T} E_{r}(X(h))\right)\right], \quad X \in g \ell_{n}\left(\mathbf{C}\left[h, h^{-1}\right]\right) .
$$

Clearly, equation (6.15) is a special case of (6.24), but more is true. Indeed, we can show that the finite dimensional subspace

$$
\tilde{\mathfrak{g}}_{2}=\left\{X_{0}+h X_{1} \mid X_{0}, X_{1} \in g \ell(n, \mathbf{C})\right\}
$$

is a Poisson submanifold of $g \ell_{n}\left(\mathbf{C}\left[h, h^{-1}\right]\right)$ equipped with the structure in (6.21). Therefore, the equation

$$
\left(X_{0}+h X_{1}\right)^{\cdot}=\left[X_{0}+h X_{1}, \Pi_{\mathfrak{l}}\left(h^{-k}\left(X_{0}+h X_{1}\right) \nabla^{T} E_{r}\left(X_{0}+h X_{1}\right)\right)\right]
$$


is a Hamiltonian system. Furthermore, the proof of Proposition 6.14 shows that if we solve (6.25) with initial conditions of the form $X(h, 0)=g_{2}^{\circ} g_{1}^{\circ}-h g_{2}^{\circ} g_{2}^{\circ T}$, then $X(h, t)$ is of the form $g_{2}(t) g_{1}(t)-h g_{2}(t) g_{2}^{T}(t)$.

Theorem 6.26 (Solution by factorization). For some $0<T \leq \infty$, there exist unique holomorphic matrix-valued functions

$$
\begin{aligned}
g_{+}(\cdot, t): \mathbf{C P}^{1} \backslash\{\infty\} & \rightarrow G L(n, \mathbf{C}), \\
g_{-}(\cdot, t): \mathbf{C P}^{1} \backslash\{0\} & \rightarrow G L(n, \mathbf{C}), \quad 0 \leq t<T,
\end{aligned}
$$

such that for $0 \leq t<T, g_{ \pm}(\cdot, t)$ are smooth in $t$, solve the factorization problem

$$
\exp \left\{t h^{-k} M_{h}\left(g_{1}^{\circ}, g_{2}^{\circ}\right) \nabla^{T} E_{r}\left(M_{h}\left(g_{1}^{\circ}, g_{2}^{\circ}\right)\right)\right\}=g_{+}(h, t) g_{-}(h, t)^{-1}, h \in \mathbf{C P}^{1} \backslash\{0, \infty\}
$$

and satisfy

$$
\begin{aligned}
& g_{+}(0, t) \in L, g_{+}(h, t)^{-1} \dot{g}_{+}(h, t) \in \operatorname{Im} \Pi_{\mathfrak{r}}, h \in \mathbf{C P}^{1} \backslash\{\infty\}, \\
& g_{-}(\infty, t) \in K, g_{-}(h, t)^{-1} \dot{g}_{-}(h, t) \in \operatorname{Im} \Pi_{\tilde{\mathfrak{k}}}, h \in \mathbf{C P}^{1} \backslash\{0\} .
\end{aligned}
$$

Moreover, for $0 \leq t<T, M_{h}(t)=g_{ \pm}^{-1}(h, t) M_{h}\left(g_{1}^{\circ}, g_{2}^{\circ}\right) g_{ \pm}(h, t)$ solves equation (6.25) with initial data $M_{h}(0)=g_{2}^{\circ} g_{1}^{\circ}-h g_{2}^{\circ}, g_{2}^{\circ T}$. Finally, if $\left(g_{1}^{\circ}, g_{2}^{\circ}\right) \in \mathcal{U}$ satisfies (GA1) and (GA2), $g_{ \pm}(h, t)$ can be constructed by means of theta functions associated with the spectral curve $\mathcal{C}\left(g_{1}^{\circ}, g_{2}^{\circ}\right)$.

Remark 6.30. We are unable to settle the question of whether equation (6.2) has a global solution when $1 \leq k \leq r-1,2 \leq r \leq n-1$. Hence we do not know whether $T$ is finite or infinite in the statement of the above theorem.

Proof of Theorem 6.26. First, the uniqueness of $g_{ \pm}$is clear. Now, let $g_{1}(t), g_{2}(t)$ denote the solution to equation (6.2) with $g_{1}(0)=g_{1}^{0}, g_{2}(0)=g_{2}^{0}$, valid for $0 \leq t<$ $T$. To prove existence of $g_{ \pm}$, we simply solve the linear equations

$$
\begin{aligned}
& \dot{g}_{+}(h, t)=g_{+}(h, t) \Pi_{\tilde{\mathfrak{l}}}\left(h^{-k} M_{h}\left(g_{1}(t), g_{2}(t)\right) \nabla^{T} E_{r}\left(M_{h}\left(g_{1}(t), g_{2}(t)\right)\right)\right), \\
& \dot{g}_{-}(h, t)=-g_{-}(h, t) \Pi_{\tilde{\mathfrak{k}}}\left(h^{-k} M_{h}\left(g_{1}(t), g_{2}(t)\right) \nabla^{T} E_{r}\left(M_{h}\left(g_{1}(t), g_{2}(t)\right)\right)\right),
\end{aligned}
$$

with initial conditions $g_{ \pm}(h, 0)=I$. Then the properties (6.28) and (6.29) are immediate. The analyticity properties are also clear by standard arguments. To show that $M_{h}(t)$ solves equation (6.25), differentiate the product $g_{+}(h, t) g_{-}(h, t)^{-1}$. This gives

$$
\begin{aligned}
\frac{d}{d t}\left(g_{+}(h, t) g_{-}(h, t)^{-1}\right) \\
=h^{-k}\left\{g _ { + } ( h , t ) M _ { h } ( g _ { 1 } ( t ) , g _ { 2 } ( t ) ) g _ { + } ( h , t ) ^ { - 1 } \nabla ^ { T } E _ { r } \left(g_{+}(h, t)\right.\right. \\
\left.\left.\cdot M_{h}\left(g_{1}(t), g_{2}(t)\right) g_{+}(h, t)^{-1}\right)\right\} g_{+}(h, t) g_{-}(h, t)^{-1} .
\end{aligned}
$$

But by Proposition 6.14,

$$
\frac{d}{d t}\left(g_{+}(h, t) M_{h}\left(g_{1}(t), g_{2}(t)\right) g_{+}(h, t)^{-1}\right)=0 .
$$

This implies $g_{+}(h, t) M_{h}\left(g_{1}(t), g_{2}(t)\right) g_{+}(h, t)^{-1}=M_{h}\left(g_{1}^{\circ}, g_{2}^{\circ}\right)$, and hence

$$
\begin{aligned}
& \frac{d}{d t}\left(g_{+}(h, t) g_{-}(h, t)^{-1}\right) \\
& \quad=\left\{h^{-k} M_{h}\left(g_{1}^{\circ}, g_{2}^{\circ}\right) \nabla^{T} E_{r}\left(M_{h}\left(g_{1}^{\circ}, g_{2}^{\circ}\right)\right)\right\} g_{+}(h, t) g_{-}(h, t)^{-1} .
\end{aligned}
$$


Therefore,

$$
g_{+}(h, t) g_{-}(h, t)^{-1}=\exp \left\{h^{-k} M_{h}\left(g_{1}^{\circ}, g_{2}^{\circ}\right) \nabla^{T} E_{r}\left(M_{h}\left(g_{1}^{\circ}, g_{2}^{\circ}\right)\right)\right\} .
$$

The construction of $g_{ \pm}$will be dealt with in what follows.

In the remainder of the section, we assume that $\left(g_{1}^{\circ}, g_{2}^{\circ}\right) \in \mathcal{U}$ satisfies (GA1) and (GA2). The goal is to construct $g_{ \pm}$in terms of theta functions associated with the spectral curve $\mathcal{C}\left(g_{1}^{\circ}, g_{2}^{\circ}\right)$ and to recover $g_{1}(t), g_{2}(t), 0<t<T$. Thus, all calculations and arguments in what follows are for $t \in(0, T)$ without further mention. We begin by considering the initial data $\left(g_{1}^{\circ}, g_{2}^{\circ}\right)$ and the associated matrix pencil $M_{h}\left(g_{1}^{\circ}, g_{2}^{\circ}\right)=g_{2}^{\circ} g_{1}^{\circ}-h g_{2}^{\circ} g_{2}^{\circ T}$. Let $0<\mu_{1}<\cdots<\mu_{n}$ be the eigenvalues of $g_{1}^{\circ}, g_{2}^{\circ T}$ and set $\mu=\operatorname{diag}\left(\mu_{1}, \ldots, \mu_{n}\right)$. Since $g_{2}^{\circ} g_{2}^{\circ T}$ is symmetric, there exists $O_{0} \in K$ such that $g_{2}^{\circ} g_{2}^{\circ T}=O_{0} \mu O_{0}^{T}$. Clearly,

$$
M_{h}\left(g_{1}^{\circ}, g_{2}^{\circ}\right)=O_{0} M_{h}\left(O_{0}^{T} g_{1}^{\circ} O_{0}, O_{0}^{T} g_{2}^{\circ} O_{0}\right) O_{0}^{T}
$$

and

$$
M_{h}\left(O_{0}^{T} g_{1}^{\circ} O_{0}, O_{0}^{T} g_{2}^{\circ} O_{0}\right)=O_{0}^{T} g_{2}^{\circ} g_{1}^{\circ} O_{0}-h \mu .
$$

Let $O_{0}^{T} g_{2}^{\circ} g_{1}^{\circ} O_{0} u-h \mu-z I$ be denoted by $M\left(O_{0}^{T} g_{1}^{\circ} O_{0}, O_{0}^{T} g_{2}^{\circ} O_{0} ; p\right), p=[u: h: z] \in$ $\mathcal{C}\left(g_{1}^{\circ}, g_{2}^{\circ}\right)$. There exists a unique $\widehat{v}(p) \in \operatorname{ker} M\left(O_{0}^{T} g_{1}^{\circ} O_{0}, O_{0}^{T} g_{2}^{\circ} O_{0} ; p\right)$ with $\left(\widehat{v}(p), e_{1}\right)$ $=1$. Furthermore,

$$
\left(\widehat{v}_{j}\right) \geq-D^{\prime}(0)+P_{-}^{1}-P_{-}^{j}
$$

by Corollary 5.10, where $D^{\prime}(0)$ is an effective divisor of degree $g_{c}=\frac{1}{2}(n-1)(n-2)$ and $D^{\prime}(0)-P_{-}^{1}+P_{-}^{j}$ is regular for each $j$. Now we put in the dynamics. We have

$$
g_{2}(t) g_{2}^{T}(t)=O(t) \mu O^{T}(t), \quad O(0)=O_{0},
$$

and a direct calculation shows that

$$
\dot{O}=-\frac{1}{2}\left(-\Pi_{\mathfrak{k}}\left(D_{1}^{\prime} J_{r k}+D_{2} J_{r k}\right)+\nabla_{1}^{T} J_{r, k-1} g_{2}^{T}-g_{2} \nabla_{1} J_{r, k-1}\right) O .
$$

Using (6.35), Proposition 6.14 and the invariance property of $J_{r k}$, the evolution of $M_{h}\left(O^{T} g_{1} O, O^{T} g_{2} O\right)=O^{T} g_{2} g_{1} O-h \mu$ is given by

$$
\begin{aligned}
& \left(M_{h}\left(O^{T} g_{1} O, O^{T} g_{2} O\right)\right)^{\cdot} \\
& \quad=\left[M_{h}\left(O^{T} g_{1} O, O^{T} g_{2} O\right),\right. \\
& \left.\quad\left(h^{-k} M_{h}\left(O^{T} g_{1} O, O^{T} g_{2} O\right) \nabla^{T} E_{r}\left(M_{h}\left(O^{T} g_{1} O, O^{T} g_{2} O\right)\right)\right)_{+}\right],
\end{aligned}
$$

where $(\quad)_{+}$is the projection onto $g \ell_{n}(\mathbf{C}[h])$. Let

$$
\tilde{g}_{ \pm}(h, t)=O_{0}^{T} g_{ \pm}(h, t) O(t)
$$

then the solution to (6.36) takes the form

$$
M_{h}\left(O^{T}(t) g_{1}(t) O(t), O^{T}(t) g_{2}(t) O(t)\right)=\tilde{g}_{ \pm}(h, t)^{-1} M_{h}\left(O_{0}^{T} g_{1}^{\circ} O_{0}, O_{0}^{T} g_{2}^{\circ} O_{0}\right) \tilde{g}_{ \pm}(h, t)
$$

and $\tilde{g}_{ \pm}$solves the factorization problem

$$
\begin{gathered}
\exp \left\{t h^{-k} M_{h}\left(O_{0}^{T} g_{1}^{\circ} O_{0}, O_{0}^{T} g_{2}^{\circ} O_{0}\right) \nabla^{T} E_{r}\left(M_{h}\left(O_{0}^{T} g_{1}^{\circ} O_{0}, O_{0}^{T} g_{2}^{\circ} O_{0}\right)\right)\right\} \\
=\tilde{g}_{+}(h, t) \tilde{g}_{-}(h, t)^{-1}, \quad h \in \mathbf{C P}^{1} \backslash\{0, \infty\} .
\end{gathered}
$$


From the definition of $E_{r}$, we clearly have

$$
\begin{gathered}
h(p)^{-k} M_{h(p)}\left(O_{0}^{T} g_{1}^{\circ} O_{0}, O_{0}^{T} g_{2}^{\circ} O_{0}\right) \nabla^{T} E_{r}\left(M_{h(p)}\left(O_{0}^{T} g_{1}^{\circ} O_{0}, O_{0}^{T} g_{2}^{\circ} O_{0}\right)\right) \widehat{v}(p) \\
=\mu_{r k}(p) \widehat{v}(p),
\end{gathered}
$$

where $\mu_{r k}$ is meromorphic on $\mathcal{C}\left(g_{1}^{\circ}, g_{2}^{\circ}\right)$. Set

$$
v_{ \pm}(t, p)=\tilde{g}_{ \pm}(h(p), t)^{-1} \widehat{v}(p) .
$$

It follows from (6.39), (6.40) and (6.38) that

$$
\begin{gathered}
e^{t \mu_{r k}(p)} v_{+}(t, p)=v_{-}(t, p), h(p) \in \mathbf{C P}^{1} \backslash\{0, \infty\}, \\
M_{h(p)}\left(O^{T}(t) g_{1}(t) O(t), O^{T}(t) g_{2}(t) O(t)\right) v_{ \pm}(t, p)=z(p) v_{ \pm}(t, p) .
\end{gathered}
$$

Moreover, by $(6.38)$ and $(\mathrm{GA} 2)_{2}$ the matrix $\tilde{g}_{-}(\infty, t)$ must be diagonal, so that

$$
\begin{aligned}
& \left(v_{+}^{j}(t, \cdot)\right) \geq-D^{\prime}(0) \quad \text { on } \mathcal{C}\left(g_{1}^{\circ}, g_{2}^{\circ}\right) \backslash \operatorname{supp} P_{-}, \\
& \left(v_{-}^{j}(t, \cdot)\right) \geq-D^{\prime}(0)+P_{-}^{1}-P_{-}^{j} \text { on } \mathcal{C}\left(g_{1}^{\circ}, g_{2}^{\circ}\right) \backslash \operatorname{supp} P_{+} .
\end{aligned}
$$

In this way, we are led to scalar factorization problems which can be solved by using Baker-Akhiezer functions [RSTS]. To write down explicit formulas for the $v_{ \pm}^{j}$ 's, fix a canonical homology basis $\left\{a_{j}, b_{k}\right\}_{1 \leq j, k \leq g_{c}}$ of the Riemann surface associated with the smooth curve $\mathcal{C}\left(g_{1}^{\circ}, g_{2}^{\circ}\right)$, and let $\left\{\omega_{i}\right\}_{1 \leq i \leq g_{c}}$ be a cohomology basis dual to $\left\{a_{j}, b_{k}\right\}$, i.e. $\int_{a_{j}} \omega_{i}=\delta_{i j}, \int_{b_{j}} \omega_{i}=\Omega_{i j}$. With respect to the Riemann matrix $\Omega=\left(\Omega_{i j}\right)$, we construct the theta function

$$
\theta(\vec{z}) \equiv \theta(\vec{z}, \Omega)=\sum_{\vec{n} \in \mathbf{Z}^{g_{c}}} \exp \{2 \pi \sqrt{-1} \vec{n} \cdot \vec{z}+\pi \sqrt{-1} \vec{n} \cdot \Omega \vec{n}\}
$$

Choose a non-singular $\vec{e}$ in the theta divisor (i.e. $\theta(\vec{e})=0, E_{\vec{e}} \equiv \theta\left(\vec{e}+\int_{x}^{y} \vec{\omega}\right) \not \equiv$ $0)$ with the additional property that $E_{\vec{e}}\left(P_{-}^{j}, P\right) \not \equiv 0$ for each $j$. Let $P_{\infty}$ be a fixed point "at $\infty$ " on $\mathcal{C}\left(g_{1}^{\circ}, g_{2}^{\circ}\right)$. Then as $\theta(\vec{e})=0$, there exists $D_{g_{c}-1} \in$ $\operatorname{Div}^{g_{c}-1}\left(\mathcal{C}\left(g_{1}^{\circ}, g_{2}^{\circ}\right)\right)$ such that

$$
\vec{e}=\vec{\Delta}-\int_{\left(g_{c}-1\right) P_{\infty}}^{D_{g_{c}-1}} \vec{\omega} \quad([\mathrm{M}], \text { p. } 160),
$$

where $\vec{\Delta}$ is the vector of Riemann constants. Furthermore, as $D^{\prime}(0)$ is regular, we have

$$
\theta\left(\vec{e}+\int_{D^{\prime}(0)}^{D_{g_{c}-1}+P} \vec{\omega}\right)=0 \Leftrightarrow P \in D^{\prime}(0) .
$$

Now, let $\phi_{+}(p)$ be a meromorphic differential of the second kind with poles only at $\left\{P_{-}^{j}\right\}_{j=1}^{n}$ chosen such that

$$
\begin{gathered}
\phi_{-}(p) \equiv d \mu_{r k}(p)+\phi_{+}(p) \quad \text { is regular in } \mathcal{C}\left(g_{1}^{\circ}, g_{2}^{\circ}\right) \backslash \operatorname{supp} P_{+}, \\
\int_{a i} \phi_{+}=0, \quad i=1, \ldots, g_{c} .
\end{gathered}
$$


Then

$$
v_{ \pm}^{j}(t, p)=c_{j}(t) \exp \left(t \int_{P_{\infty}}^{p} \phi_{ \pm}\right) \frac{E_{\vec{e}}\left(P_{-}^{1}, p\right) \theta\left(\vec{e}+\int_{D^{\prime}(0)+P_{-}^{j}}^{D_{g_{c}-1}+P_{-}^{1}+p} \vec{\omega}+t \Phi_{+}\right)}{E_{\vec{e}}\left(p_{-}^{j}, p\right) \theta\left(\vec{e}+\int_{D^{\prime}(0)}^{D_{c_{c}-1}+p} \vec{\omega}\right)}
$$

where $\Phi_{+}$is the vector of $b_{i}$-periods and $c_{j}(t)$ are non-vanishing functions to be determined. Indeed, using the periodicity properties of $\theta$, it is easy to check that the right hand side of (6.46) is well defined and has the desired properties. In particular, when $t=0$, we obtain

$$
\hat{v}_{j}(p)=c_{j}(0) \frac{E_{\vec{e}}\left(P_{-}^{1}, p\right) \theta\left(\vec{e}+\int_{D^{\prime}(0)+P_{-}^{j}}^{D_{g_{c}-1}+P^{1}+p} \vec{\omega}\right)}{E_{\vec{e}}\left(P_{-}^{j}, p\right) \theta\left(\vec{e}+\int_{D^{\prime}(0)}^{D_{g_{-}-1}+p} \vec{\omega}\right)} .
$$

Clearly, $c_{1}(0)=1$; the other $c_{j}(0)$ 's are then uniquely determined from the definition of $\hat{v}(p)$. Let

$$
c(t)=\operatorname{diag}\left(c_{1}(t), \ldots, c_{n}(t)\right) .
$$

In view of (6.46), we shall write

$$
v_{ \pm}(t, p)=c(t) v_{ \pm}^{\theta}(t, p)
$$

where the $v_{ \pm}^{\theta}(t, p)$ are known. Given $h \in \mathbf{C}$ which is not a branch point of the coordinate function $h$, there exist $n$ points $P_{1}(h), \ldots, P_{n}(h)$ of $\mathcal{C}\left(g_{1}^{\circ}, g_{2}^{\circ}\right)$ lying over $h$. Hence we can define the matrices

$$
\begin{gathered}
\widehat{V}(h)=\left(\hat{v}\left(P_{1}(h)\right), \ldots, \hat{v}\left(P_{n}(h)\right)\right), V_{ \pm}(h, t)=\left(v_{ \pm}\left(t, P_{1}(h)\right), \ldots, v_{ \pm}\left(t, P_{n}(h)\right)\right), \\
V_{ \pm}^{\theta}(h, t)=\left(v_{ \pm}^{\theta}\left(t, P_{1}(h)\right), \ldots, v_{ \pm}^{\theta}\left(t, P_{n}(h)\right)\right) .
\end{gathered}
$$

Note that from (6.37), (6.41) and (6.49), we obtain the relations

$$
g_{ \pm}(h, t)=O_{0} \widehat{V}(h)\left(V_{ \pm}^{\theta}(h, t)\right)^{-1} c(t)^{-1} O^{T}(t),
$$

where $c(t)$ and $O(t)$ are as yet undetermined. Now, from (6.29), we have $g_{-}(\infty, t)=$ $\overline{g_{-}(\infty, t)}$ and $g_{-}(\infty, t) g_{-}^{T}(\infty, t)=I$. This implies

$$
|c(t)|^{2}=\left(\lim _{h \rightarrow \infty} \widehat{V}(h) V_{-}^{\theta}(h, t)^{-1}\right)^{*}\left(\lim _{h \rightarrow \infty} \widehat{V}(h) V_{-}^{\theta}(h, t)^{-1}\right) .
$$

So $|c(t)|$ is determined. Write $c(t)=|c(t)| \delta(t)$, where $\delta(t)$ is unitary diagonal. Then from

$$
O_{0} \widehat{V}(0)\left(V_{+}^{\theta}(0, t)\right)^{-1}|c(t)|^{-1}=g_{+}(0, t) O(t) \delta(t),
$$

we see that the right hand side gives the unique (lower) $\times$ (unitary) decomposition of the known left hand side. Consequently, $O(t) \delta(t)$ is now determined, and this in turn implies $g_{ \pm}(h, t)$ are determined as everything on the right hand side of $(6.51)$ is known. From Theorem 6.26, this means that we now have $g_{2}(t) g_{1}(t)-h g_{2}(t) g_{2}^{T}(t)$; so it remains to recover $g_{1}(t)$ and $g_{2}(t)$. In order to accomplish this, we need the information (obtained in section 4) that the evolution $\left(g_{1}(t), g_{2}(t)\right)$ is taking place on the level set of integrals $I^{c}\left(g_{1}^{\circ}, g_{2}^{\circ}\right)$, so that in particular

$$
\lambda_{r k}\left(g_{1}(t), g_{2}(t)\right)=\lambda_{r k}\left(g_{1}^{\circ}, g_{2}^{\circ}\right), \quad 1 \leq r \leq n-k, 1 \leq k \leq n-1,
$$




$$
\begin{aligned}
\operatorname{sgn} F_{k}\left(g_{1}(t), g_{2}(t)\right) & =\operatorname{sgn} F_{k}\left(g_{1}^{\circ}, g_{2}^{\circ}\right), \text { i.e. } \\
\operatorname{det}\left(g_{2}(t)\right)_{n-k} & =\operatorname{det}\left(g_{2}^{\circ}\right)_{n-k}, \quad k=1, \ldots, n .
\end{aligned}
$$

Let $g_{2}(t)=\ell(t) q(t)$, where $\ell(t) \in L, q(t) \in K$. Since $g_{2}(t) g_{2}^{T}(t)$ is known, we can determine $\ell(t)$ from the relation

$$
g_{2}(t) g_{2}^{T}(t)=\ell(t) \ell^{T}(t)
$$

As a result, $q(t) g_{1}(t)$ is also determined. Therefore, in order to get $g_{1}(t)$ and $g_{2}(t)$, all we need to do now is find $q(t)$. Set $q_{i}(t)=q(t) e_{i}, i=1, \ldots, n$, where $\left\{e_{i}\right\}_{1 \leq i \leq n}$ is the canonical basis of $\mathbf{R}^{n}$. The construction of $q(t)$ proceeds as follows. First, we construct $q_{n}(t)$, by requiring that

$$
\begin{gathered}
q_{n}(t) \perp\left(q(t) g_{1}(t)-\lambda_{r 1}\left(g_{1}^{\circ}, g_{2}^{\circ}\right) \ell(t)^{-1}\right)^{T} e_{1}, \quad 1 \leq r \leq n-1, \\
\left\|q_{n}(t)\right\|=1
\end{gathered}
$$

(note that

$$
\begin{aligned}
\left(q_{n}(t),\left(q(t) g_{1}(t)-\lambda \ell(t)^{-1}\right)^{-T} e_{1}\right) & =\left(e_{1},\left(g_{1}(t)-\lambda g_{2}(t)^{-1}\right)^{-1} e_{n}\right) \\
& =(-1)^{n+1} \frac{Q_{1}\left(g_{1}(t), g_{2}(t), \lambda\right)}{Q_{0}\left(g_{1}(t), g_{2}(t), \lambda\right)}
\end{aligned}
$$

so $(*)$ is just the definition of $\left.\lambda_{r 1}\left(g_{1}(t) g_{2}(t)\right), 1 \leq r \leq n-1\right)$. Clearly, $(*)$ is invariant under complex conjugation; hence there always exists a real, nonzero solution. Consequently $(*)$ has at least two real solutions $\pm q_{n}(t)$. Fixing $q_{n}(t)$, we obtain $q_{n-1}(t)$ by solving the system

$$
\begin{gathered}
q_{n-1}(t) \wedge q_{n}(t) \perp\left(\left(q(t) g_{1}(t)-\lambda_{r 2}\left(g_{1}^{\circ}, g_{2}^{\circ}\right) \ell(t)^{-1}\right)^{-T}\right)^{\wedge 2} e_{1} \wedge e_{2}, 1 \leq r \leq n-2, \\
\left\|q_{n-1}(t)\right\|=1
\end{gathered}
$$

(note that

$$
\begin{aligned}
& \left(q_{n-1}(t) \wedge q_{n}(t),\left(\left(q(t) g_{1}(t)-\lambda \ell(t)^{-1}\right)^{-T}\right)^{\wedge 2} e_{1} \wedge e_{2}\right) \\
& \quad=\left(e_{1} \wedge e_{2},\left(\left(g_{1}(t)-\lambda g_{2}(t)^{-1}\right)^{-1}\right)^{\wedge 2} e_{n-1} \wedge e_{n}\right)=(-1)^{2(n+1)} \frac{Q_{2}\left(g_{1}(t), g_{2}(t), \lambda\right)}{Q_{0}\left(g_{1}(t), g_{2}(t), \lambda\right)},
\end{aligned}
$$

so $(* *)$ is just the definition of $\left.\lambda_{r 2}\left(g_{1}(t), g_{2}(t)\right), 1 \leq r \leq n-2\right)$. Again, this has at least two real solutions $\pm q_{n-1}(t)$. By induction, we can construct at least $2^{n}$ real orthogonal matrices, and by invoking local uniqueness, we have exactly $2^{n}$ such matrices which differ from each other at most by a choice of signs $\pm q_{n}(t)$, $\pm q_{n-1}(t), \ldots, \pm q_{1}(t)$. But then the signs are fixed by using (6.55). Hence $q(t)$ is uniquely determined.

\section{REFERENCES}

[AL] Ablowitz, M., Ladik, J., Nonlinear differential-difference equations and Fourier analysis, J. Math. Phys. 17 (1976), 1011-1018. MR 55:897

[AvM] Adler, M., van Moerbeke, P., Linearization of Hamiltonian systems, Jacobi varieties and representation theory, Adv. Math. 38 (1980), 318-379. MR 83m:58042

[C] Chu, M., A continuous approximation to the generalized Schur decomposition, Linear Alg. Appl. 78 (1986), 119-132. MR 87i:15006

[D] Drinfeld, V., Hamiltonian structures on Lie groups, Lie bialgebras and the geometric meaning of the classical Yang-Baxter equations, Sov. Math. Dokl. 27 (1983), 68-71. MR 84i:58044 (Russian original) 
[DKN] Dubrovin, B., Krichever, I., Novikov, S., Integrable systems, I, In: Arnold, V., Novikov, S. (eds):, Dynamical systems, IV, Springer-Verlag, Berlin, 1990. MR 87k:58112 (Russian original)

[DL] Deift, P., Li, L.C., Generalized affine Lie algebras and the solution of a class of flows associated with the QR eigenvalue algorithm, Comm. Pure Appl. Math. 42 (1989), 963991. MR 92a:58063

[DLT] Deift, P., Li, L.C., Tomei, C., Matrix factorizations and integrable systems, Comm. Pure Appl. Math. 42 (1989), 443-521. MR 92a:58062

[DLNT] Deift, P., Li, L.C., Nanda, T., Tomei, C., The Toda flow on a generic orbit is integrable, Comm. Pure Appl. Math. 39 (1986), 183-232. MR 87c:58045

[DS] Drinfeld, V.G., Sokolov, V.V., Lie algebras and equations of Korteweg-de Vries type, J. Sov. Math. 30 (1985), 1975-2036. MR 86h:58071 (Russian original)

[F] Forster, O., Lectures on Riemann surfaces, Springer-Verlag, Berlin, 1981. MR 83d:30046

[FT] Faddeev, L., Takhtajan, L., Hamiltonian approach to soliton theory, Springer-Verlag, Berlin, 1987. MR 89m:58103

[G] Griffiths, P., Linearizing flows and a cohomological interpretation of Lax equations, Amer. J. Math. 107 (1985), 1445-1484. MR 87c:58048

[GD] Gelfand, I.M., Dikii, L., Fractional powers of operators and Hamiltonian systems, Funct. Anal. Appl. 10 (1976), 259-273. MR 55:6484 (Russian original)

[GG] Golubitsky, M., Guillemin, V., Stable mappings and their singularities, Springer-Verlag, Berlin, 1973. MR 49:6269

[L1] Li, L. C., On the complete integrability of some Lax systems on $G L(n, \mathbf{R}) \times G L(n, \mathbf{R})$, Bull. Amer. Math. Soc. (N.S.) 23 (1990), 487-493. MR 91a:58082

[L2] - Two results on a class of Poisson structures on Lie groups, Math. Z. 210 (1992), 327-338. MR 94d:58064

[L3] , The SVD flows on generic symplectic leaves are completely integrable, to appear in Adv. Math.

[LP] Li, L.C., Parmentier, S., A new class of quadratic Poisson structures and the YangBaxter equation, C.R. Acad. Sci. Paris Sér. I Math. 307 (1988), 279-281; Nonlinear Poisson structures and r-matrices, Commun. Math. Phys. 125 (1989), 545-563. MR 90a:58072; MR 90i:58074

[M] Mumford, D., Tata lectures on theta, I, Birkhauser, Boston, 1983. MR 85h:14026

[MS] Moler, C.B., Stewart, G.W., An algorithm for generalized matrix eigenvalue problems, SIAM J. Num. Anal. 10 (1973), 241-256. MR 49:10135

[R1] Rosenlicht, M., Equivalence relations on algebraic curves, Ann. of Math. 56 (1952), 169-191. MR 14:80c

[R2] Generalized Jacobian varieties, Ann. of Math. 59 (1954), 505-530. MR 15:823b

[RSTS] Reyman, A.G., Semenov-Tian-Shansky, M., Reduction of Hamiltonian systems affine Lie algebras and Lax equations, II, Invent. Math. 63 (1981), 423-432. MR 82k:58049

[S] Serre, J.-P, Algebraic groups and class fields, Springer-Verlag, Berlin, 1988. MR 88i: 14041

[STS1] Semenov-Tian-Shansky, M., What is a classical r-matrix?, Funct. Anal. Appl. 17 (1983), 259-272. MR 85i:58071 (Russian original)

[STS2] - Dressing transformations and Poisson group actions, Publ. Res. Inst. Math. Sci. 21 (1985), 1237-1260. MR 88b:58057

[V] Vinnikov, V., Complete description of determinental representations of smooth irreducible curves, Linear Alg. Appl. 125 (1989), 103-140. MR 90m:14028

[VdW] van der Waerden, B.L., Algebra, Vol. 2, Ungar, New York, 1970. MR 41:8187b

[W] Weinstein, A., The local structure of Poisson manifolds, J. Diff. Geom. 18 (1983), 523557; 22 (1985), 255. MR 86i:58059; MR 87k:58099

Department of Mathematics, Pennsylvania State University, University Park, PennSYLVANIA 16802

E-mail address: luenli@math.psu.edu 